\title{
Search for the direct production of charginos, neutralinos and staus in final states with at least two hadronically decaying taus and missing transverse momentum in $p p$ collisions at $\sqrt{s}=8 \mathrm{TeV}$ with the ATLAS detector
}

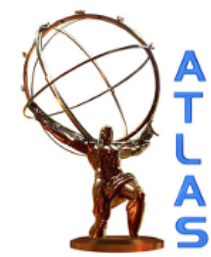

\section{The ATLAS collaboration}

E-mail: atlas.publications@cern.ch

ABSTRACT: Results of a search for the electroweak associated production of charginos and next-to-lightest neutralinos, pairs of charginos or pairs of tau sleptons are presented. These processes are characterised by final states with at least two hadronically decaying tau leptons, missing transverse momentum and low jet activity. The analysis is based on an integrated luminosity of $20.3 \mathrm{fb}^{-1}$ of proton-proton collisions at $\sqrt{s}=8 \mathrm{TeV}$ recorded with the ATLAS experiment at the Large Hadron Collider. No significant excess is observed with respect to the predictions from Standard Model processes. Limits are set at $95 \%$ confidence level on the masses of the lighter chargino and next-to-lightest neutralino for various hypotheses for the lightest neutralino mass in simplified models. In the scenario of direct production of chargino pairs, with each chargino decaying into the lightest neutralino via an intermediate tau slepton, chargino masses up to $345 \mathrm{GeV}$ are excluded for a massless lightest neutralino. For associated production of mass-degenerate charginos and next-tolightest neutralinos, both decaying into the lightest neutralino via an intermediate tau slepton, masses up to $410 \mathrm{GeV}$ are excluded for a massless lightest neutralino.

KeYwORDS: Hadron-Hadron Scattering

ARXIV EPRINT: 1407.0350 


\section{Contents}

1 Introduction 1

2 SUSY scenarios $\quad 2$

3 The ATLAS detector 3

4 Data sample 4

5 Monte Carlo simulation 4

5.1 Standard Model processes 4

5.2 SUSY processes 5

$\begin{array}{llr}6 & \text { Event reconstruction } & 6\end{array}$

$\begin{array}{lll}7 & \text { Event selection } & 9\end{array}$

8 Standard Model background estimation $\quad 11$

$\begin{array}{lll}8.1 & \text { Multi-jet background estimation } & 12\end{array}$

8.2 W+jets background estimation 14

$\begin{array}{lll}8.3 & \text { Estimation of irreducible backgrounds } & 17\end{array}$

$\begin{array}{ll}8.4 & \text { Fitting procedure }\end{array}$

9 Systematic uncertainties $\quad 20$

10 Results $\quad 22$

11 Interpretation $\quad \mathbf{2 3}$

11.1 Simplified models: chargino-neutralino and chargino-chargino production $\quad 24$

$\begin{array}{ll}11.2 \text { Direct stau production } & 26\end{array}$

$\begin{array}{ll}11.3 \text { The pMSSM model } & 26\end{array}$

12 Conclusion $\quad 28$

$\begin{array}{ll}\text { The ATLAS collaboration } & 36\end{array}$

\section{Introduction}

Supersymmetry (SUSY) [1-9] is a promising extension of the Standard Model (SM) of particle physics. For each SM particle supersymmetry predicts the existence of a super-partner (also referred to as a 'sparticle'), whose spin differs by one half unit from the corresponding 
SM partner. Supersymmetric theories provide elegant solutions to unanswered questions of the SM, such as the hierarchy problem [10-13]. In $R$-parity-conserving SUSY models [1418], SUSY particles are always produced in pairs and the lightest supersymmetric particle (LSP) provides a dark matter candidate [19-21].

In SUSY models, the mass eigenstates formed from the linear superpositions of the SUSY partners of the charged and neutral Higgs bosons and electroweak gauge bosons, the charginos $\left(\tilde{\chi}_{i}^{ \pm}, i=1,2\right)$ and neutralinos $\left(\tilde{\chi}_{j}^{0}, j=1,2,3,4\right.$ in the order of increasing masses), as well as the sleptons (superpartners of the leptons, ${ }^{1} \tilde{\ell}$ and $\tilde{\nu}$ ) can be sufficiently light to be produced at the Large Hadron Collider (LHC) [22]. Naturalness arguments suggest that the lightest third-generation sparticles, charginos and neutralinos should have masses of a few hundred $\mathrm{GeV}$ to protect the Higgs boson mass from quadratically divergent quantum corrections [23, 24]. Furthermore, light sleptons could play a role in the co-annihilation of neutralinos, leading to a dark matter relic density consistent with cosmological observations [25, 26], and their mass is expected to be in the $\mathcal{O}(100 \mathrm{GeV})$ range in gauge-mediated [27-32] and anomaly-mediated [33, 34] SUSY breaking scenarios. Models with light tau sleptons (the staus, labelled as $\tilde{\tau}$ in the following) are consistent with current dark matter searches [35].

This paper presents a search for electroweak production of charginos, next-to-lightest neutralinos and staus in events with at least two hadronically decaying tau leptons, missing transverse momentum and low jet activity, using the 2012 dataset of $\sqrt{s}=8 \mathrm{TeV}$ protonproton collisions collected with the ATLAS detector. Previous searches from the ATLAS collaboration cover electroweak production of supersymmetric particles in final states with electrons and muons [36] using signal models where the neutralinos and charginos decay with equal probability to all lepton flavours, and final states with exactly three leptons of any flavour (electrons, muons, and hadronically decaying taus) [37] using models similar to those described in this paper. A search for associated production of charginos and next-to-lightest neutralinos in stau dominated scenarios has recently been published by the CMS collaboration [38]. The combined LEP limits on the stau and chargino masses are $m_{\tilde{\tau}}>87-93 \mathrm{GeV}$ (depending on the $\tilde{\chi}_{1}^{0}$ mass) and $m_{\tilde{\chi}_{1}^{ \pm}}>103.5 \mathrm{GeV}$ [39-43]. It should be noted that the stau mass limit from LEP assumes gaugino mass unification, which is not assumed in the results presented here.

\section{SUSY scenarios}

SUSY scenarios characterised by the presence of light charginos, next-to-lightest neutralinos and sleptons can be realised in the general framework of the phenomenological Minimal Supersymmetric Standard Model (pMSSM) [44-46]. The dominant processes are the electroweak production of $\tilde{\chi}_{1}^{ \pm} \tilde{\chi}_{2}^{0}$ and $\tilde{\chi}_{1}^{ \pm} \tilde{\chi}_{1}^{\mp}$, such as $q \bar{q} \rightarrow(Z / \gamma)^{*} \rightarrow \tilde{\chi}_{1}^{+} \tilde{\chi}_{1}^{-}$and $q \bar{q}^{\prime} \rightarrow W^{ \pm *} \rightarrow \tilde{\chi}_{1}^{ \pm} \tilde{\chi}_{2}^{0}$. The chargino and neutralino decay properties depend on the Minimal Supersymmetric Standard Model (MSSM) parameters $M_{1}$ and $M_{2}$ (the gaugino masses), $\tan \beta$ (the ratio of the vacuum expectation values of the two Higgs doublets), and $\mu$ (the higgsino-mixing mass term). In this paper we study two pMSSM model implementations

\footnotetext{
${ }^{1}$ The sleptons are referred to as left- or right-handed $\left(\tilde{\ell}_{L}\right.$ or $\left.\tilde{\ell}_{R}\right)$, depending on the helicity of their SM partners. The slepton mass eigenstates are a mixture of $\tilde{\ell}_{L}$ and $\tilde{\ell}_{R}$ and labelled as $\tilde{\ell}_{1}$ and $\tilde{\ell}_{2}$.
} 

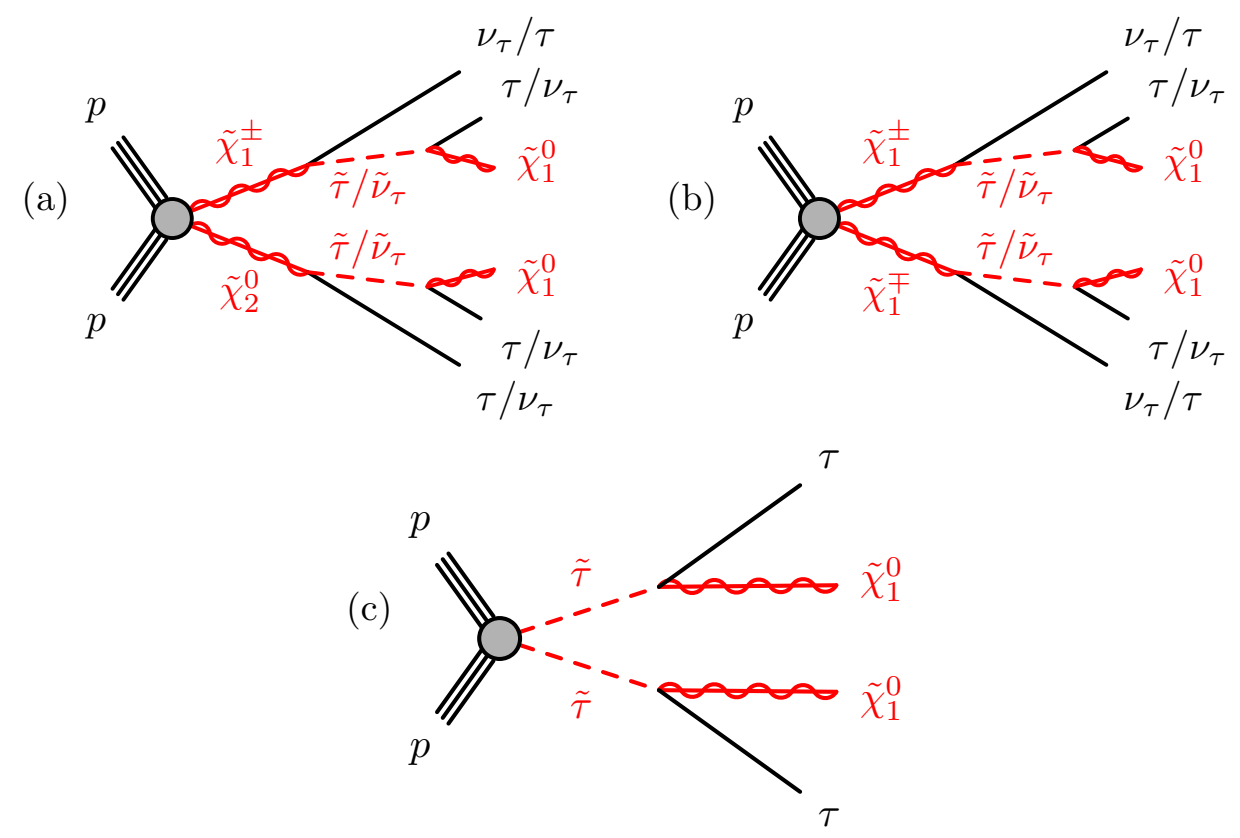

Figure 1. Representative diagrams for the electroweak production processes of supersymmetric particles considered in this work: (a) $\tilde{\chi}_{1}^{ \pm} \tilde{\chi}_{2}^{0}$, (b) $\tilde{\chi}_{1}^{ \pm} \tilde{\chi}_{1}^{\mp}$, and (c) $\tilde{\tau} \tilde{\tau}$ production.

with large $\tan \beta$ and where the only light slepton is the stau partner of the right-handed tau $\left(\tilde{\tau}_{\mathrm{R}}\right)$. More details of the considered models are given in section 5.2.

"Simplified models" $[47,48]$ characterised by $\tilde{\chi}_{1}^{ \pm} \tilde{\chi}_{2}^{0}$ and $\tilde{\chi}_{1}^{ \pm} \tilde{\chi}_{1}^{\mp}$ production, where the charginos and neutralinos decay with $100 \%$ branching fraction to final states with taus, are also considered. In both simplified models the lightest neutralino is the LSP, and the only light slepton is the stau partner of the left-handed tau $\left(\tilde{\tau}_{\mathrm{L}}\right)$. If the stau and the superpartner of the tau neutrino (the tau sneutrino, labelled as $\tilde{\nu}_{\tau}$ ) are lighter than the $\tilde{\chi}_{1}^{ \pm}$and $\tilde{\chi}_{2}^{0}$, the following decay processes can occur: $\tilde{\chi}_{2}^{0} \rightarrow \tilde{\tau}_{\mathrm{L}} \tau \rightarrow \tau \tau \tilde{\chi}_{1}^{0}$, and $\tilde{\chi}_{1}^{ \pm} \rightarrow \tilde{\tau}_{\mathrm{L}} \nu\left(\tilde{\nu}_{\tau} \tau\right) \rightarrow \tau \nu \tilde{\chi}_{1}^{0}$. Figures $1(\mathrm{a})$ and $1(\mathrm{~b})$ show diagrams of associated $\tilde{\chi}_{1}^{ \pm} \tilde{\chi}_{2}^{0}$ and $\tilde{\chi}_{1}^{ \pm} \tilde{\chi}_{1}^{\mp}$ production.

If charginos and next-to-lightest neutralinos are too heavy to be produced at the LHC, direct production of stau pairs [49] might become the dominant electroweak production process in the pMSSM. The production of stau pairs is studied in this paper, and the relevant process is depicted in figure $1(\mathrm{c})$.

The studied final state contains at least two taus with opposite electric charge (opposite-sign, OS), low jet activity and large missing transverse momentum due to the escaping neutrinos and LSPs. Only final states containing hadronically decaying taus are considered in this search.

\section{The ATLAS detector}

The ATLAS detector [50] is a multi-purpose particle physics detector with forwardbackward symmetric cylindrical geometry, and nearly $4 \pi$ coverage in solid angle. ${ }^{2}$ It fea-

\footnotetext{
${ }^{2}$ ATLAS uses a right-handed coordinate system with its origin at the nominal interaction point (IP) in the centre of the detector, and the $z$-axis along the beam line. The $x$-axis points from the IP to the centre of
} 
tures an inner tracking detector (ID) surrounded by a $2 \mathrm{~T}$ superconducting solenoid, electromagnetic and hadronic calorimeters, and a muon spectrometer (MS). The ID covers the pseudorapidity region $|\eta|<2.5$ and consists of a silicon pixel detector, a silicon microstrip detector (SCT), and a transition radiation tracker (TRT). The calorimeters are composed of high-granularity liquid-argon (LAr) electromagnetic calorimeters with lead, copper, or tungsten absorbers (in the pseudorapidity region $|\eta|<3.2$ ) and an iron-scintillator hadronic calorimeter (over $|\eta|<1.7$ ). The end-cap and forward regions, spanning $1.5<|\eta|<4.9$, are instrumented with LAr calorimeters for both the electromagnetic and hadronic measurements. The MS surrounds the calorimeters and consists of three large superconducting air-core toroid magnets, each with eight coils, a system of precision tracking chambers $(|\eta|<2.7)$, and detectors for triggering $(|\eta|<2.4)$. Events are selected by a three-level trigger system.

\section{Data sample}

The analysed dataset, after the application of beam, detector and data quality requirements, corresponds to an integrated luminosity of $20.3 \pm 0.6 \mathrm{fb}^{-1}$. The luminosity is measured using techniques similar to those described in ref. [51] with a preliminary calibration of the luminosity scale derived from beam-overlap scans performed in November 2012. The average number of inelastic interactions per bunch crossing (pile-up) varied from 5.9 to 36.5 .

The events used in this analysis are recorded using a di-tau trigger, which requires identification of two hadronically decaying tau candidates with transverse momenta $\left(p_{\mathrm{T}}\right)$ exceeding a set of thresholds, similar to those described in ref. [52]. Trigger efficiency measurements using a sample of $Z \rightarrow \tau \tau$ events where one tau decays hadronically and the other leptonically into a muon and two neutrinos, show that the di-tau trigger reaches constant efficiency $(\sim 65 \%)$ when the leading tau has $p_{\mathrm{T}}>40 \mathrm{GeV}$ and the next-to-leading tau has $p_{\mathrm{T}}>25 \mathrm{GeV}$.

\section{Monte Carlo simulation}

Monte Carlo (MC) simulated event samples are used to estimate the SUSY signal yields and to aid in evaluating the SM backgrounds. MC samples are processed through a detailed detector simulation [53] based on GEANT4 [54] and reconstructed using the same algorithms as the data. The effect of multiple proton-proton collisions in the same or nearby bunch crossings is also taken into account.

\subsection{Standard Model processes}

The main sources of SM background to final states with at least two hadronically decaying taus are multi-jet, $W+$ jets and diboson events. They are estimated with methods using

the LHC ring, and the $y$-axis points upwards. Cylindrical coordinates $(r, \phi)$ are used in the transverse plane, $\phi$ being the azimuthal angle around the $z$-axis. Observables labelled "transverse" refer to the projection into the $x-y$ plane. The pseudorapidity is defined in terms of the polar angle $\theta$ by $\eta=-\ln \tan (\theta / 2)$. 
simulation samples and data as described in section 8. MC samples are used to estimate the SM background contributions from processes leading to at least one tau from prompt boson decays in the final state, such as diboson production ( $W W, W Z, Z Z)$, processes including a top quark pair or single top quark (in association with jets or $W / Z$ bosons), and $Z$ boson production in association with jets. Production of the SM Higgs boson with a mass of $125 \mathrm{GeV}$ is also considered.

The diboson samples are generated with SHERPA v1.4.1 [55], with additional gluongluon contributions simulated with gg2WW v3.1.2 [56]. The production of top quark pairs is also simulated with the SHERPA v1.4.1 generator. Samples of $t \bar{t}+V(V=W, Z)$ are generated with the leading-order (LO) generator MadGraph 5 v1.3.33 [57] interfaced to PYTHIA v8.165 [58, 59]. Single-top production is simulated with MC@NLO v4.06 (Wt-and $s$-channel) [60-62] and AcerMC v3.8 ( $t$-channel) [63]. In all samples the top quark mass is set to $172.5 \mathrm{GeV}$. Events with $Z / \gamma^{*} \rightarrow \ell \ell$ and $W \rightarrow \ell \nu$ produced with accompanying jets (including light and heavy flavours) are generated with ALPGEN v2.14 [64] interfaced to PYTHIA 6. The gluon fusion and vector-boson fusion production modes of the SM Higgs are simulated with POWHEG-BOX v1.0 [65], and the associated production ( $W H$ and $Z H$ ) with PYTHIA v8.165.

The simulation parameters are tuned to describe the soft component of the hadronic final state [66, 67]. The next-to-leading-order (NLO) CT10 [68] parton distribution function (PDF) set is used for SHERPA and MC@NLO. The CTEQ6L1 [69] set is used for MadGraph, AcerMC, PYTHIA, and ALPGEN.

All SM background production cross sections are normalised to the results of higherorder calculations when available. The inclusive $W$ and $Z$ production cross sections are calculated to next-to-next-to-leading order (NNLO) in the strong coupling constant with DYNNLO [70] using the MSTW2008NNLO PDF set [71]. The $t \bar{t}$ cross section is normalised to a NNLO calculation including resummation of next-to-next-to-leading logarithmic (NNLL) soft gluon terms obtained with Top++ v2.0 [72]. The diboson production cross section is normalised to NLO using MCFM v6.2 [73, 74]. The production of $t \bar{t}$ in association with $W / Z$ is normalised to the NLO cross section $[75,76]$.

\subsection{SUSY processes}

Simulated signal samples are generated with Herwig++ v2.5.2 [77] and the CTEQ6L1 PDF set. Signal production cross sections are calculated to NLO using PROSPINO2 [78]. They are in agreement with the NLO calculations matched to resummation at the next-to-leadinglogarithmic accuracy (NLO+NLL) within $\sim 2 \%$ [79-81].

The results of this search are interpreted in the context of two pMSSM models with the following specifications. The masses of squarks, gluinos and sleptons other than the stau partner of the right-handed taus are set to $3 \mathrm{TeV}$, and $\tan \beta$ is set to 50 . In the first (second) pMSSM model, $M_{1}$ is set to 50 (75) GeV and $M_{2}$ and $\mu$ are varied between 100 and $500(600) \mathrm{GeV}$. The mass of the lighter stau, $\tilde{\tau}_{1}=\tilde{\tau}_{\mathrm{R}}$, is set to $95 \mathrm{GeV}$ in the first pMSSM model, whereas in the second pMSSM model it is set halfway between those of the $\tilde{\chi}_{2}^{0}$ and the $\tilde{\chi}_{1}^{0}$. In the first pMSSM scenario (with fixed stau mass), the dominant processes are the associated production of charginos and neutralinos $\left(\tilde{\chi}_{1}^{ \pm} \tilde{\chi}_{2}^{0}\right)$, or pair production of charginos 
$\left(\tilde{\chi}_{1}^{ \pm} \tilde{\chi}_{1}^{\mp}\right)$ or staus $(\tilde{\tau} \tilde{\tau})$, depending on the values of $M_{2}$ and $\mu$. The cross section of direct stau production is $163 \mathrm{fb}$ over the whole set of models, while the production cross sections of chargino-neutralino and chargino-chargino vary from $5 \cdot 10^{-3}$ to $40 \mathrm{pb}$ and from 0.01 to 16 $\mathrm{pb}$, respectively. In the second pMSSM scenario (with variable stau mass), the dominant processes are $\tilde{\chi}_{1}^{ \pm} \tilde{\chi}_{2}^{0}$ and $\tilde{\chi}_{1}^{ \pm} \tilde{\chi}_{1}^{\mp}$ production. The cross section of direct stau production varies from 0.4 to $42 \mathrm{fb}$, while the production cross sections of chargino-neutralino and chargino-chargino vary from $5 \cdot 10^{-4}$ to $1.2 \mathrm{pb}$ and $8 \cdot 10^{-4}$ to $0.9 \mathrm{pb}$, respectively.

Two simplified models characterised by $\tilde{\chi}_{1}^{ \pm} \tilde{\chi}_{2}^{0}$ and $\tilde{\chi}_{1}^{ \pm} \tilde{\chi}_{1}^{\mp}$ production are also considered. In these models, all sparticles other than $\tilde{\chi}_{1}^{ \pm}, \tilde{\chi}_{2}^{0}, \tilde{\chi}_{1}^{0}, \tilde{\tau}_{\mathrm{L}}$ and $\tilde{\nu}_{\tau}$ are assumed to be heavy (masses of order of $2 \mathrm{TeV}$ ). The neutralinos and charginos decay via intermediate staus and tau sneutrinos. The stau and tau sneutrino are assumed to be mass-degenerate, which happens to be often the case in pMSSM scenarios with large mass splitting between $\tilde{\chi}_{1}^{ \pm}$and $\tilde{\chi}_{1}^{0}$. The mass of the $\tilde{\tau}_{\mathrm{L}}$ state is set to be halfway between those of the $\tilde{\chi}_{1}^{ \pm}$and the $\tilde{\chi}_{1}^{0}$. Furthermore, $\tilde{\chi}_{1}^{ \pm}$and $\tilde{\chi}_{2}^{0}$ are assumed to be pure wino and mass-degenerate, while the $\tilde{\chi}_{1}^{0}$ is purely bino. The $\tilde{\chi}_{1}^{ \pm}\left(\tilde{\chi}_{2}^{0}\right)$ mass is varied between 100 and $500 \mathrm{GeV}$, and the $\tilde{\chi}_{1}^{0}$ mass is varied between zero and $350 \mathrm{GeV}$. The cross section for electroweak production of supersymmetric particles ranges from 0.01 to $2 \mathrm{pb}$ in the considered models.

Direct stau production is studied in the context of the pMSSM model described in ref. [82]. The masses of all charginos and neutralinos apart from the $\tilde{\chi}_{1}^{0}$ are set to $2.5 \mathrm{TeV}$. The model contains $\tilde{\tau}_{\mathrm{R}}$ and $\tilde{\tau}_{\mathrm{L}}$, but no tau sneutrinos. The stau mixing is set such that $\tilde{\tau}_{1}=\tilde{\tau}_{\mathrm{R}}$ and $\tilde{\tau}_{2}=\tilde{\tau}_{\mathrm{L}}$. The stau masses are generated in the range from 90 to $300 \mathrm{GeV}$ and the mass of the bino-like $\tilde{\chi}_{1}^{0}$ is varied by scanning the gaugino mass parameter $M_{1}$ in the range from zero to $200 \mathrm{GeV}$. The cross section for direct stau pair production in this scenario decreases from 176 to $1.4 \mathrm{fb}$ for $\tilde{\tau}_{\mathrm{L}}$, and from 70 to $0.6 \mathrm{fb}$ for $\tilde{\tau}_{\mathrm{R}}$ as the stau mass increases from 90 to $300 \mathrm{GeV}$.

Three reference points are used throughout this paper to illustrate the typical features of the SUSY models to which this analysis is sensitive:

- Ref. point 1: simplified model for chargino-neutralino production with mass of $\tilde{\chi}_{1}^{ \pm}$ $\left(\tilde{\chi}_{2}^{0}\right)$ equal to $250 \mathrm{GeV}$, and mass of $\tilde{\chi}_{1}^{0}$ equal to $100 \mathrm{GeV}$;

- Ref. point 2: simplified model for chargino-chargino production with mass of $\tilde{\chi}_{1}^{ \pm}$ equal to $250 \mathrm{GeV}$, and mass of $\tilde{\chi}_{1}^{0}$ equal to $50 \mathrm{GeV}$;

- Ref. point 3: direct stau production with mass of the $\tilde{\tau}_{\mathrm{R}}\left(\tilde{\tau}_{\mathrm{L}}\right)$ equal to 127 (129) GeV, and massless $\tilde{\chi}_{1}^{0}$.

\section{Event reconstruction}

Events with at least one reconstructed primary vertex are selected. A primary vertex must have at least five associated charged-particle tracks with $p_{\mathrm{T}}>400 \mathrm{MeV}$ and be consistent with the beam spot envelope. If there are multiple primary vertices in an event, the one with the largest $\sum p_{\mathrm{T}}^{2}$ of the associated tracks is chosen. 
Jets are reconstructed from three-dimensional calorimeter energy clusters using the anti- $k_{t}$ algorithm $[83,84]$ with a radius parameter of 0.4 . Jet energies are corrected for detector inhomogeneities, the non-compensating nature of the calorimeter, and the impact of pile-up, using factors derived from test beam, cosmic ray, and $p p$ collision data, and from a detailed GEANT4 detector simulation [85]. The impact of pile-up is accounted for by using a technique, based on jet areas, that provides an event-by-event and jet-by-jet correction [86]. Events containing jets that are likely to have arisen from detector noise or cosmic rays are removed. For this analysis jets are required to have $p_{\mathrm{T}}>20 \mathrm{GeV}$ and $|\eta|<4.5$.

Electron candidates are reconstructed by matching clusters in the electromagnetic calorimeter with charged particle tracks in the inner detector. Electrons are required to have $p_{\mathrm{T}}>10 \mathrm{GeV},|\eta|<2.47$, and to satisfy the "medium" shower-shape and trackselection criteria defined in ref. [87], updated for the 2012 operating conditions. Muon candidates are identified by matching an extrapolated inner detector track and one or more track segments in the muon spectrometer [88]. Muons are required to have $p_{\mathrm{T}}>10 \mathrm{GeV}$ and $|\eta|<2.4$. Events with muons compatible with cosmic rays are rejected.

The reconstruction of hadronically decaying taus is based on the information from tracking in the ID and three-dimensional clusters in the electromagnetic and hadronic calorimeters. The tau reconstruction algorithm is seeded by jets reconstructed as described above but with $p_{\mathrm{T}}>10 \mathrm{GeV}$ and $|\eta|<2.47$. Tracks are subsequently associated with the tau jet within a cone of size $\Delta R=\sqrt{(\Delta \eta)^{2}+(\Delta \phi)^{2}}=0.2$ around the axis of the tau cluster. The reconstructed energies of the hadronically decaying tau candidates are corrected to the tau energy scale, which is calibrated independently of the jet energy scale, by a MC-based procedure [89]. Tau neutrinos from the tau lepton decay are not taken into account in the reconstruction and calibration of the tau energy and momentum. Since taus decay mostly to either one or three charged pions, together with a neutrino and often additional neutral pions, tau candidates are required to have one or three associated charged particle tracks (prongs) and the total electric charge of those tracks must be \pm 1 times the electron charge. To improve the discrimination between hadronically decaying taus and jets, electrons, or muons, multivariate algorithms are used [90]. The tau identification algorithm used in this analysis is based on the Boosted Decision Tree (BDT) method. The BDT algorithms use as input various track and cluster variables for particle discrimination. A "jet BDT" is used to discriminate taus from jets, and an "electron BDT" to discriminate between electrons and taus. Based on the jet BDT result, three tau identification criteria corresponding to "loose", "medium", and "tight" quality can be defined. For 1-prong (3-prong) taus the signal efficiencies are $70 \%, 60 \%$ and $40 \%(65 \%, 55 \%$ and $35 \%)$ for the loose, medium and tight working points, respectively. Background rejection factors ranging from 10 to 40 for signal efficiencies of $70 \%$ are achieved, increasing to 500 for $35 \%$ signal efficiency. In the following, tau candidates are required to pass the "medium" identification criteria for jet discrimination, while for the final signal region selections both the "tight" and "medium" criteria are used. For electron discrimination, the "loose" quality selection is applied to 1-prong taus only. This requirement has about $95 \%$ efficiency, and a rejection factor from 10 to 50 depending on the $\eta$ range. In addition, a dedicated muon veto is 
applied to remove tau candidates generated by muons associated with anomalous energy deposits in the calorimeter. The resulting signal efficiency is better than $96 \%$, with a reduction of muons misidentified as taus of around $40 \%$. Tau candidates are required to have $p_{\mathrm{T}}>20 \mathrm{GeV}$ and $|\eta|<2.47$.

The measurement of the missing transverse momentum two-vector, $\mathbf{p}_{\mathrm{T}}^{\text {miss }}$, and its magnitude, $E_{\mathrm{T}}^{\text {miss }}$, is based on the vectorial sum of the $\mathbf{p}_{\mathrm{T}}$ of reconstructed objects (jets, taus, electrons, photons, muons) as well as calorimeter energy clusters (with $|\eta|<4.9$ ) not associated with reconstructed objects [91]. Since different requirements on the tau identification (loose, medium, and tight jet BDT quality requirements) are used throughout this analysis, taus are calibrated at the jet energy scale for the calculation of $\mathbf{p}_{\mathrm{T}}^{\text {miss }}$.

The possible double counting of reconstructed objects is resolved in the following order. If two electron candidates are found within a distance $\Delta R=0.05$, the electron candidate with lower momentum is discarded. Jet candidates are removed if they lie within a distance $\Delta R=0.2$ of a tau or an electron. Any tau candidate lying within a distance $\Delta R=0.2$ of any remaining electron or muon is discarded. Any muon candidate within $\Delta R=0.4$ of a jet is removed. The remaining electrons are rejected if they lie within $0.2<\Delta R<0.4$ of a jet. Electron and muon candidates within a distance $\Delta R=0.1$, or muon candidates within a distance $\Delta R=0.05$, are rejected. To remove low-mass resonances, electron or muon pairs with opposite electrical charge are rejected if their invariant mass is less than $12 \mathrm{GeV}$.

Jets are further classified as jets containing a $b$-quark ( $b$-jets), light-parton jets, and forward jets. A $b$-tagging algorithm [92], which exploits the long lifetime of $b$-hadrons, is used to identify jets containing a $b$-quark. The mean nominal $b$-tagging efficiency, determined from $t \bar{t} \mathrm{MC}$ events, is $80 \%$, with a misidentification (mis-tag) rate for light-quark/gluon jets of less than $1 \%$. Correction factors are applied as functions of the $p_{\mathrm{T}}$ and $\eta$ of the jets to all MC samples to correct for small differences in the $b$-tagging performance observed between data and simulation. Jets in the central region, satisfying $p_{\mathrm{T}}>20 \mathrm{GeV},|\eta|<2.4$, and the $b$-tagging algorithm, are defined as $b$-jets, or B20. Central light jets are required to have $|\eta|<2.4$, and not be identified as $b$-jets. To remove jets that originated from pile-up collisions, a central light jet with $p_{\mathrm{T}}<50 \mathrm{GeV}$ must have at least one track with $p_{\mathrm{T}}>400 \mathrm{MeV}$ associated with it and with the primary vertex of the event. Depending on their transverse momentum, central light jets are referred to as L30 (L50) if they have $p_{\mathrm{T}}>30(50) \mathrm{GeV}$. Forward jets, or F30, must satisfy $p_{\mathrm{T}}>30 \mathrm{GeV}$ and $2.4<|\eta|<4.5$.

For the background estimation and validation described in section 8 , events containing isolated electrons or muons are selected. Isolated electrons and muons are defined as follows. The summed scalar $p_{\mathrm{T}}$ of tracks above $400 \mathrm{MeV}$ within a cone of size $\Delta R=0.3$ around each electron or muon candidate (excluding the candidate itself) and associated with the primary vertex is required to be less than $16 \%$ of the electron or muon $p_{\mathrm{T}}$. The distance of closest approach in the transverse plane of an electron or muon candidate to the event primary vertex must be within five (for electron candidates) or three (for muon candidates) standard deviations from its measurement in the transverse plane. For isolated electrons, the sum of transverse energies of the surrounding calorimeter energy clusters within $\Delta R=0.3$ of each electron candidate, corrected for the deposition of energy from pile-up interactions, is required to be less than $18 \%$ of the electron $p_{\mathrm{T}}$. The longitudinal 
impact parameter of an electron, $z_{0}$, must satisfy $\left|z_{0} \sin \theta\right|<0.4 \mathrm{~mm}$. Muon candidates are required to satisfy $\left|z_{0} \sin \theta\right|<0.1 \mathrm{~mm}$. Furthermore, isolated electrons must satisfy the "tight" criteria $[87,93]$ placed on the ratio of calorimetric energy to track momentum, and the number of high-threshold hits in the TRT.

The simulation is corrected for differences in the efficiency of the tau identification and trigger algorithms between data and MC simulation. For hadronically decaying taus coming from prompt boson decays, the corrections are calculated with a "tag-and-probe" method in a sample of $Z \rightarrow \tau \tau$ events where one tau decays hadronically and the other leptonically into a muon and two neutrinos [90]. For misidentified taus from electrons a sample of $Z \rightarrow e e$ events is used, while the mis-identification rate of taus from light jets is measured in a sample of $Z \rightarrow \mu \mu$ events with associated jets. The efficiencies for electrons and muons to satisfy the reconstruction, identification and isolation criteria are measured in samples of $Z$ and $J / \psi$ leptonic decays, and corrections are applied to the simulated samples to reproduce the efficiencies in data.

\section{Event selection}

Events are required to have at least two candidate taus, and at least one must satisfy the tight jet BDT quality requirement. At least one of the selected tau pairs must contain taus with opposite electrical charge. Two of the reconstructed taus must have fired the di-tau trigger, and satisfy the $p_{\mathrm{T}}$ requirements to be in the region where the trigger efficiency is constant, i.e. the more (less) energetic tau must have $p_{\mathrm{T}}>40$ (25) $\mathrm{GeV}$. The di-tau invariant mass of any opposite-sign (OS) pair must be larger than $12 \mathrm{GeV}$ to remove taus from low-mass resonances. This requirement has negligible effect on the signal efficiency. Events with additional light leptons (defined as candidate electrons or muons after resolving the overlap between the various reconstructed objects as described in section 6) are vetoed to allow for a statistical combination with other ATLAS analyses [36, 37].

To enhance the sensitivity to the SUSY signal and suppress SM backgrounds, additional requirements are applied that define the so-called signal regions (SR). At tree level, no jet is present in the SUSY processes of interest; however, jets can be generated from initial-state radiation (ISR). Vetoing events containing jets suppresses background contributions involving top quarks but may also reduce the signal efficiency due to ISR. To maximise the signal-to-background ratio, three event-based jet vetoes are used depending on the SR: a " $b$-jet veto", where only events with no $b$-jets, i.e. $N($ B20 $)=0$, are accepted; a "jet veto", where only events with $N($ B20 $)+N($ L30 $)+N($ F30 $)=0$ are selected; and a "looser jet-veto", where $N(\mathrm{~B} 20)+N(\mathrm{~L} 50)+N($ F30 $)=0$ is required. The rejection factors of the $b$-jet veto, jet veto, and looser jet-veto measured on MC samples of top quark backgrounds $(t \bar{t}$, single top and $t \bar{t}+V)$ are about 6,50 , and 20 , respectively.

To reject backgrounds with a $Z$ boson, events where at least one of the oppositely charged tau pairs has a reconstructed invariant mass within $10 \mathrm{GeV}$ of the visible $Z$ boson mass $(81 \mathrm{GeV})$ are vetoed. The visible $Z$ boson mass is obtained from the mean value of a gaussian fit of the reconstructed invariant mass distribution of OS tau pairs in a MC sample of $Z(\rightarrow \tau \tau)+$ jets events. This requirement is referred to as the " $Z$-veto". The $Z$ - 
veto reduces the contribution from background processes containing $Z$ decays by a factor of three. An additional requirement on the angular separation between the leading and next-to-leading tau, $\Delta R(\tau, \tau)<3$, is effective in discriminating against back-to-back events such as multi-jet production or $Z$ decays.

To further improve the signal to background ratio, additional requirements on a selection of sensitive kinematic variables are applied depending on the SR. The "stransverse" mass $m_{\mathrm{T} 2}[94,95]$ is defined as:

$$
m_{\mathrm{T} 2}=\min _{\mathbf{q}_{\mathrm{T}}}\left[\max \left(m_{\mathrm{T}}\left(\mathbf{p}_{\mathrm{T}}^{\tau 1}, \mathbf{q}_{\mathrm{T}}\right), m_{\mathrm{T}}\left(\mathbf{p}_{\mathrm{T}}^{\tau 2}, \mathbf{p}_{\mathrm{T}}^{\mathrm{miss}}-\mathbf{q}_{\mathrm{T}}\right)\right)\right],
$$

where $\mathbf{p}_{\mathrm{T}}^{\tau 1}$ and $\mathbf{p}_{\mathrm{T}}^{\tau 2}$ are the transverse momenta of the two taus, and $\mathbf{q}_{\mathrm{T}}$ is a transverse vector that minimises the larger of the two transverse masses $m_{\mathrm{T}}$. The latter is defined by

$$
m_{\mathrm{T}}\left(\mathbf{p}_{\mathrm{T}}, \mathbf{q}_{\mathrm{T}}\right)=\sqrt{2\left(p_{\mathrm{T}} q_{\mathrm{T}}-\mathbf{p}_{\mathrm{T}} \cdot \mathbf{q}_{\mathrm{T}}\right)} .
$$

In events where more than two taus are selected, $m_{\mathrm{T} 2}$ is computed among all possible tau pairs and the combination leading to the largest value is chosen. For $t \bar{t}$ and $W W$ events, in which two on-shell $W$ bosons decay leptonically and $\mathbf{p}_{\mathrm{T}}^{\text {miss }}$ is the sum of the transverse momenta of the two neutrinos, the $m_{\mathrm{T} 2}$ distribution has an upper end-point at the $W$ mass. For large mass differences between the next-to-lightest neutralinos, the charginos, or the staus and the lightest neutralino, the $m_{\mathrm{T} 2}$ distribution for signal events extends significantly beyond the distributions of the $t \bar{t}$ and $W W$ events.

Two additional kinematic variables, sensitive to the additional missing transverse momentum due to the LSPs, are used to discriminate SUSY from SM events. These are the effective mass $m_{\mathrm{eff}}=E_{\mathrm{T}}^{\text {miss }}+p_{\mathrm{T}}^{\tau 1}+p_{\mathrm{T}}^{\tau 2}$, defined as the scalar sum of the missing transverse energy and the transverse momenta of the two leading taus, and $m_{\mathrm{T} \tau 1}+m_{\mathrm{T} \tau 2}$, where $m_{\mathrm{T} \tau 1}\left(m_{\mathrm{T} \tau 2}\right)$ is the transverse mass computed from the transverse momentum of the leading (next-to-leading) tau and $\mathbf{p}_{\mathrm{T}}^{\mathrm{miss}}$. The correlation among the above defined kinematical variables varies between $20 \%$ and $90 \%$ according to the signal model.

Four SRs are defined in this analysis, targeting various SUSY production processes. ${ }^{3}$ The requirements for each SR are summarised in table 1. At least two OS taus are required in all SRs except for SR-C1C1, where exactly two OS taus are required. The first two SRs, SR-C1N2 and SR-C1C1, target $\tilde{\chi}_{1}^{ \pm} \tilde{\chi}_{2}^{0}$ and $\tilde{\chi}_{1}^{ \pm} \tilde{\chi}_{1}^{\mp}$ production, respectively. SR-C1N2 is based on moderate $E_{\mathrm{T}}^{\text {miss }}$ and large $m_{\mathrm{T} 2}$ requirements. In SR-C1C1, events with moderate $m_{\mathrm{T} 2}$ and large $m_{\mathrm{T} \tau 1}+m_{\mathrm{T} \tau 2}$ are selected. The last two SRs, SR-DS-highMass and SRDS-lowMass, are designed to cover direct stau production and are optimised for different ranges of the stau mass. The four SRs are not mutually exclusive.

The signal acceptance for events passing all analysis requirements is of order a few percent for SUSY models to which this analysis is sensitive in all SRs. The trigger efficiency varies between $55 \%$ and $70 \%$ according to the signal region and the SUSY model considered, while the total reconstruction efficiency is about $15 \%$.

\footnotetext{
${ }^{3}$ In the SR definitions, the following mnemonic naming conventions are used: "C1" stands for $\tilde{\chi}_{1}^{ \pm}$, "N2" for $\tilde{\chi}_{2}^{0}$, and "DS" for direct stau production.
} 


\begin{tabular}{|c|c|c|c|}
\hline SR-C1N2 & SR-C1C1 & SR-DS-highMass & SR-DS-lowMass \\
\hline$\geq 2$ OS taus & 2 OS taus & $\geq 2$ OS taus & $\geq 2$ OS taus \\
$b$-jet veto & jet veto & looser jet-veto & looser jet-veto \\
$Z$-veto & $Z$-veto & $Z$-veto & $Z$-veto \\
& & $\Delta R(\tau, \tau)<3$ & $\Delta R(\tau, \tau)<3$ \\
$E_{\mathrm{T}}^{\text {miss }}>40 \mathrm{GeV}$ & $m_{\mathrm{T} 2}>30 \mathrm{GeV}$ & $m_{\mathrm{T} 2}>60 \mathrm{GeV}$ & $m_{\mathrm{T} 2}>30 \mathrm{GeV}$ \\
$m_{\mathrm{T} 2}>100 \mathrm{GeV}$ & $m_{\mathrm{T} \tau 1}+m_{\mathrm{T} \tau 2}>250 \mathrm{GeV}$ & $m_{\mathrm{eff}}>230 \mathrm{GeV}$ & $m_{\mathrm{eff}}>260 \mathrm{GeV}$ \\
\hline
\end{tabular}

Table 1. Signal region definitions.

\section{Standard Model background estimation}

The main SM processes contributing to the selected final states are multi-jet, $W+$ jets and diboson production. Decays of the SM Higgs boson, assuming a mass of $125 \mathrm{GeV}$, into a $\tau \tau$ final state have negligible contribution in all SRs (less than 0.1\%). Background events may contain a combination of 'real' taus, defined as correctly identified tau leptons, or 'fake' taus, which can originate from a misidentified light-flavour quark or gluon jet, an electron or a muon.

In multi-jet events all tau candidates are misidentified jets. Due to the large cross section and the poor MC modelling of the tau mis-identification rate from jets, the multi-jet contribution in the SRs is estimated from data, as described in section 8.1. The contribution arising from heavy-flavour multi-jet events containing a real tau lepton from the heavyflavour quark decay is included in the multi-jet estimate. The contribution of $W+$ jets events, which contain one real tau from the $W$ decay and one or more misidentified jets, is estimated from MC simulation, and normalised to data in a dedicated control region, as described in section 8.2 .

Diboson production contributes mainly with events containing real tau leptons coming from $W W$ and $Z Z$ decaying into a $\tau \tau \nu \nu$ final state. Additional SM backgrounds arise from $Z+$ jets production, or events which contain a top quark or top quark pair in association with jets or additional $W$ or $Z$ bosons (collectively referred to as 'top' background in the following). The contribution from real taus exceeds $90 \%$ in $Z+$ jets and diboson production, and ranges from $45 \%$ to $75 \%$ in backgrounds containing top quarks. The contribution of fake taus from heavy-flavour decays in jets is negligible. To estimate the irreducible background, which includes diboson, $Z+$ jets and top quark events, only MC simulated samples are used, as described in section 8.3. The available MC sample sizes in the SRs are limited for $Z+$ jets and top backgrounds. To improve the statistical precision, the prediction in the SRs for these processes is extrapolated from regions with large MC statistics.

Finally, for each signal region, a simultaneous fit based on the profile likelihood method [96] is performed to normalise the multi-jet and $W+$ jets background estimates, as described in section 8.4. Details of the sources of systematic uncertainty on the background estimates described in this section are given in section 9 . 


\begin{tabular}{|c|c|c|c|}
\hline Regions & $\mathrm{A}$ & B & $\mathrm{C}$ \\
\hline $\begin{array}{c}\text { CR- } \\
\text { C1N2 }\end{array}$ & $\begin{array}{c}m_{\mathrm{T} 2}>100 \mathrm{GeV} \\
\text { at least } 2 \text { loose taus } \\
\text { tight tau veto }\end{array}$ & $\begin{array}{c}m_{\mathrm{T} 2}<40 \mathrm{GeV} \\
\text { at least } 2 \text { loose taus } \\
\text { tight tau veto }\end{array}$ & $\begin{array}{l}m_{\mathrm{T} 2}<40 \mathrm{GeV} \\
\text { at least } 1 \text { medium tau } \\
\text { at least } 1 \text { tight tau }\end{array}$ \\
\hline $\begin{array}{l}\text { CR- } \\
\mathrm{C} 1 \mathrm{C} 1\end{array}$ & $\begin{array}{c}m_{\mathrm{T} \tau 1}+m_{\mathrm{T} \tau 2}>250 \mathrm{GeV} \\
\text { at least } 2 \text { loose taus } \\
\text { tight tau veto }\end{array}$ & $\begin{array}{c}80<m_{\mathrm{T} \tau 1}+m_{\mathrm{T} \tau 2}<150 \mathrm{GeV} \\
\text { at least } 2 \text { loose taus } \\
\text { tight tau veto }\end{array}$ & $\begin{array}{c}80<m_{\mathrm{T} \tau 1}+m_{\mathrm{T} \tau 2}<150 \mathrm{GeV} \\
\text { at least } 1 \text { medium tau } \\
\text { at least } 1 \text { tight tau }\end{array}$ \\
\hline $\begin{array}{l}\text { CR-DS- } \\
\text { highMass }\end{array}$ & $\begin{array}{c}m_{\mathrm{eff}}>230 \mathrm{GeV} \\
\text { at least } 2 \text { loose taus } \\
\text { tight tau veto }\end{array}$ & $\begin{array}{c}130 \mathrm{GeV}<m_{\mathrm{eff}}<150 \mathrm{GeV} \\
\text { at least } 2 \text { loose taus } \\
\text { tight tau veto }\end{array}$ & $\begin{array}{c}130 \mathrm{GeV}<m_{\mathrm{eff}}<150 \mathrm{GeV} \\
\text { at least } 1 \text { medium tau } \\
\text { at least } 1 \text { tight tau }\end{array}$ \\
\hline $\begin{array}{l}\text { CR-DS- } \\
\text { lowMass }\end{array}$ & $\begin{array}{c}m_{\mathrm{eff}}>260 \mathrm{GeV} \\
\text { at least } 2 \text { loose taus } \\
\text { tight tau veto }\end{array}$ & $\begin{array}{c}100 \mathrm{GeV}<m_{\mathrm{eff}}<150 \mathrm{GeV} \\
\text { at least } 2 \text { loose taus } \\
\text { tight tau veto }\end{array}$ & $\begin{array}{c}100 \mathrm{GeV}<m_{\mathrm{eff}}<150 \mathrm{GeV} \\
\text { at least } 1 \text { medium tau } \\
\text { at least } 1 \text { tight tau }\end{array}$ \\
\hline
\end{tabular}

Table 2. The multi-jet control region definitions. Only those requirements that are different in the CRs with respect to the SRs are listed.

\subsection{Multi-jet background estimation}

One of the dominant backgrounds in the SRs originates from jets misidentified as taus in multi-jet production (from $13 \%$ to $30 \%$ of the total background, depending on the SR). This contribution is estimated from data using the "ABCD" method. Four exclusive regions, labelled as A, B, C (the "control regions") and D (the SR), are defined in a two-dimensional plane as a function of two uncorrelated discriminating variables. In this case, the ratio of the numbers of events in the control regions $(\mathrm{CR}) \mathrm{A}$ and $\mathrm{B}$ equals that of SR D to control region $\mathrm{C}$ : the number of events in the $\mathrm{SR} \mathrm{D}, N_{\mathrm{D}}$, can therefore be calculated from that in control region $\mathrm{A}, N_{\mathrm{A}}$, multiplied by the transfer factor $\mathrm{T}=\mathrm{N}_{\mathrm{C}} / \mathrm{N}_{\mathrm{B}}$.

The tau identification criterion (tau-id) based on the jet BDT quality requirement and a kinematic variable chosen depending on the SR are used as the two discriminating variables to define the regions $\mathrm{A}, \mathrm{B}, \mathrm{C}$ and $\mathrm{D}$. The following kinematic variables are used: $m_{\mathrm{T} 2}$ for SR-C1N2, $m_{\mathrm{T} \tau 1}+m_{\mathrm{T} \tau 2}$ for SR-C1C1, and $m_{\text {eff }}$ for SR-DS-highMass and SR-DS-lowMass.

The control region $\mathrm{A}$ and the signal region $\mathrm{D}$ are defined in the same way except that in the control region A all candidate taus must satisfy the "loose" but fail the "tight" jet BDT requirement (tight tau event veto). The same requirement on the tau-id as in control region A (signal region $\mathrm{D}$ ) is applied in control region $\mathrm{B}$ (control region $\mathrm{C}$ ). In control regions $\mathrm{B}$ and $\mathrm{C}$, less stringent requirements on the kinematic variables defined above are applied. The definitions of the control regions are summarised in table 2.

Furthermore, two validation regions $\mathrm{E}$ and $\mathrm{F}$ are defined. The validation region $\mathrm{E}(\mathrm{F})$ has the same definition as the control region A (signal region D) except for intermediate requirements on the kinematic variable, as listed in table 3 . The validation regions are used to verify the extrapolation of the $\mathrm{ABCD}$ estimation to the SR, and to estimate the systematic uncertainty from the residual correlation between the tau-id and the kinematic variable. The regions A-F are drawn schematically in figure 2.

The number of multi-jet events in the control and validation regions is estimated from data after subtraction of other SM contributions estimated from MC simulation. 


\begin{tabular}{|c|c|}
\hline Regions & E/F \\
\hline VR-C1N2 & $40 \mathrm{GeV}<m_{\mathrm{T} 2}<100 \mathrm{GeV}$ \\
VR-C1C1 & $150 \mathrm{GeV}<m_{\mathrm{T} \tau 1}+m_{\mathrm{T} \tau 2}<250 \mathrm{GeV}$ \\
VR-DS-highMass & $150 \mathrm{GeV}<m_{\mathrm{eff}}<230 \mathrm{GeV}$ \\
VR-DS-lowMass & $150 \mathrm{GeV}<m_{\text {eff }}<260 \mathrm{GeV}$ \\
\hline
\end{tabular}

Table 3. The requirement on the kinematic variables used to define the validation regions $\mathrm{E}$ and F. Only those requirements that are different with respect to the A, B, and C CRs and the SRs are listed.

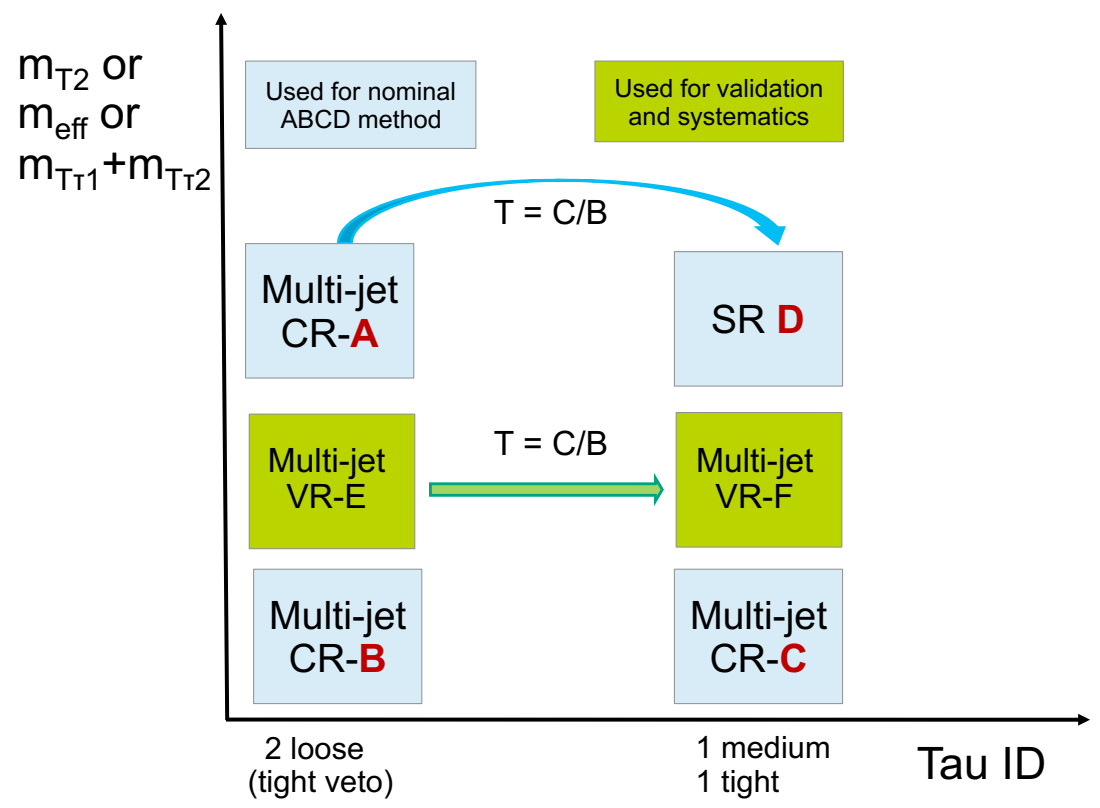

Figure 2. Illustration of the ABCD method for the multi-jet background determination. The control regions A, B, C, and signal region D for the ABCD method described in the text (labelled as Multi-jet CR-A/B/C and SR D) are drawn as light blue boxes. Shown in green and labelled as Multi-jet-VR are the regions $\mathrm{E}$ and $\mathrm{F}$, which are used to validate the ABCD method and to estimate the systematic uncertainties.

Over $80 \%$ of the events contributing to the control regions $\mathrm{B}$ and $\mathrm{C}$ come from multi-jet production. In control region A the multi-jet purity ranges from $60 \%$ to $70 \%$, except for SR-DS-highMass for which the purity is $35 \%$. The prediction in this region is affected by a large statistical uncertainty.

The distributions of the kinematic variables in the control regions $\mathrm{A}, \mathrm{B}$ and in validation region $\mathrm{E}$ are shown in figure 3. The results of the ABCD method are summarised in table 4 . The SM predictions are in agreement with the observed data counts in the multijet validation regions, as shown in table 5 . 


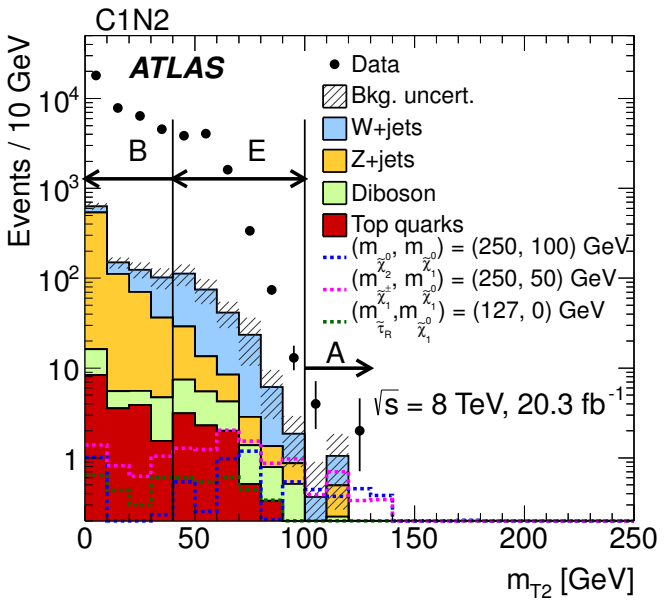

(a)

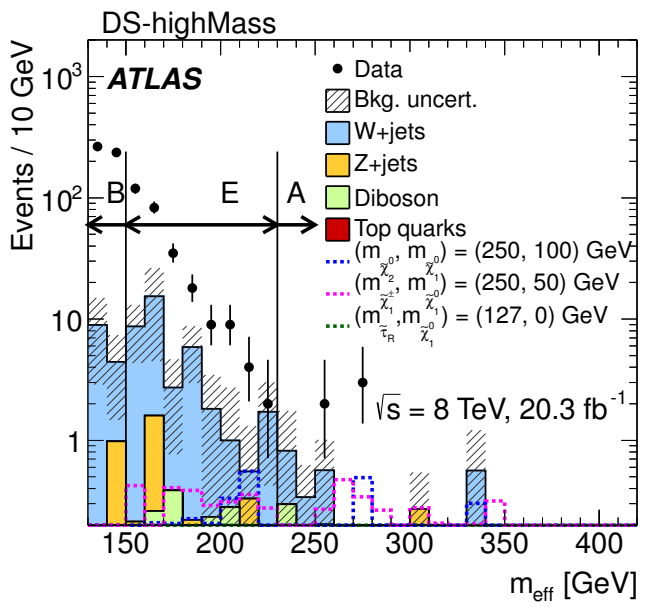

(c)

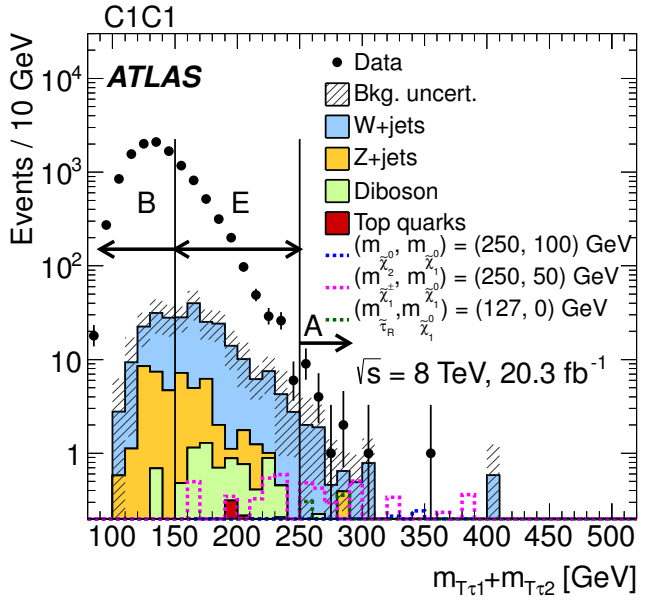

(b)

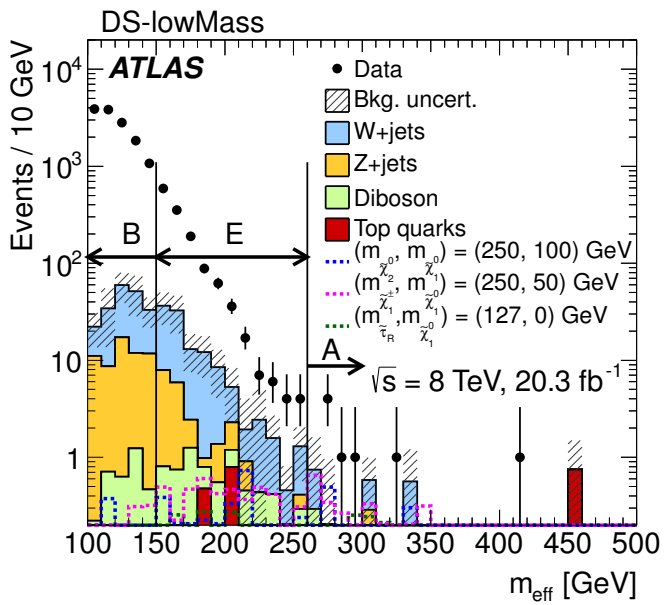

(d)

Figure 3. (a) $m_{\mathrm{T} 2}$, (b) $m_{\mathrm{T} \tau 1}+m_{\mathrm{T} \tau 2}$ and (c,d) $m_{\text {eff }}$ distributions in the multi-jet background CRs $\mathrm{A}$ and $\mathrm{B}$ and in the validation region $\mathrm{E}$ defined in tables 2 and 3 . The stacked histograms show the contribution of the non-multi-jet SM backgrounds from MC simulation, normalised to $20.3 \mathrm{fb}^{-1}$. The hatched bands represent the combined statistical and systematic uncertainties on the sum of the SM backgrounds shown. For illustration, the distributions of the SUSY reference points (see section 5.2) are also shown as dashed lines.

\subsection{W+jets background estimation}

The production of $W+$ jets events with at least one misidentified tau is an important background in the SRs, ranging from $25 \%$ to $50 \%$. A dedicated control region $(W \mathrm{CR}$ ) is used to normalise the $W+$ jets $\mathrm{MC}$ estimation to data. The $W$ CR is designed to be kinematically as close as possible to the signal regions, and is enriched in events where the $W$ decays leptonically into a muon and a neutrino to suppress multi-jet contamination. Events containing exactly one isolated muon and one tau passing the tight jet BDT requirement are selected. The selected leptons must have opposite electrical charge. To reduce the contribution from $Z+$ jets production, $m_{\mathrm{T}, \tau}+m_{\mathrm{T}, \mu}>80 \mathrm{GeV}$ is required, and 


\begin{tabular}{|c|c|c|c|c|c|c|}
\hline & Sample & Region A & Region B & Region $\mathrm{C}$ & $\mathrm{T}=\mathrm{C} / \mathrm{B}$ & Multi-jet in SR (D) \\
\hline \multirow{7}{*}{$\mathrm{C} 1 \mathrm{~N} 2$} & Data & 6 & 36907 & 24601 & \multirow{7}{*}{$\begin{array}{c}0.55 \\
\pm 0.03\end{array}$} & \multirow{7}{*}{$\begin{array}{c}2.3 \\
\pm 1.4\end{array}$} \\
\hline & $Z+$ jets & $0.28 \pm 0.16$ & $730 \pm 260$ & $3980 \pm 1060$ & & \\
\hline & $W+$ jets & $1.0 \pm 0.4$ & $250 \pm 82$ & $590 \pm 180$ & & \\
\hline & diboson & $0.51 \pm 0.26$ & $14.6 \pm 4.8$ & $72 \pm 20$ & & \\
\hline & top & $0.10 \pm 0.06$ & $17.3 \pm 6.1$ & $68 \pm 22$ & & \\
\hline & multi-jet & $4.1 \pm 2.5$ & $35900 \pm 330$ & $19890 \pm 1090$ & & \\
\hline & Ref. point 1 & $1.9 \pm 0.9$ & $1.4 \pm 0.7$ & $17.8 \pm 6.2$ & & \\
\hline \multirow{7}{*}{$\mathrm{C} 1 \mathrm{C} 1$} & Data & 18 & 8479 & 4551 & \multirow{7}{*}{$\begin{array}{c}0.51 \\
\pm 0.01\end{array}$} & \multirow{7}{*}{$\begin{array}{c}5.8 \\
\pm 2.3\end{array}$} \\
\hline & $Z+$ jets & $0.06 \pm 0.06$ & $21 \pm 10$ & $80 \pm 25$ & & \\
\hline & $W+$ jets & $5.6 \pm 1.2$ & $71 \pm 32$ & $160 \pm 46$ & & \\
\hline & diboson & $0.9 \pm 0.4$ & $1.2 \pm 0.6$ & $5.4 \pm 2.2$ & & \\
\hline & top & $0.11 \pm 0.06$ & $0.0 \pm 1.0$ & $0.6 \pm 0.5$ & & \\
\hline & multi-jet & $11.3 \pm 4.4$ & $8390 \pm 98$ & $4300 \pm 85$ & & \\
\hline & Ref. point 2 & $3.9 \pm 1.0$ & $0.13 \pm 0.09$ & $1.4 \pm 0.4$ & & \\
\hline \multirow{7}{*}{$\begin{array}{c}\text { DS- } \\
\text { highMass }\end{array}$} & Data & 5 & 500 & 268 & \multirow{7}{*}{$\begin{array}{c}0.50 \\
\pm 0.05\end{array}$} & \multirow{7}{*}{$\begin{array}{c}0.9 \\
\pm 1.2\end{array}$} \\
\hline & $Z+$ jets & $0.24 \pm 0.18$ & $1.0 \pm 0.7$ & $1.7 \pm 1.0$ & & \\
\hline & $W+$ jets & $2.2 \pm 0.7$ & $12.3 \pm 6.6$ & $20.6 \pm 9.6$ & & \\
\hline & diboson & $0.7 \pm 0.4$ & $0.2 \pm 0.1$ & $0.6 \pm 0.3$ & & \\
\hline & top & $0.06 \pm 0.03$ & $0.0 \pm 1.0$ & $0.8 \pm 0.6$ & & \\
\hline & multi-jet & $1.8 \pm 2.4$ & $487 \pm 23$ & $244 \pm 19$ & & \\
\hline & Ref. point 3 & $0.9 \pm 0.4$ & $0.0 \pm 1.0$ & $0.20 \pm 0.13$ & & \\
\hline \multirow{7}{*}{$\begin{array}{c}\text { DS- } \\
\text { lowMass }\end{array}$} & Data & 8 & 13419 & 7632 & \multirow{7}{*}{$\begin{array}{c}0.54 \\
\pm 0.01\end{array}$} & \multirow{7}{*}{$\begin{array}{c}2.8 \\
\pm 1.7\end{array}$} \\
\hline & $Z+$ jets & $0.14 \pm 0.09$ & $57 \pm 26$ & $180 \pm 49$ & & \\
\hline & $W+$ jets & $2.3 \pm 0.7$ & $140 \pm 51$ & $290 \pm 75$ & & \\
\hline & diboson & $0.40 \pm 0.24$ & $3.1 \pm 1.4$ & $10.7 \pm 3.6$ & & \\
\hline & top & $0.09 \pm 0.05$ & $0.1 \pm 0.1$ & $3.8 \pm 1.9$ & & \\
\hline & multi-jet & $5.1 \pm 2.9$ & $13220 \pm 130$ & $7150 \pm 130$ & & \\
\hline & Ref. point 3 & $1.4 \pm 0.5$ & $0.11 \pm 0.11$ & $1.3 \pm 0.4$ & & \\
\hline
\end{tabular}

Table 4. The MC predicted backgrounds in the multi-jet control regions, including both the statistical and systematic uncertainties, and the expected multi-jet contribution (in italics), obtained by subtracting the MC contributions from observed data (in bold). Predicted event yields for the SUSY reference points in the control regions are also shown. The estimated multi-jet contribution in the SRs is given in the last column. The details of the systematic uncertainties reported here are discussed in section 9 .

the reconstructed invariant mass of the two leptons, $m_{\tau, \mu}$, must be outside the $Z$ mass window $\left(12 \mathrm{GeV}<m_{\tau, \mu}<40 \mathrm{GeV}\right.$ or $\left.m_{\tau, \mu}>100 \mathrm{GeV}\right)$. To further suppress multi-jet and $Z+$ jets events, $E_{\mathrm{T}}^{\text {miss }}>40 \mathrm{GeV}$ is required, and the leptons must not be back-to-back, i.e. $|\Delta \phi(\tau, \mu)|<2.7$ and $|\Delta \eta(\tau, \mu)|<2.0$. The contribution from events with top quarks is suppressed by rejecting events containing $b$-tagged jets, i.e. $N($ B20 $)=0$. The definition of the $W$ CR is given in table 6 .

The multi-jet contribution in the $W \mathrm{CR}$ is estimated by counting the number of events in data satisfying the same requirements as the $W$ CR but with same-sign (SS) charge of 


\begin{tabular}{|c|c|c|c|c|}
\hline Sample & VR-C1N2 & VR-C1C1 & VR-DS-highMass & VR-DS-lowMass \\
\hline Data & 5585 & 1846 & 163 & 764 \\
SM total & $5840 \pm 340$ & $1870 \pm 87$ & $170 \pm 24$ & $860 \pm 110$ \\
\hline Multi-jet & $5370 \pm 320$ & $1570 \pm 61$ & $120 \pm 19$ & $670 \pm 100$ \\
$W+$ jets & $320 \pm 93$ & $240 \pm 60$ & $37 \pm 15$ & $130 \pm 42$ \\
$Z+$ jets & $97 \pm 39$ & $34 \pm 12$ & $5.6 \pm 2.9$ & $36 \pm 14$ \\
Diboson & $27 \pm 8$ & $15.1 \pm 5.4$ & $5.1 \pm 1.5$ & $12.9 \pm 3.6$ \\
Top & $24 \pm 10$ & $5.0 \pm 2.1$ & $3.6 \pm 1.7$ & $9.5 \pm 3.3$ \\
\hline
\end{tabular}

Table 5. Number of events in the multi-jet validation regions $\mathrm{F}$ for data and SM backgrounds, including both statistical and systematic uncertainties. The SM MC backgrounds are normalised to $20.3 \mathrm{fb}^{-1}$. The multi-jet contribution is estimated from data with the ABCD method, by multiplying the number of events in region $\mathrm{E}$ and the transfer factor $\mathrm{T}$.

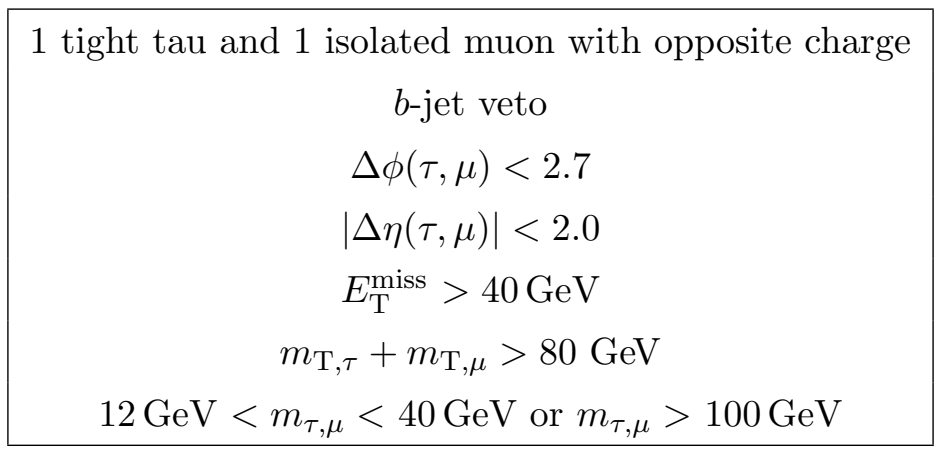

Table 6. Definition of the $W+$ jets control region.

the two leptons. Events from SM processes other than multi-jet production are subtracted from the data counts in the SS region, using their MC prediction. The method relies on the fact that in the multi-jet background the ratio of SS to OS events is close to unity, whilst a significant difference from unity is expected for $W+$ jets production. The latter is dominated by $g u / g d$-initiated processes that often give rise to a jet originating from a quark, the charge of which is anti-correlated with the $W$ boson charge.

The $W+$ jets estimate is tested in a validation region ( $W \mathrm{VR}$ ) where the $W$ is required to decay leptonically into an electron and a neutrino. The $W$ VR is defined in the same way as the $W$ CR except that events with one tight tau and one isolated electron are selected. The multi-jet contribution in the $W$ VR is estimated using the same technique as in the $W \mathrm{CR}$. The event yields in the $W+$ jets control and validation regions are given in table 7 . The purity of the selection in $W$ +jets events is around $80 \%(75 \%)$ in the $W$ CR (VR). Good agreement between data and SM predictions is observed. The large uncertainties stem from the corrections applied for the trigger and identification efficiency for misidentified taus from light jets. Distributions of the kinematic variables defining the SRs are shown in figure 4 . The distribution of the effective mass $m_{\mathrm{eff}}$ in the $W$ VR is shown in figure 5 (a). 


\begin{tabular}{|c|c|c|}
\hline Sample & $W$ CR & $W$ VR \\
\hline Data & 4120 & 3420 \\
\hline SM total & $4100 \pm 900$ & $3500 \pm 600$ \\
\hline$W+$ jets & $3300 \pm 800$ & $2600 \pm 500$ \\
Top & $250 \pm 80$ & $240 \pm 70$ \\
Diboson & $180 \pm 50$ & $170 \pm 40$ \\
$Z+$ jets & $140 \pm 40$ & $99 \pm 31$ \\
Multi-jet & $250 \pm 250$ & $400 \pm 200$ \\
\hline
\end{tabular}

Table 7. Event yields in the $W+$ jets control and validation regions. The SM backgrounds other than multi-jet production are estimated from MC simulation and normalised to $20.3 \mathrm{fb}^{-1}$. The multi-jet contribution is estimated from data in the same-sign region, by subtracting all other SM backgrounds from the data counts. The quoted uncertainty is the sum in quadrature of statistical and systematic uncertainties.

\subsection{Estimation of irreducible backgrounds}

Irreducible SM backgrounds arise mainly from $t \bar{t}$, single top, $t \bar{t}+V, Z / \gamma^{*}+$ jets and diboson ( $W W, W Z$ and $Z Z$ ) processes and are estimated with $\mathrm{MC}$ simulation and validated in data. Other SM backgrounds are negligible.

The diboson background accounts for $15-35 \%$ of the total SM contribution in the signal regions, and mainly arises from $W W \rightarrow \tau \nu \tau \nu$ and $Z Z \rightarrow \tau \tau \nu \nu$ events. To validate the MC modelling and normalisation of the $W W$ process, validation regions with enriched $W W \rightarrow e \nu \mu \nu$ contribution are defined for each SR. Events with exactly one OS electronmuon pair are selected. To keep the same phase space as the SRs, the $W W$ VRs are defined in the same way except for intermediate requirements on the relevant kinematic variable to reduce possible signal contamination, i.e. $50 \mathrm{GeV}<m_{\mathrm{T} 2}<100 \mathrm{GeV}$ for SR$\mathrm{C} 1 \mathrm{~N} 2,150 \mathrm{GeV}<m_{\mathrm{T}, \mu}+m_{\mathrm{T}, e}<250 \mathrm{GeV}$ for SR-C1C1, $150 \mathrm{GeV}<m_{\mathrm{eff}}<230 \mathrm{GeV}$ for SR-DS-highMass and $150 \mathrm{GeV}<m_{\text {eff }}<260 \mathrm{GeV}$ for SR-DS-lowMass. The purity of the selection in $W W$ events ranges from $70 \%$ to $80 \%$ in all validation regions. The agreement between data and SM predictions is found to be good, and the data yields match the SM predictions within uncertainties (of the order of 10\%), as shown in figure 5 (c).

The inclusive contribution from $t \bar{t}$, single top, $t \bar{t}+V$ and $Z / \gamma^{*}+$ jets amounts to about $5-20 \%$ of the total background in the signal regions. The MC estimates are validated in regions enriched in $Z+$ jets and $t \bar{t}$ events.

In the $Z+$ jets validation region events containing one isolated electron or muon and one tau with opposite electrical charge are selected. The invariant mass of the dilepton pair must satisfy $40 \mathrm{GeV}<m_{\ell \tau}<75 \mathrm{GeV}$. To suppress contributions from other SM processes, $m_{\mathrm{T}, l}+m_{\mathrm{T}, \tau}<80 \mathrm{GeV},|\Delta \phi(\ell, \tau)|>2.4$ and $N(\mathrm{~B} 20)=0$ veto are required. The multi-jet contribution in the $Z$ VR is estimated with the same method as in the $W$ CR and VR.

The top quark validation region is defined by requiring at least two candidate taus, and at least one must satisfy the tight jet BDT quality requirement. At least one of the selected 


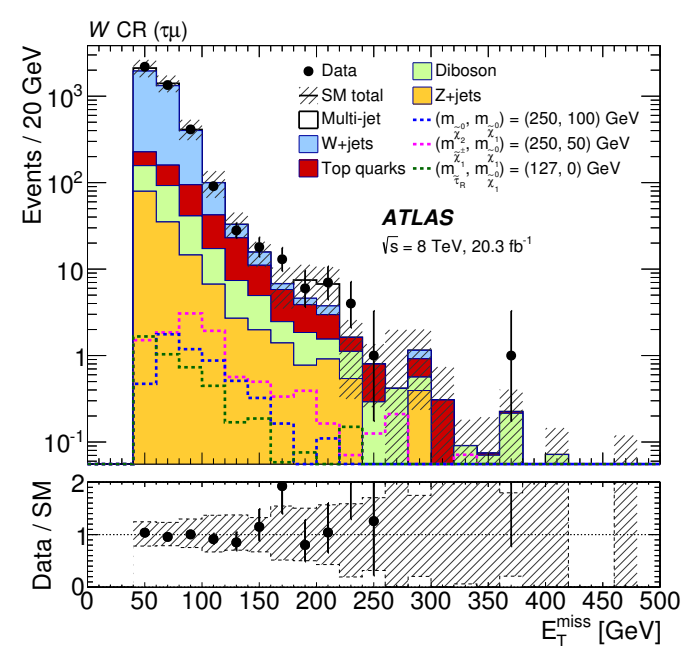

(a)

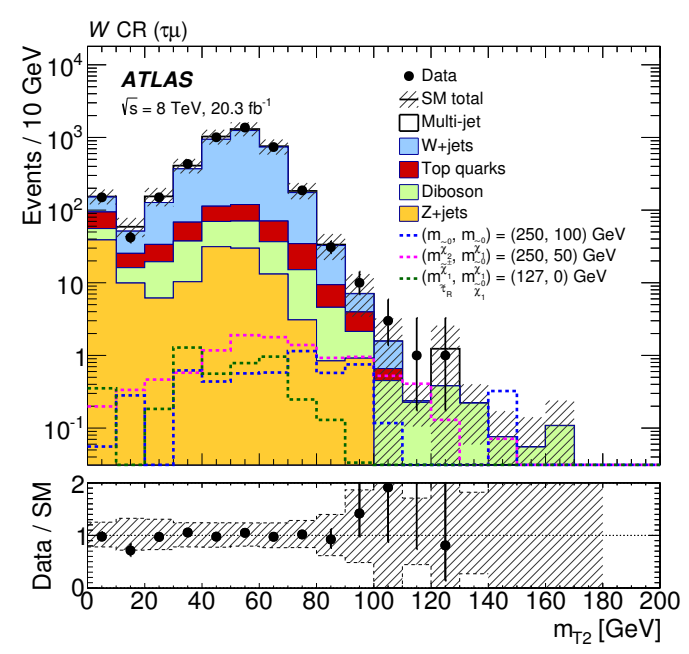

(c)
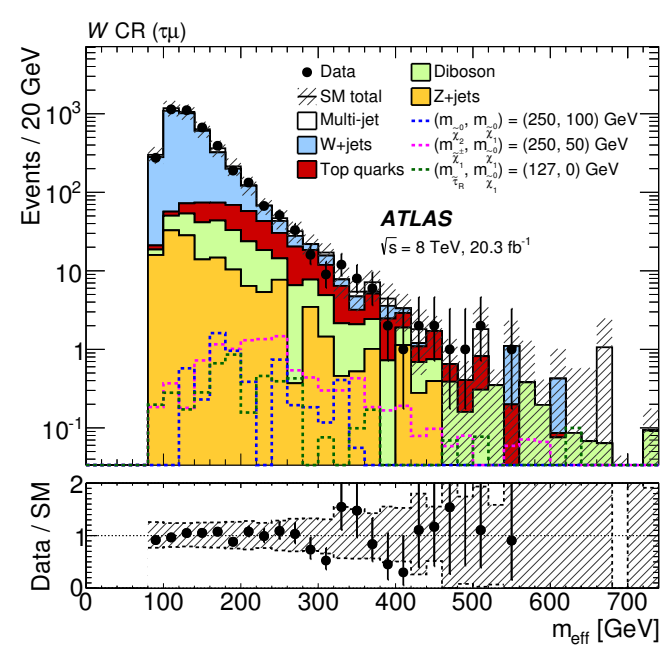

(b)

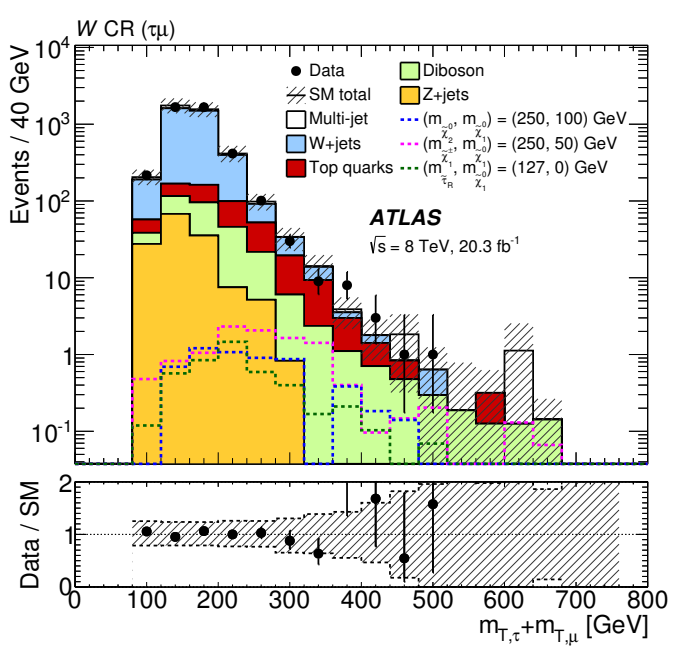

(d)

Figure 4. Distributions in the $W+$ jets control region of the kinematic variables which are used in the signal region definition. The SM backgrounds other than multi-jet production are estimated from MC simulation and normalised to $20.3 \mathrm{fb}^{-1}$. The multi-jet contribution is estimated from data in the same-sign region, by subtracting all other SM backgrounds from the data counts. The hatched bands represent the combined statistical and systematic uncertainties on the total SM background. For illustration, the distributions of the SUSY reference points are also shown as dashed lines. The lower panels show the ratio of data to the SM background estimate.

tau pairs must contain taus with opposite electrical charge. To increase the contribution from top events, two $b$-tagged jets with $p_{\mathrm{T}}>20 \mathrm{GeV}$ are required, and the events should be kinematically compatible with $t \bar{t}$ production (top-tagged) through the use of the variable $m_{\mathrm{CT}}$ [97]. The scalar sum of the $p_{\mathrm{T}}$ of the two taus and of at least one combination of two jets in an event must exceed $100 \mathrm{GeV}$. Furthermore, top-tagged events are required to possess $m_{\mathrm{CT}}$ values calculated from combinations of jets and taus consistent with the expected bounds from $t \bar{t}$ events as described in ref. [98]. Requirements of $50 \mathrm{GeV}<E_{\mathrm{T}}^{\text {miss }}<100 \mathrm{GeV}$ 


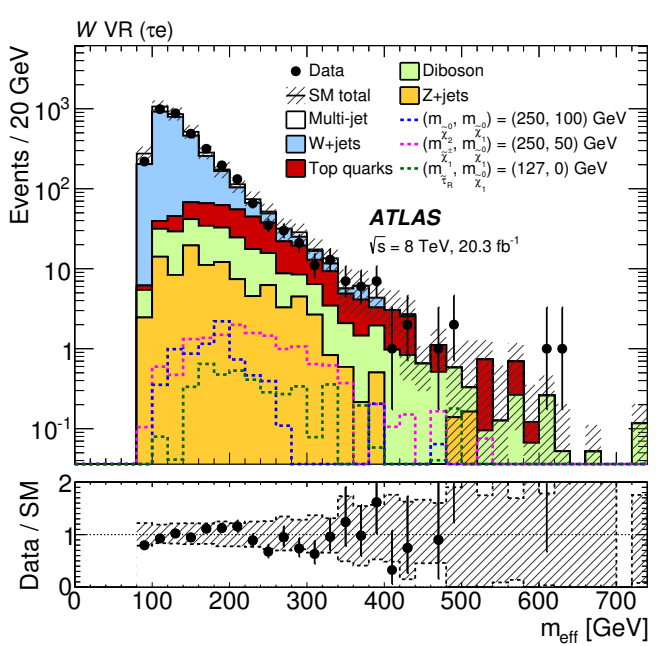

(a) $W$ VR

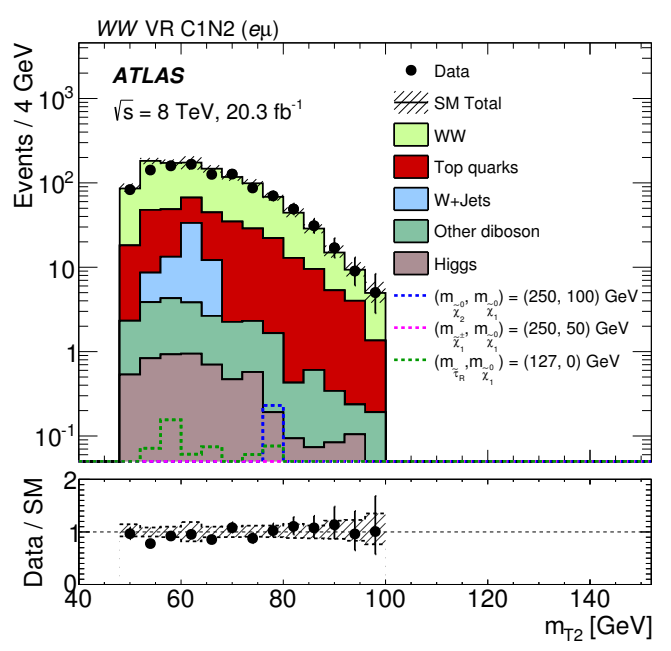

(c) $W W$ VR C1N2
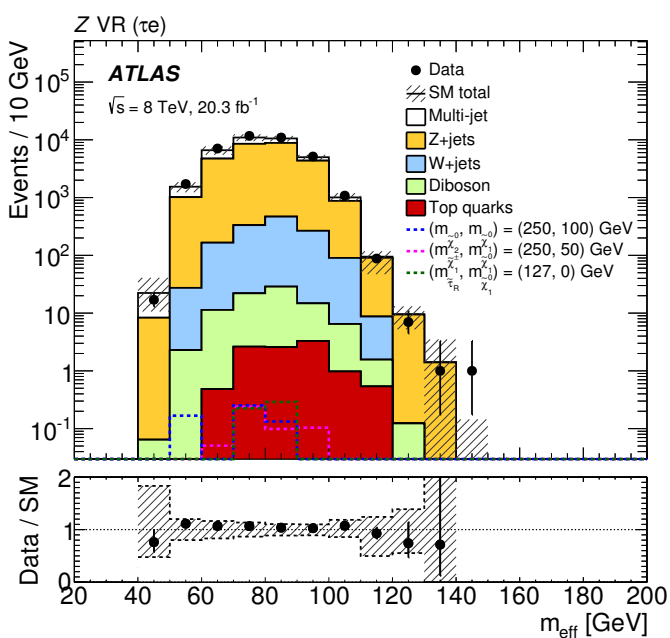

(b) $Z \mathrm{VR}$
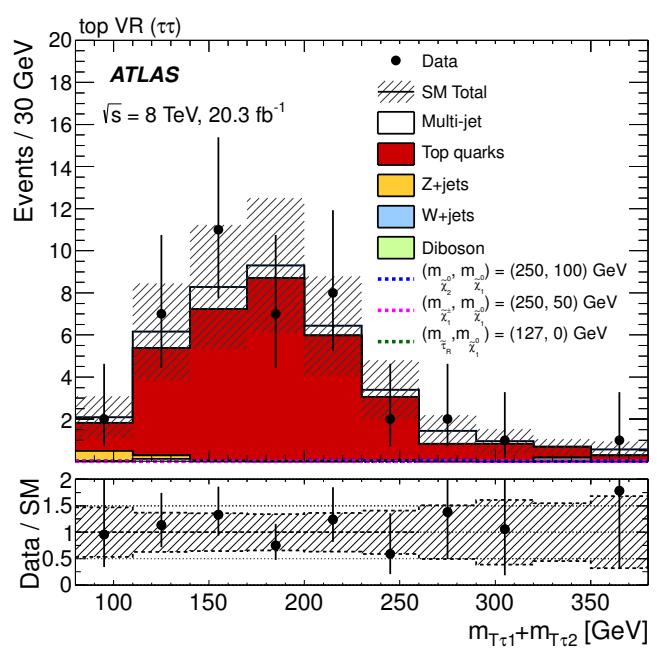

(d) top VR

Figure 5. Distributions of relevant kinematic variables in the validation regions: $m_{\mathrm{eff}}$ in the (a) $W$ and (b) $Z$ VRs, (c) $m_{\mathrm{T} 2}$ in the $W W$ VR C1N2, and (d) $m_{\mathrm{T} \tau 1}+m_{\mathrm{T} \tau 2}$ in the top VR. In the $Z \mathrm{VR}$, events containing one electron and one tau are shown. The SM backgrounds other than multi-jet production are estimated from MC simulation and normalised to $20.3 \mathrm{fb}^{-1}$. The hatched bands represent the combined statistical and systematic uncertainties on the total SM background. For illustration, the distributions of the SUSY reference points are also shown as dashed lines. The lower panels show the ratio of data to the SM background estimate.

and $m_{\mathrm{T} \tau 1}+m_{\mathrm{T} \tau 2}>80 \mathrm{GeV}$ are applied to suppress contributions from SM backgrounds not containing top quarks.

The purity of the selection in $Z+$ jets and $t \bar{t}$ events is above $80 \%$ in the respective validation regions and good agreement between the data and SM expectation is observed. Distributions of relevant kinematic variables in the $Z$ and top VRs are shown in figure 5 (b) and (d). 


\subsection{Fitting procedure}

For each signal region, a simultaneous fit is performed based on the profile likelihood method [96]. The inputs to the fit are: the number of observed events in the multi-jet CR A and $W$ CR, the expected non-multi-jet and non- $W$ contributions to the multi-jet CR A and $W$ CR, the expected contributions of multi-jet and $W$ to the multi-jet CR A and $W \mathrm{CR}$, as described in sections 8.1-8.2, and the transfer factors, which relate the number of multi-jet or $W+$ jets events in their associated control region to that predicted in the signal region. The number of events in a given $\mathrm{CR}$ is described using a Poisson distribution, the mean of which is the sum of the expected contributions from all background sources. The free parameters in the fit are the normalisations of the $W+$ jets and multi-jet contributions. The systematic uncertainties on the expected background yields are included as nuisance parameters, assumed to be Gaussian distributed with a width determined from the size of the uncertainty. Correlations between control and signal regions, and background processes are taken into account with common nuisance parameters. The fit parameters are determined by maximising the product of the Poisson probability functions and the constraints for the nuisance parameters.

\section{$9 \quad$ Systematic uncertainties}

Systematic uncertainties have an impact on the estimates of the background and signal event yields in the control and signal regions. Several sources of systematic uncertainty are considered for the ABCD method used to determine the multi-jet background: the correlation between the kinematic variable $\left(m_{\mathrm{T} 2}, m_{\mathrm{T} \tau 1}+m_{\mathrm{T} \tau 2}\right.$, or $\left.m_{\mathrm{eff}}\right)$ and the tau identification variable, the limited number of events in the CRs, and the subtraction of other SM backgrounds. The systematic uncertainty on the correlation is estimated by comparing the transfer factor from the region $\mathrm{B}$ to region $\mathrm{C}$ to that of the region $\mathrm{E}$ to $\mathrm{F}$ (see figure 2). The systematic uncertainty due to the limited number of events in the control regions is estimated by considering the statistical uncertainty on the number of data events and the other SM background components. The systematic uncertainty on the non-multi-jet background subtraction in the control regions $\mathrm{A}, \mathrm{B}$, and $\mathrm{C}$ is estimated by considering the systematic uncertainty in the MC estimations of the non-multi-jet background in the CRs. A summary of the systematic uncertainties on the multi-jet background estimation in the SRs is shown in table 8 . The dominant source is given by the limited number of events in the multi-jet control regions.

The dominating uncertainty in the multi-jet estimation from SS events in the $W$ control region and in the $W$ and $Z$ validation regions stems from the different fractions of quark and gluon jets in the OS and SS regions. The relative difference in the tau mis-identification rates measured in events with OS and SS lepton pairs varies between $20 \%$ and $70 \%$ in most of the phase space of interest for this analysis; however, it goes up to $100 \%$ in regions with few events.

The experimental systematic uncertainties on the SM backgrounds estimated with MC simulation are due to those on the jet energy scale and resolution [85], $E_{\mathrm{T}}^{\text {miss }}$ energy scale and resolution [91], b-tagging and mis-identification efficiency [99], and tau identification, 


\begin{tabular}{|c|c|c|c|c|}
\hline Systematic Source & SR-C1N2 & SR-C1C1 & SR-DS-highMass & SR-DS-lowMass \\
\hline Correlation & $4.9 \%$ & $1.6 \%$ & $8.0 \%$ & $14 \%$ \\
Non-multi-jet subtraction in Region A & $8.0 \%$ & $12 \%$ & $21 \%$ & $13 \%$ \\
Non-multi-jet subtraction in Region B & $1.0 \%$ & $0.4 \%$ & $1.2 \%$ & $0.5 \%$ \\
Non-multi-jet subtraction in Region C & $2.7 \%$ & $1.4 \%$ & $3.6 \%$ & $2.0 \%$ \\
Number of events in Region A & $61 \%$ & $38 \%$ & $133 \%$ & $57 \%$ \\
Number of events in Regions C and B & $1.0 \%$ & $2.0 \%$ & $8.4 \%$ & $1.5 \%$ \\
\hline Total & $62 \%$ & $40 \%$ & $135 \%$ & $60 \%$ \\
\hline
\end{tabular}

Table 8. Summary of the systematic uncertainties for the multi-jet background estimation. The total uncertainty is the sum in quadrature of each source.

trigger efficiency and energy scale [89]. The main contribution comes from the uncertainty on the corrections applied to the tau identification and trigger efficiency in the simulated samples, and from the tau energy scale uncertainty. The uncertainty on the correction for the tau identification efficiency is of the order of $2-3 \%$ for taus with $p_{\mathrm{T}}$ between 20 and $200 \mathrm{GeV}$. Uncertainties on the trigger efficiency are around $2-4 \%$ for taus with $p_{\mathrm{T}}$ between 30 and $50 \mathrm{GeV}$, and increase to $8-10 \%$ for taus with $p_{\mathrm{T}}>50 \mathrm{GeV}$. The uncertainty on the tau energy scale is of the order of a few percent, with no significant dependence on pile-up or on the tau pseudorapidity.

A systematic uncertainty of the order of $10 \%$ associated with the simulation of pile-up is also taken into account. The luminosity uncertainty is $2.8 \%$ [51].

Theoretical uncertainties affecting the generator predictions arise from the finite number of partons (FNP) from the hard primary interaction for diboson and top processes, the effect of the renormalisation $\left(\mu_{\mathrm{R}}\right)$ and factorisation $\left(\mu_{\mathrm{F}}\right)$ scales for diboson, $W+$ jets and $Z+$ jets production, and the impact of the merging scale of the matrix element with the parton shower (ME-PS) for diboson and top processes. The FNP-induced uncertainty is calculated by comparing the baseline samples which contain up to three additional partons from the hard interaction to samples with up to two additional partons. The uncertainty due to the $\mu_{\mathrm{R}}$ and $\mu_{\mathrm{F}}$ scales is determined by the comparison with samples with these scales varied up and down by a factor of two, while the ME-PS merging scale uncertainty is determined by a comparison with samples with varied merging scale. For $W+$ jets events, the uncertainty due to the jet $p_{\mathrm{T}}$ threshold used for parton-jet matching is calculated by comparing the baseline samples with jet $p_{\mathrm{T}}$ threshold set to $15 \mathrm{GeV}$ to samples with a threshold of $25 \mathrm{GeV}$. The theoretical uncertainties associated with the top sample vary from $10 \%$ to $20 \%$ while the $W / Z$ +jets and diboson theoretical uncertainties vary from $17 \%$ to $30 \%$, depending on the process and the signal region. The uncertainty due to the PDF choice is $1-2 \%$ for $W / Z+$ jets and diboson production, and around $6 \%$ for $t \bar{t}$, single top and $t \bar{t}+V$ processes. The theoretical uncertainties on the cross sections are $5 \%$ for $Z+$ jets [100], $10 \%$ for $t \bar{t}, 3 \%, 4 \%$ and $7 \%$ for single top in the $t$-, $s$ - and $W t$ - channels, respectively, $22 \%$ for $t \bar{t}+W / Z$, and $5 \%, 5 \%$ and $7 \%$ for $W W, Z Z$ and $W Z$, respectively [74].

The various sources of uncertainty for the non-multi-jet background estimates in the SRs are summarised in table 9 . In the $W$ CR, the total uncertainty on the $W+$ jets 


\begin{tabular}{|c|c|c|c|c|}
\hline Source & $W+$ jets & Diboson & $Z+$ jets & Top \\
\hline MC statistics & $16-36 \%$ & $15-28 \%$ & $44-80 \%$ & $23-50 \%$ \\
Theoretical uncertainty & $17-30 \%$ & $17-27 \%$ & $25-30 \%$ & $10-20 \%$ \\
Tau ID and trigger & $10-18 \%$ & $20-21 \%$ & $10-20 \%$ & $22-28 \%$ \\
Tau Energy Scale & $12-20 \%$ & $3-13 \%$ & $4-12 \%$ & $2-7 \%$ \\
Others & $1-10 \%$ & $3-9 \%$ & $5-10 \%$ & $10-20 \%$ \\
\hline Total & $34-48 \%$ & $35-44 \%$ & $58-85 \%$ & $43-62 \%$ \\
\hline
\end{tabular}

Table 9. Summary of the various sources of uncertainty for the non-multi-jet background estimates in the signal regions. The first row shows the uncertainty due to the limited statistics in the MC samples. The second row shows the total systematic uncertainty from theory. The main experimental systematic uncertainties are given in the rows labelled with "Tau ID and trigger" and "Tau Energy scale", while the row "Others" shows the contribution from the remaining sources of experimental systematic uncertainty as described in the text. The "Total" uncertainty is the sum in quadrature of each source.

estimation is around $20 \%$, and it is dominated by theory uncertainties and corrections due to trigger and tau identification efficiency.

Signal cross sections are calculated to NLO. Their uncertainties are taken from an envelope of cross-section predictions using different PDF sets and factorisation and renormalisation scales [101]. Uncertainties associated with modelling of ISR in SUSY signal samples were evaluated for a few benchmark samples and found to be negligible. Systematic uncertainties associated with the signal selection efficiency include those due to the tau trigger efficiency, tau identification and energy scale, jet reconstruction and $E_{\mathrm{T}}^{\mathrm{miss}}$ calculation. The uncertainty on the integrated luminosity also affects the predicted signal yield. The total uncertainty varies between $20 \%$ and $30 \%$ for SUSY scenarios to which this measurement is sensitive. The dominant experimental uncertainty is due to the tau trigger efficiency, and is around $20 \%$.

\section{Results}

The observed number of events in each signal region and the expected contributions from SM processes are given in table 10. The contributions of multi-jet and $W+$ jets events were scaled with the normalisation factors obtained from the fit described in section 8.4. The normalisation factors do not deviate from unity by more than $1 \%$. Due to the limited number of events in the multi-jet CRs, the uncertainty on the multi-jet normalisation varies between $54 \%$ and $131 \%$ depending on the CR, while the uncertainty on the $W+$ jets normalisation is around 10\%. All statistical uncertainties arising from the limited number of MC events are included. The effect of limited data events in the CRs is included in the systematic uncertainty. All systematic uncertainties except for the jet energy scale and jet energy resolution are obtained taking into account the correlations among control regions and background processes. The total uncertainties are the sum in quadrature of 
systematic and statistical uncertainties. Due to the simultaneous fit, the uncertainty on the $W+$ jets estimation decreases by $10 \%$ with respect to the numbers in table 9 , while the uncertainties on the remaining non-multi-jet backgrounds vary by less than $2 \%$ depending on the SR. Due to the large uncertainty on the multi-jet normalisation, the uncertainty on the multi-jet estimate increases by up to $25 \%$ depending on the SR with respect to the numbers quoted in table 4. Agreement is found between observations and background expectations within $1 \sigma$ in all signal regions except for SR-C1C1. In SR-C1C1, the observed number of events fluctuates below the number of expected SM events by $2.2 \sigma$, mainly in the region $250 \mathrm{GeV}<m_{\mathrm{T} \tau 1}+m_{\mathrm{T} \tau 2}<270 \mathrm{GeV}$.

For each SR, the significance of a possible excess of observed events over the SM prediction is quantified by the one-sided probability, $p_{0}$, of the background alone to fluctuate to the observed number of events or higher using the asymptotic formula described in [96]. A fit similar to the one described in section 8.4 is used, except that the number of events observed in the SR is added as an input to the fit, and an additional parameter for the non-SM signal strength, constrained to be non-negative, is fit. Upper limits at 95\% confidence level (CL) on the number of non-SM events in the SRs are derived using the CLs prescription [102] and neglecting any possible signal contamination in the control regions. Normalising these by the integrated luminosity of the data sample, they can be interpreted as upper limits on the visible non-SM cross section, $\sigma_{\text {vis }}^{95}$, which is defined as the product of acceptance, reconstruction efficiency and production cross section. All systematic uncertainties and their correlations are taken into account via nuisance parameters. The accuracy of the limits obtained by the asymptotic formula was tested for all SRs by randomly generating a large number of pseudo-datasets and repeating the fit, and good agreement was found. The results are given in table 10 .

In figure 6 the distributions of the relevant kinematic variables are shown for data, SM expectations and illustrative SUSY benchmark models. The SM background distributions are taken from MC simulation, except for the multi-jet contribution, which is estimated using the ABCD method described in section 8.1. The normalisation factors obtained from the fit detailed in section 8.4 are used to correct the expected distributions of the $W+$ jets and multi-jet processes.

\section{Interpretation}

In the absence of a significant excess over the SM background expectations, the observed numbers of events in the signal regions are used to place model-dependent exclusion limits at $95 \%$ CL for the pMSSM and the simplified models described in section 5.2. The same CLs limit-setting procedure as described in section 10 is used, except that the SUSY signal is allowed to populate both the signal and the control regions. All SRs defined in section 7 are considered in order to derive limits for all the SUSY models considered in this paper, regardless of the specific production mode for which they were optimised. Since the SRs are not mutually exclusive, for each point in the parameter space the SR which gives the best expected limit is used. 


\begin{tabular}{|c|c|c|c|c|}
\hline SM process & SR-C1N2 & SR-C1C1 & SR-DS-highMass & SR-DS-lowMass \\
\hline Top & $0.30 \pm 0.19$ & $0.7 \pm 0.4$ & $0.9 \pm 0.4$ & $1.3 \pm 0.6$ \\
\hline$Z+$ jets & $0.9 \pm 0.5$ & $0.20 \pm 0.17$ & $0.6 \pm 0.4$ & $0.40 \pm 0.27$ \\
\hline$W+$ jets & $2.2 \pm 0.8$ & $11.2 \pm 2.8$ & $2.7 \pm 0.9$ & $4.1 \pm 1.2$ \\
\hline Diboson & $2.2 \pm 0.9$ & $3.8 \pm 1.4$ & $2.5 \pm 1.0$ & $2.9 \pm 1.0$ \\
\hline Multi-jet & $2.3 \pm 2.0$ & $5.8 \pm 3.3$ & $0.9 \pm 1.2$ & $2.8 \pm 2.3$ \\
\hline SM total & $7.9 \pm 2.4$ & $22 \pm 5$ & $7.5 \pm 1.9$ & $11.5 \pm 2.9$ \\
\hline Observed & 11 & 12 & 7 & 15 \\
\hline Ref. point 1 & $11.3 \pm 2.8$ & $8.5 \pm 2.2$ & $10.2 \pm 2.6$ & $7.5 \pm 2.0$ \\
\hline Ref. point 2 & $9.2 \pm 2.1$ & $20 \pm 4$ & $12.4 \pm 2.8$ & $12.8 \pm 2.7$ \\
\hline Ref. point 3 & $0.8 \pm 0.5$ & $7.6 \pm 1.9$ & $3.8 \pm 1.0$ & $5.2 \pm 1.3$ \\
\hline$p_{0}$ & 0.20 & 0.50 & 0.50 & 0.21 \\
\hline Expected $\sigma_{\text {vis }}^{95}(\mathrm{fb})$ & $<0.42_{-0.11}^{+0.19}$ & $<0.56_{-0.14}^{+0.25}$ & $<0.37_{-0.10}^{+0.17}$ & $<0.51_{-0.15}^{+0.18}$ \\
\hline Observed $\sigma_{\text {vis }}^{95}(\mathrm{fb})$ & $<0.59$ & $<0.37$ & $<0.37$ & $<0.66$ \\
\hline
\end{tabular}

Table 10. Observed and expected numbers of events in the signal regions for $20.3 \mathrm{fb}^{-1}$. The contributions of multi-jet and $W+$ jets events were scaled with the normalisation factors obtained from the fit described in section 8.4. The shown uncertainties are the sum in quadrature of statistical and systematic uncertainties. The correlation of systematic uncertainties among control regions and background processes is fully taken into account and, as a result, the numbers given here may be different from those in tables 4 and 9. Expected event yields for the SUSY reference points (see section 5.2) are also shown. The one-sided $p_{0}$-values and the observed and expected $95 \%$ CL upper limits on the visible non-SM cross section $\left(\sigma_{\text {vis }}^{95}\right)$, obtained from the fit described in section 10, are given. Values of $p_{0}>0.5$ are truncated to $p_{0}=0.5$.

The results are shown in figures 7-9. The solid (dashed) lines show the observed (expected) exclusion contours. The band around the expected limit shows the $\pm 1 \sigma$ variations, including all uncertainties except theoretical uncertainties on the signal cross section. The dotted lines around the observed limit indicate the sensitivity to $\pm 1 \sigma$ variations of the theoretical uncertainties. All mass limits hereafter quoted correspond to the observed limits reduced by $1 \sigma$ of the signal cross sections.

\subsection{Simplified models: chargino-neutralino and chargino-chargino produc- tion}

The exclusion limits for the simplified models characterised by $\tilde{\chi}_{1}^{ \pm} \tilde{\chi}_{2}^{0}$ and $\tilde{\chi}_{1}^{ \pm} \tilde{\chi}_{1}^{\mp}$ production with intermediate staus are shown in figure 7 . In figure $7(\mathrm{a})$, both production processes are considered simultaneously, whereas in figure $7(\mathrm{~b})$ only chargino-chargino production is assumed.

Chargino masses up to $345 \mathrm{GeV}$ are excluded for a massless lightest neutralino in the scenario of direct production of chargino pairs. In the case of associated production of mass-degenerate charginos and next-to-lightest neutralinos, chargino masses up to $410 \mathrm{GeV}$ 


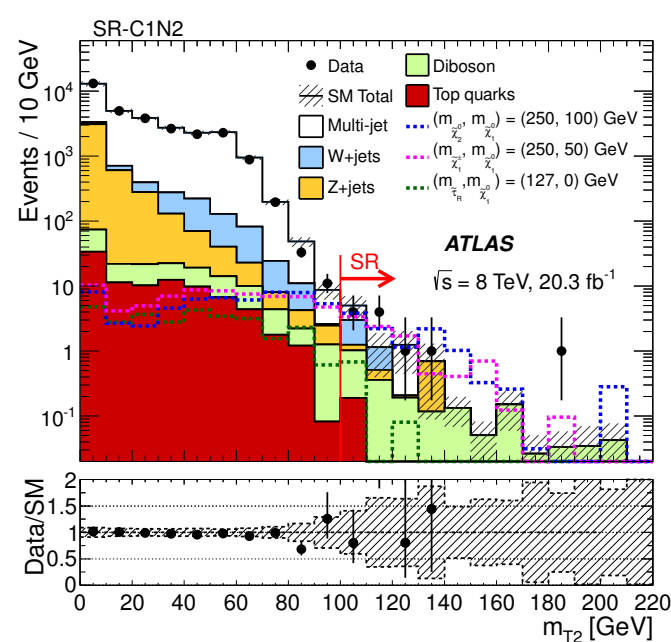

(a) SR-C1N2

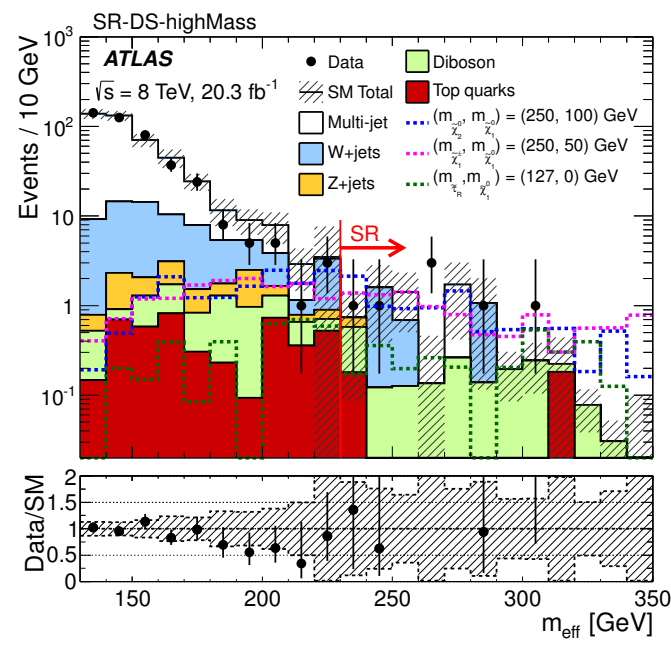

(c) SR-DS-highMass

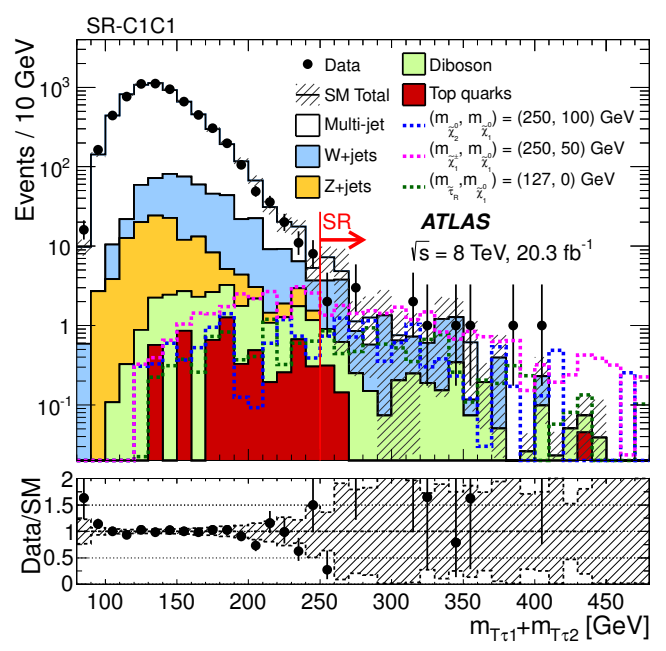

(b) $\mathrm{SR}-\mathrm{C} 1 \mathrm{C} 1$

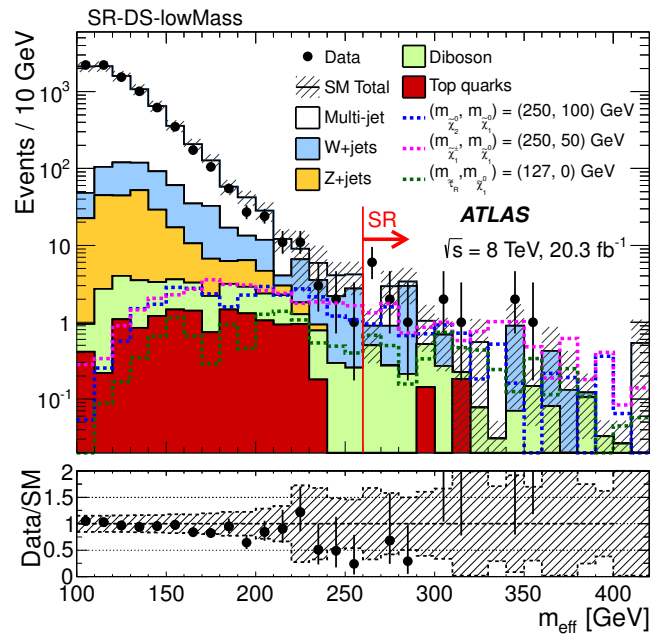

(d) SR-DS-lowMass

Figure 6. Distributions of relevant kinematic variables before the requirement on the given variable is applied: (a) $m_{\mathrm{T} 2}$ for SR-C1N2, (b) $m_{\mathrm{T} \tau 1}+m_{\mathrm{T} \tau 2}$ for SR-C1C1, (c) $m_{\mathrm{eff}}$ for SR-DS-highMass, and (d) $m_{\text {eff }}$ for SR-DS-lowMass. The stacked histograms show the expected SM backgrounds normalised to $20.3 \mathrm{fb}^{-1}$. The multi-jet contribution is estimated from data using the ABCD method. The hatched bands represent the sum in quadrature of systematic and statistical uncertainties on the total SM background. The lower panels show the ratio of data to the total SM background estimate.

are excluded for a massless lightest neutralino. These limits improve the results from the ATLAS three-lepton analysis [37], where only production of $\tilde{\chi}_{1}^{ \pm} \tilde{\chi}_{2}^{0}$ was considered due to the low sensitivity to the decays of $\tilde{\chi}_{1}^{ \pm} \tilde{\chi}_{1}^{\mp}$. For the scenario of direct production of chargino pairs, SR-C1C1 (SR-DS-highMass) provides the best exclusion limit for low (high) chargino masses, while for the associated production of mass-degenerate charginos and next-tolightest neutralinos, SR-C1N2 and SR-DS-highMass have the highest sensitivity over the whole parameter space. 


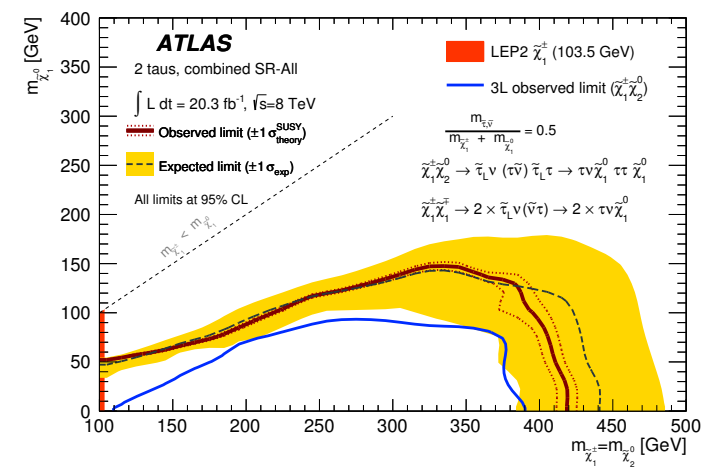

(a) $\tilde{\chi}_{1}^{ \pm} \tilde{\chi}_{1}^{\mp}$ and $\tilde{\chi}_{1}^{ \pm} \tilde{\chi}_{2}^{0}$ production

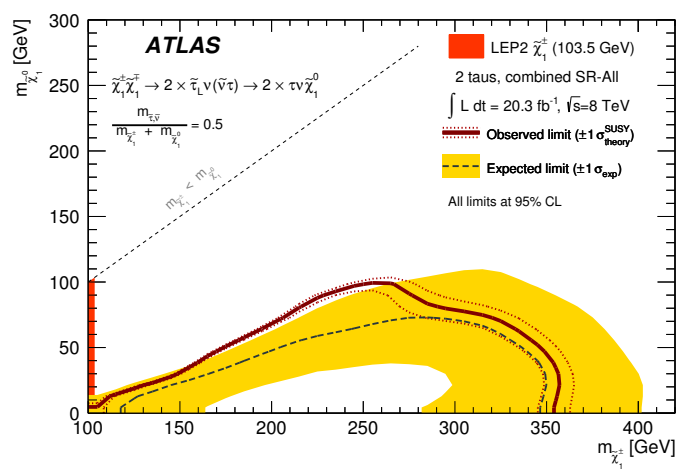

(b) $\tilde{\chi}_{1}^{ \pm} \tilde{\chi}_{1}^{\mp}$ production

Figure 7. 95\% CL exclusion limits for simplified models with (a) a combination of charginoneutralino and chargino-chargino production and (b) chargino-chargino production only. See text for details of exclusion curves and uncertainty bands. Also shown is the LEP limit [39, 40] on the mass of the chargino. The blue contour in (a) corresponds to the observed limit from the ATLAS three-lepton analysis [37], where only $\tilde{\chi}_{1}^{ \pm} \tilde{\chi}_{2}^{0}$ production was considered.

\subsection{Direct stau production}

Due to the low cross section, the sensitivity of this analysis to the direct production of stau pairs is degraded relative to the sensitivity obtained for models of electroweak production of charginos and neutralinos. Upper limits on the cross section were derived for the direct stau production model described in section 5.2, and are shown separately for the production of $\tilde{\tau}_{\mathrm{R}} \tilde{\tau}_{\mathrm{R}}$ and $\tilde{\tau}_{\mathrm{L}} \tilde{\tau}_{\mathrm{L}}$ in figure 8 . For large stau masses, SR-DS-highMass provides the best upper limits, while SR-C1C1 has the best performance for low stau masses. The results in SR-C1C1 lead to a stronger observed exclusion limit than expected due to the number of observed events being fewer than the SM prediction.

The signal strength is defined as the scaling factor that should be applied to the theoretical cross section to exclude the considered model at 95\% CL. The upper limit on the signal strength for the associated production of $\tilde{\tau}_{\mathrm{R}} \tilde{\tau}_{\mathrm{R}}$ and $\tilde{\tau}_{\mathrm{L}} \tilde{\tau}_{\mathrm{L}}$, for different lightest neutralino masses and as a function of the $\tilde{\tau}_{\mathrm{R}}$ mass is shown in figure 9 .

The best observed upper limit on the signal strength is found for a mass of the $\tilde{\tau}_{\mathrm{R}}\left(\tilde{\tau}_{\mathrm{L}}\right)$ of 90.6 (93.1) GeV and a massless $\tilde{\chi}_{1}^{0}$. For this combination of stau and LSP masses, the theoretical cross section at NLO is $0.07(0.17)$ pb for $\tilde{\tau}_{R} \tilde{\tau}_{R}\left(\tilde{\tau}_{L} \tilde{\tau}_{L}\right)$ production, while the excluded cross section is $0.22(0.28) \mathrm{pb}$ and the upper limit on the signal strength for the combined production of $\tilde{\tau}_{\mathrm{R}} \tilde{\tau}_{\mathrm{R}}$ and $\tilde{\tau}_{\mathrm{L}} \tilde{\tau}_{\mathrm{L}}$ is 0.95 .

\subsection{The pMSSM model}

Limits on the mass parameters $M_{2}$ and $\mu$ are set within the pMSSM framework with parameters described in section 5.2. In figure 10(a), the exclusion limits for the pMSSM model with fixed stau mass are shown in the $\mu-M_{2}$ plane. The region at low $M_{2}$ cannot be excluded since it corresponds to points in the parameter space where the chargino and neutralino are lighter than the stau. Since in this model the cross section for direct stau 


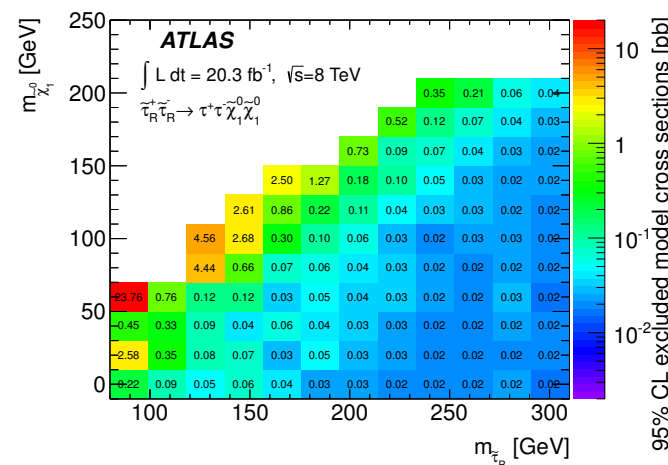

(a)

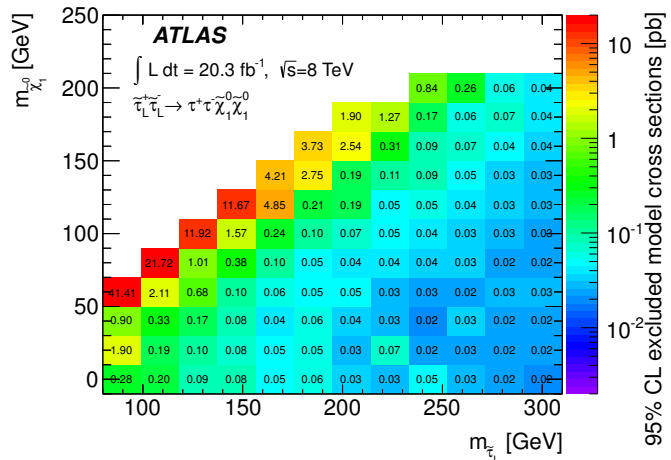

(b)

Figure 8. Upper limits on the cross section for production of only (a) $\tilde{\tau}_{\mathrm{R}} \tilde{\tau}_{\mathrm{R}}$ or $(\mathrm{b}) \tilde{\tau}_{\mathrm{L}} \tilde{\tau}_{\mathrm{L}}$ pairs.
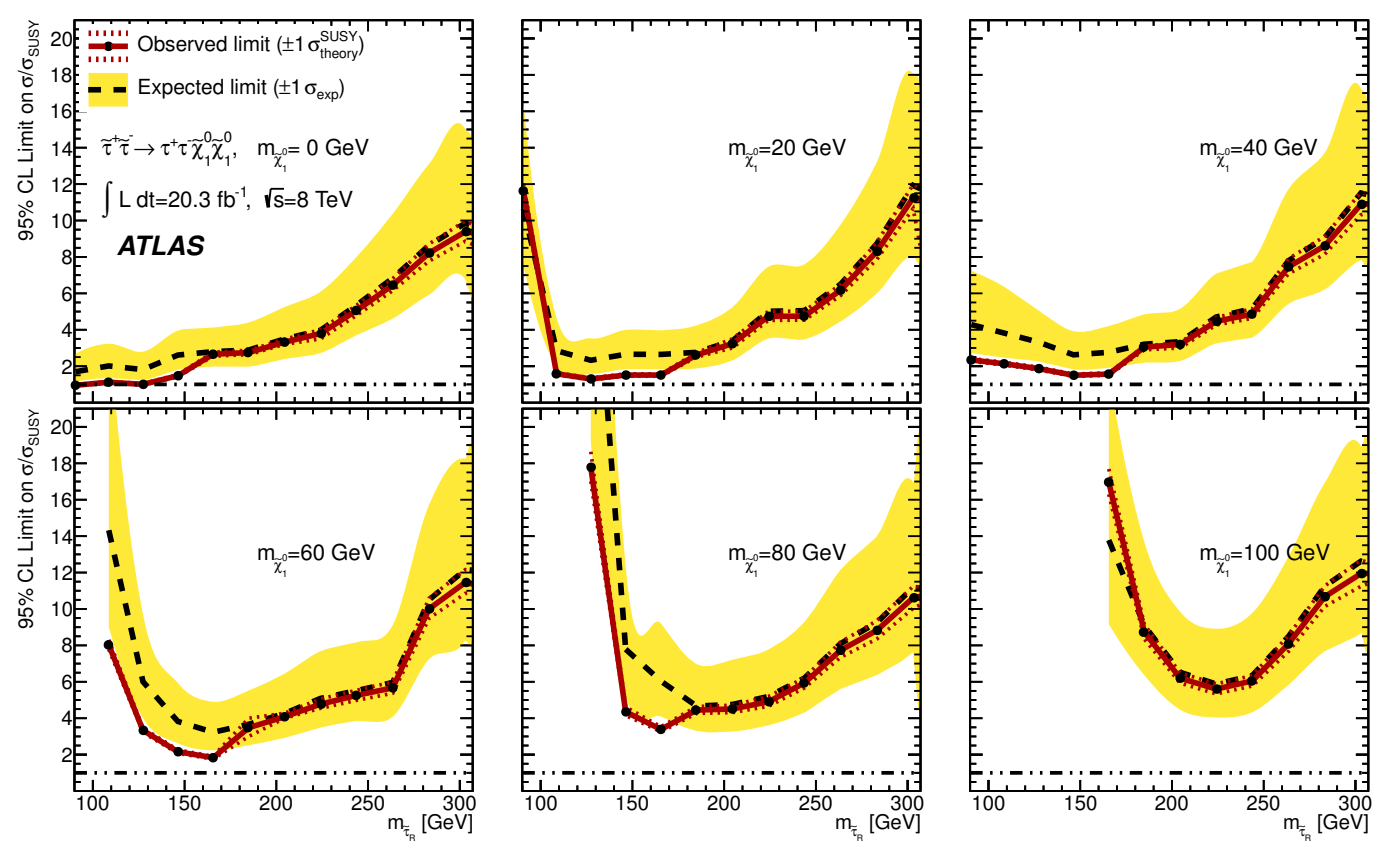

Figure 9. Upper limit on the signal strength for the associated production of $\tilde{\tau}_{\mathrm{R}} \tilde{\tau}_{\mathrm{R}}$ and $\tilde{\tau}_{\mathrm{L}} \tilde{\tau}_{\mathrm{L}}$, for different lightest neutralino masses and as a function of the $\tilde{\tau}_{\mathrm{R}}$ mass. See text for details of exclusion curves and uncertainty bands.

production is constant, this process dominates in the remaining allowed region at large $M_{2}$ and $\mu$. Direct stau production accounts for $60 \%$ of the events for $M_{2}=\mu=400 \mathrm{GeV}$, and $85 \%$ of the events for $M_{2}=\mu=500 \mathrm{GeV}$.

Figure 10(b) shows the exclusion limits in the $\mu-M_{2}$ plane for the pMSSM model with variable stau mass. For both pMSSM models, the excluded $\tilde{\chi}_{1}^{ \pm}\left(\tilde{\chi}_{2}^{0}\right)$ mass range is $100-$ $350 \mathrm{GeV}$ as can be seen from the light grey iso-mass lines of $\tilde{\chi}_{1}^{ \pm}\left(\tilde{\chi}_{2}^{0}\right)$. For values of $\mu$ larger than those simulated for this analysis, the pMSSM phenomenology is similar to that studied here. For larger values of $M_{2}$, the production cross section of heavier neutralinos and charginos increases. In general, the shown limits on the lightest chargino mass can 


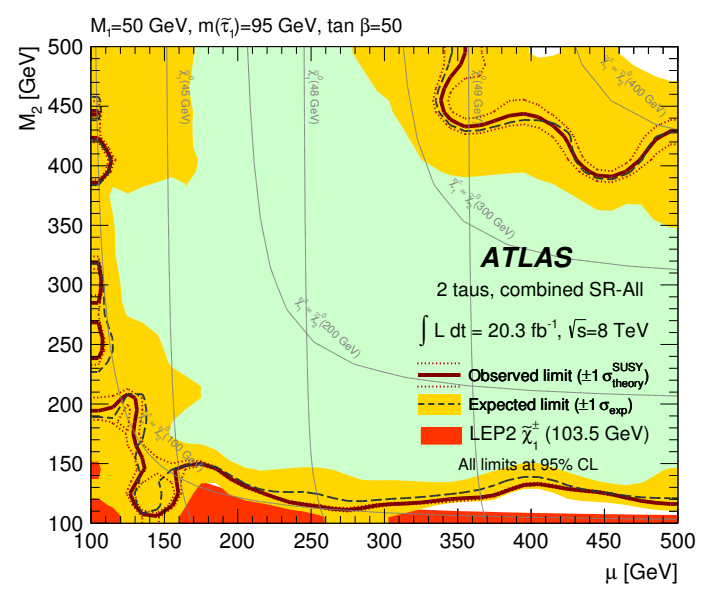

(a) pMSSM model (fixed stau mass)

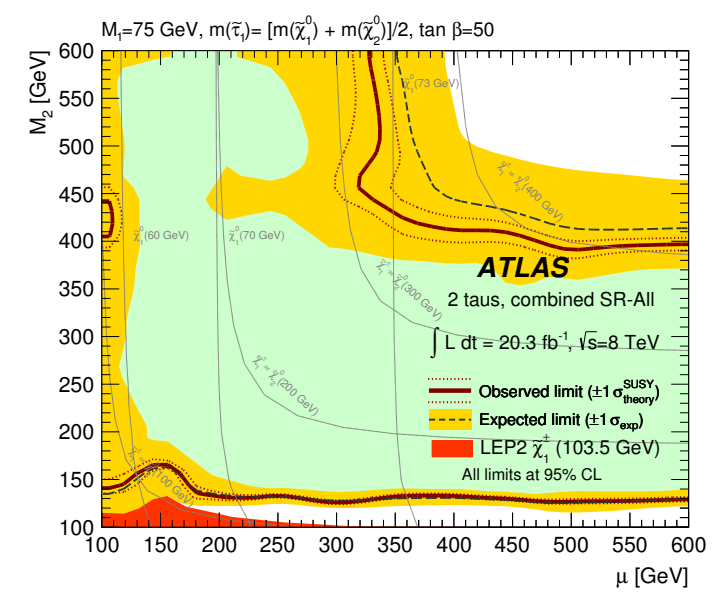

(b) pMSSM model (variable stau mass)

Figure 10. 95\% CL exclusion limits in the $\mu-M_{2}$ mass plane for the pMSSM models with (a) fixed and (b) variable stau mass. See text for details of exclusion curves and uncertainty bands. The areas excluded by the $-1 \sigma$ expected limit are shown in green. The LEP limit $[39,40]$ on the mass of the chargino is also shown in red.

be expected to be similar also at large $\mu\left(M_{2}\right)$ for values of $M_{2}(\mu)$ in the range 150-350 (100-300) GeV.

In the pMSSM model with fixed stau mass, SR-DS-highMass provides better exclusion at high $\mu, M_{2}$. For $M_{2}, \mu<200 \mathrm{GeV}$, SR-C1N2 and SR-C1C1 provide the most stringent limits. In the pMSSM model with variable stau mass, SR-C1N2 and SR-DS-highMass give the best sensitivity in the whole parameter space.

\section{Conclusion}

Searches for the electroweak production of supersymmetric particles in events with at least two hadronically decaying taus, missing transverse momentum and low jet activity in the final state are performed using $20.3 \mathrm{fb}^{-1}$ of proton-proton collision data at $\sqrt{s}=8 \mathrm{TeV}$ recorded with the ATLAS experiment at the Large Hadron Collider. Agreement between data and SM expectations is observed in all signal regions. These results are used to set limits on the visible cross section for non-SM events in each signal region. Exclusion limits are placed on parameters of the pMSSM and simplified models.

For simplified models, chargino masses up to $345 \mathrm{GeV}$ are excluded for a massless lightest neutralino in the scenario of direct production of wino-like chargino pairs, with each chargino decaying into the lightest neutralino via an intermediate on-shell stau. In the case of associated production of mass-degenerate charginos and next-to-lightest neutralinos, masses up to $410 \mathrm{GeV}$ are excluded for a massless lightest neutralino. In pMSSM models the excluded $\tilde{\chi}_{1}^{ \pm}\left(\tilde{\chi}_{2}^{0}\right)$ mass range is between 100 and $350 \mathrm{GeV}$. For direct stau production, the best upper limit on the signal strength is found for a mass of the $\tilde{\tau}_{\mathrm{R}}\left(\tilde{\tau}_{\mathrm{L}}\right)$ of $90.6(93.1) \mathrm{GeV}$ and a massless $\tilde{\chi}_{1}^{0}$. The excluded cross section for $\tilde{\tau}_{\mathrm{R}} \tilde{\tau}_{\mathrm{R}}\left(\tilde{\tau}_{\mathrm{L}} \tilde{\tau}_{\mathrm{L}}\right)$ is $0.22(0.28)$ pb for this combination of stau and lightest supersymmetric particle masses, while the theoretical cross section at NLO is $0.07(0.17) \mathrm{pb}$. 


\section{Acknowledgments}

We thank CERN for the very successful operation of the LHC, as well as the support staff from our institutions without whom ATLAS could not be operated efficiently.

We acknowledge the support of ANPCyT, Argentina; YerPhI, Armenia; ARC, Australia; BMWF and FWF, Austria; ANAS, Azerbaijan; SSTC, Belarus; CNPq and FAPESP, Brazil; NSERC, NRC and CFI, Canada; CERN; CONICYT, Chile; CAS, MOST and NSFC, China; COLCIENCIAS, Colombia; MSMT CR, MPO CR and VSC CR, Czech Republic; DNRF, DNSRC and Lundbeck Foundation, Denmark; EPLANET, ERC and NSRF, European Union; IN2P3-CNRS, CEA-DSM/IRFU, France; GNSF, Georgia; BMBF, DFG, HGF, MPG and AvH Foundation, Germany; GSRT and NSRF, Greece; ISF, MINERVA, GIF, I-CORE and Benoziyo Center, Israel; INFN, Italy; MEXT and JSPS, Japan; CNRST, Morocco; FOM and NWO, Netherlands; BRF and RCN, Norway; MNiSW and NCN, Poland; GRICES and FCT, Portugal; MNE/IFA, Romania; MES of Russia and ROSATOM, Russian Federation; JINR; MSTD, Serbia; MSSR, Slovakia; ARRS and MIZŠ, Slovenia; DST/NRF, South Africa; MINECO, Spain; SRC and Wallenberg Foundation, Sweden; SER, SNSF and Cantons of Bern and Geneva, Switzerland; NSC, Taiwan; TAEK, Turkey; STFC, the Royal Society and Leverhulme Trust, United Kingdom; DOE and NSF, United States of America.

The crucial computing support from all WLCG partners is acknowledged gratefully, in particular from CERN and the ATLAS Tier-1 facilities at TRIUMF (Canada), NDGF (Denmark, Norway, Sweden), CC-IN2P3 (France), KIT/GridKA (Germany), INFN-CNAF (Italy), NL-T1 (Netherlands), PIC (Spain), ASGC (Taiwan), RAL (U.K.) and BNL (U.S.A.) and in the Tier-2 facilities worldwide.

Open Access. This article is distributed under the terms of the Creative Commons Attribution License (CC-BY 4.0), which permits any use, distribution and reproduction in any medium, provided the original author(s) and source are credited.

\section{References}

[1] H. Miyazawa, Baryon number changing currents, Prog. Theor. Phys. 36 (1966) 1266.

[2] P. Ramond, Dual theory for free fermions, Phys. Rev. D 3 (1971) 2415 [InSPIRE].

[3] Y. Golfand and E.P. Likhtman, Extension of the algebra of Poincaré group generators and violation of p invariance, JETP Lett. 13 (1971) 323 [Pisma Zh. Eksp. Teor. Fiz. 13 (1971) 452] [INSPIRE].

[4] A. Neveu and J.H. Schwarz, Factorizable dual model of pions, Nucl. Phys. B 31 (1971) 86 [INSPIRE].

[5] A. Neveu and J.H. Schwarz, Quark model of dual pions, Phys. Rev. D 4 (1971) 1109 [INSPIRE].

[6] J.-L. Gervais and B. Sakita, Field theory interpretation of supergauges in dual models, Nucl. Phys. B 34 (1971) 632 [INSPIRE]. 
[7] D.V. Volkov and V.P. Akulov, Is the neutrino a Goldstone particle?, Phys. Lett. B 46 (1973) 109 [INSPIRE].

[8] J. Wess and B. Zumino, A lagrangian model invariant under supergauge transformations, Phys. Lett. B 49 (1974) 52 [inSPIRE].

[9] J. Wess and B. Zumino, Supergauge transformations in four-dimensions, Nucl. Phys. B 70 (1974) 39 [INSPIRE].

[10] S. Weinberg, Implications of dynamical symmetry breaking, Phys. Rev. D 13 (1976) 974 [INSPIRE].

[11] E. Gildener, Gauge symmetry hierarchies, Phys. Rev. D 14 (1976) 1667 [InSPIRE].

[12] S. Weinberg, Implications of dynamical symmetry breaking: an addendum, Phys. Rev. D 19 (1979) 1277 [INSPIRE].

[13] L. Susskind, Dynamics of spontaneous symmetry breaking in the Weinberg-Salam theory, Phys. Rev. D 20 (1979) 2619 [inSPIRE].

[14] P. Fayet, Supersymmetry and weak, electromagnetic and strong interactions, Phys. Lett. B 64 (1976) 159 [INSPIRE].

[15] P. Fayet, Spontaneously broken supersymmetric theories of weak, electromagnetic and strong interactions, Phys. Lett. B 69 (1977) 489 [INSPIRE].

[16] G.R. Farrar and P. Fayet, Phenomenology of the production, decay and detection of new hadronic states associated with supersymmetry, Phys. Lett. B 76 (1978) 575 [InSPIRE].

[17] P. Fayet, Relations between the masses of the superpartners of leptons and quarks, the Goldstino couplings and the neutral currents, Phys. Lett. B 84 (1979) 416 [INSPIRE].

[18] S. Dimopoulos and H. Georgi, Softly broken supersymmetry and SU(5), Nucl. Phys. B 193 (1981) 150 [INSPIRE].

[19] G. Jungman, M. Kamionkowski and K. Griest, Supersymmetric dark matter, Phys. Rep. 267 (1996) 195 [hep-ph/9506380].

[20] H. Goldberg, Constraint on the photino mass from cosmology, Phys. Rev. Lett. 50 (1983) 1419 [Erratum ibid. 103 (2009) 099905] [INSPIRE].

[21] J.R. Ellis, J.S. Hagelin, D.V. Nanopoulos, K.A. Olive and M. Srednicki, Supersymmetric relics from the Big Bang, Nucl. Phys. B 238 (1984) 453 [InSPIRE].

[22] L. Evans and P. Bryant, LHC machine, 2008 JINST 3 S08001 [INSPIRE].

[23] R. Barbieri and G.F. Giudice, Upper bounds on supersymmetric particle masses, Nucl. Phys. B 306 (1988) 63 [INSPIRE].

[24] B. de Carlos and J.A. Casas, One loop analysis of the electroweak breaking in supersymmetric models and the fine tuning problem, Phys. Lett. B 309 (1993) 320 [hep-ph/9303291] [INSPIRE].

[25] G. Bélanger, F. Boudjema, A. Cottrant, A. Pukhov and A. Semenov, WMAP constraints on SUGRA models with non-universal gaugino masses and prospects for direct detection, Nucl. Phys. B 706 (2005) 411 [hep-ph/0407218] [INSPIRE].

[26] S.F. King, J.P. Roberts and D.P. Roy, Natural dark matter in SUSY GUTs with non-universal gaugino masses, JHEP 10 (2007) 106 [arXiv:0705.4219] [INSPIRE]. 
[27] M. Dine and W. Fischler, A phenomenological model of particle physics based on supersymmetry, Phys. Lett. B 110 (1982) 227 [INSPIRE].

[28] L. Álvarez-Gaumé, M. Claudson and M.B. Wise, Low-energy supersymmetry, Nucl. Phys. B 207 (1982) 96 [InSPIRE].

[29] C.R. Nappi and B.A. Ovrut, Supersymmetric extension of the $\mathrm{SU}(3) \times \mathrm{SU}(2) \times \mathrm{U}(1)$ model, Phys. Lett. B 113 (1982) 175 [inSPIRE].

[30] M. Dine and A.E. Nelson, Dynamical supersymmetry breaking at low-energies, Phys. Rev. D 48 (1993) 1277 [hep-ph/9303230] [INSPIRE].

[31] M. Dine, A.E. Nelson and Y. Shirman, Low-energy dynamical supersymmetry breaking simplified, Phys. Rev. D 51 (1995) 1362 [hep-ph/9408384] [INSPIRE].

[32] M. Dine, A.E. Nelson, Y. Nir and Y. Shirman, New tools for low-energy dynamical supersymmetry breaking, Phys. Rev. D 53 (1996) 2658 [hep-ph/9507378] [INSPIRE].

[33] L. Randall and R. Sundrum, Out of this world supersymmetry breaking, Nucl. Phys. B 557 (1999) 79 [hep-th/9810155] [INSPIRE].

[34] G.F. Giudice, M.A. Luty, H. Murayama and R. Rattazzi, Gaugino mass without singlets, JHEP 12 (1998) 027 [hep-ph/9810442] [INSPIRE].

[35] D. Albornoz Vasquez, G. Bélanger and C. Boehm, Revisiting light neutralino scenarios in the MSSM, Phys. Rev. D 84 (2011) 095015 [arXiv:1108.1338] [INSPIRE].

[36] ATLAS collaboration, Search for direct production of charginos, neutralinos and sleptons in final states with two leptons and missing transverse momentum in pp collisions at $\sqrt{s}=8 \mathrm{TeV}$ with the ATLAS detector, JHEP 05 (2014) 071 [arXiv: 1403.5294] [INSPIRE].

[37] ATLAS collaboration, Search for direct production of charginos and neutralinos in events with three leptons and missing transverse momentum in $\sqrt{s}=8 \mathrm{TeV}$ pp collisions with the ATLAS detector, JHEP 04 (2014) 169 [arXiv: 1402.7029] [INSPIRE].

[38] CMS collaboration, Searches for electroweak production of charginos, neutralinos and sleptons decaying to leptons and $W, Z$ and Higgs bosons in pp collisions at $8 \mathrm{TeV}$, Eur. Phys. J. C 74 (2014) 3036 [arXiv: 1405.7570] [INSPIRE].

[39] LEP SUSY working group, Combined LEP chargino results, up to $208 \mathrm{GeV}$ for large $m_{0}$, LEPSUSYWG/01-03.1 (2001).

[40] LEP SUSY working group, Combined LEP selectron/smuon/stau results, 183-208 GeV, LEPSUSYWG/04-01.1 (2004).

[41] DELPHI collaboration, J. Abdallah et al., Searches for supersymmetric particles in $e^{+} e^{-}$ collisions up to $208 \mathrm{GeV}$ and interpretation of the results within the MSSM, Eur. Phys. J. C 31 (2003) 421 [hep-ex/0311019] [INSPIRE].

[42] L3 collaboration, M. Acciarri et al., Search for charginos and neutralinos in $e^{+} e^{-}$collisions at $\sqrt{s}=189 \mathrm{GeV}$, Phys. Lett. B 472 (2000) 420 [hep-ex/9910007] [INSPIRE].

[43] OPAL collaboration, G. Abbiendi et al., Search for chargino and neutralino production at $\sqrt{s}=192 \mathrm{GeV}$ to $209 \mathrm{GeV}$ at LEP, Eur. Phys. J. C 35 (2004) 1 [hep-ex/0401026] [INSPIRE].

[44] A. Djouadi, J.-L. Kneur and G. Moultaka, SuSpect: A Fortran code for the supersymmetric and Higgs particle spectrum in the MSSM, Comput. Phys. Commun. 176 (2007) 426 [hep-ph/0211331] [INSPIRE]. 
[45] C.F. Berger, J.S. Gainer, J.L. Hewett and T.G. Rizzo, Supersymmetry without prejudice, JHEP 02 (2009) 023 [arXiv:0812.0980] [InSPIRE].

[46] S.S. AbdusSalam, B.C. Allanach, F. Quevedo, F. Feroz and M. Hobson, Fitting the phenomenological MSSM, Phys. Rev. D 81 (2010) 095012 [arXiv:0904.2548] [INSPIRE].

[47] J. Alwall, P. Schuster and N. Toro, Simplified models for a first characterization of new physics at the LHC, Phys. Rev. D 79 (2009) 075020 [arXiv:0810.3921] [INSPIRE].

[48] LhC New Physics Working Group collaboration, D. Alves et al., Simplified models for LHC new physics searches, J. Phys. G 39 (2012) 105005 [arXiv:1105.2838] [inSPIRE].

[49] H. Baer, C.-h. Chen, F. Paige and X. Tata, Detecting sleptons at hadron colliders and supercolliders, Phys. Rev. D 49 (1994) 3283 [hep-ph/9311248] [INSPIRE].

[50] ATLAS collaboration, The ATLAS experiment at the CERN Large Hadron Collider, 2008 JINST 3 S08003 [INSPIRE].

[51] ATLAS collaboration, Improved luminosity determination in pp collisions at $\sqrt{s}=7 \mathrm{TeV}$ using the ATLAS detector at the LHC, Eur. Phys. J. C 73 (2013) 2518 [arXiv:1302.4393] [INSPIRE].

[52] ATLAS collaboration, Performance of the ATLAS tau trigger in 2011, ATLAS-CONF-2013-006 (2013).

[53] ATLAS collaboration, The ATLAS simulation infrastructure, Eur. Phys. J. C 70 (2010) 823 [arXiv: 1005.4568] [INSPIRE].

[54] GEANT4 collaboration, S. Agostinelli et al., GEANT4: a simulation toolkit, Nucl. Instrum. Meth. A 506 (2003) 250 [inSPIRE].

[55] T. Gleisberg et al., Event generation with SHERPA 1.1, JHEP 02 (2009) 007 [arXiv:0811.4622] [INSPIRE].

[56] T. Binoth, M. Ciccolini, N. Kauer and M. Krämer, Gluon-induced W-boson pair production at the LHC, JHEP 12 (2006) 046 [hep-ph/0611170] [INSPIRE].

[57] J. Alwall, M. Herquet, F. Maltoni, O. Mattelaer and T. Stelzer, MadGraph 5: going beyond, JHEP 06 (2011) 128 [arXiv:1106.0522] [INSPIRE].

[58] T. Sjöstrand, S. Mrenna and P.Z. Skands, PYTHIA 6.4 physics and manual, JHEP 05 (2006) 026 [hep-ph/0603175] [INSPIRE].

[59] T. Sjöstrand, S. Mrenna and P.Z. Skands, A brief introduction to PYTHIA 8.1, Comput. Phys. Commun. 178 (2008) 852 [arXiv:0710. 3820] [INSPIRE].

[60] S. Frixione and B.R. Webber, Matching NLO QCD computations and parton shower simulations, JHEP 06 (2002) 029 [hep-ph/0204244] [INSPIRE].

[61] S. Frixione, P. Nason and B.R. Webber, Matching NLO QCD and parton showers in heavy flavor production, JHEP 08 (2003) 007 [hep-ph/0305252] [INSPIRE].

[62] S. Frixione, E. Laenen, P. Motylinski and B.R. Webber, Single-top production in MC@NLO, JHEP 03 (2006) 092 [hep-ph/0512250] [INSPIRE].

[63] B.P. Kersevan and E. Richter-Was, The Monte Carlo event generator AcerMC version 1.0 with interfaces to PYTHIA 6.2 and HERWIG 6.3, Comput. Phys. Commun. 149 (2003) 142 [hep-ph/0201302] [INSPIRE]. 
[64] M.L. Mangano, M. Moretti, F. Piccinini, R. Pittau and A.D. Polosa, ALPGEN, a generator for hard multiparton processes in hadronic collisions, JHEP 07 (2003) 001 [hep-ph/0206293] [INSPIRE].

[65] S. Frixione, P. Nason and C. Oleari, Matching NLO QCD computations with Parton Shower simulations: the POWHEG method, JHEP 11 (2007) 070 [arXiv: 0709.2092] [INSPIRE].

[66] ATLAS collaboration, ATLAS tunes of PYTHIA 6 and Pythia 8 for MC11, ATLAS-PHYS-PUB-2011-009 (2011).

[67] P.Z. Skands, Tuning Monte Carlo generators: the Perugia tunes, Phys. Rev. D 82 (2010) 074018 [arXiv: 1005.3457] [INSPIRE].

[68] H.-L. Lai et al., New parton distributions for collider physics, Phys. Rev. D 82 (2010) 074024 [arXiv: 1007.2241] [INSPIRE].

[69] J. Pumplin et al., New generation of parton distributions with uncertainties from global QCD analysis, JHEP 07 (2002) 012 [hep-ph/0201195] [INSPIRE].

[70] S. Catani, L. Cieri, G. Ferrera, D. de Florian and M. Grazzini, Vector boson production at hadron colliders: a fully exclusive QCD calculation at NNLO, Phys. Rev. Lett. 103 (2009) 082001 [arXiv: 0903.2120] [INSPIRE].

[71] A.D. Martin, W.J. Stirling, R.S. Thorne and G. Watt, Update of parton distributions at NNLO, Phys. Lett. B 652 (2007) 292 [arXiv:0706.0459] [INSPIRE].

[72] M. Czakon and A. Mitov, Top++: a program for the calculation of the top-pair cross-section at hadron colliders, Comput. Phys. Commun. 185 (2014) 2930 [arXiv:1112.5675] [INSPIRE].

[73] J.M. Campbell and R.K. Ellis, An update on vector boson pair production at hadron colliders, Phys. Rev. D 60 (1999) 113006 [hep-ph/9905386] [INSPIRE].

[74] J.M. Campbell, R.K. Ellis and C. Williams, Vector boson pair production at the LHC, JHEP 07 (2011) 018 [arXiv:1105.0020] [InSPIRE].

[75] J.M. Campbell and R.K. Ellis, $t \bar{t} W^{ \pm}$production and decay at NLO, JHEP 07 (2012) 052 [arXiv: 1204.5678] [INSPIRE].

[76] M.V. Garzelli, A. Kardos, C.G. Papadopoulos and Z. Trócsányi, $t \bar{t} W^{ \pm}$and $t \bar{t} Z$ hadroproduction at NLO accuracy in QCD with parton shower and hadronization effects, JHEP 11 (2012) 056 [arXiv:1208.2665] [INSPIRE].

[77] M. Bahr et al., HERWIG++ physics and manual, Eur. Phys. J. C 58 (2008) 639 [arXiv:0803.0883] [INSPIRE].

[78] W. Beenakker et al., The production of charginos/neutralinos and sleptons at hadron colliders, Phys. Rev. Lett. 83 (1999) 3780 [Erratum ibid. 100 (2008) 029901] [hep-ph/9906298] [INSPIRE].

[79] B. Fuks, M. Klasen, D.R. Lamprea and M. Rothering, Gaugino production in proton-proton collisions at a center-of-mass energy of 8 TeV, JHEP 10 (2012) 081 [arXiv:1207.2159] [INSPIRE].

[80] B. Fuks, M. Klasen, D.R. Lamprea and M. Rothering, Precision predictions for electroweak superpartner production at hadron colliders with Resummino, Eur. Phys. J. C 73 (2013) 2480 [arXiv: 1304.0790] [INSPIRE]. 
[81] B. Fuks, M. Klasen, D.R. Lamprea and M. Rothering, Revisiting slepton pair production at the Large Hadron Collider, JHEP 01 (2014) 168 [arXiv: 1310.2621] [INSPIRE].

[82] S.S. AbdusSalam et al., Benchmark models, planes, lines and points for future SUSY searches at the LHC, Eur. Phys. J. C 71 (2011) 1835 [arXiv:1109.3859] [InSPIRE].

[83] M. Cacciari, G.P. Salam and G. Soyez, The anti- $k_{t}$ jet clustering algorithm, JHEP 04 (2008) 063 [arXiv:0802.1189] [INSPIRE].

[84] M. Cacciari and G.P. Salam, Dispelling the $N^{3}$ myth for the $k_{t}$ jet-finder, Phys. Lett. B 641 (2006) 57 [hep-ph/0512210] [INSPIRE].

[85] ATLAS collaboration, Jet energy measurement with the ATLAS detector in proton-proton collisions at $\sqrt{s}=7 \mathrm{TeV}$, Eur. Phys. J. C 73 (2013) 2304 [arXiv:1112.6426] [InSPIRE].

[86] M. Cacciari and G.P. Salam, Pileup subtraction using jet areas, Phys. Lett. B 659 (2008) 119 [arXiv:0707.1378] [inSPIRE].

[87] ATLAS collaboration, Electron performance measurements with the ATLAS detector using the 2010 LHC proton-proton collision data, Eur. Phys. J. C 72 (2012) 1909 [arXiv: 1110.3174] [INSPIRE].

[88] ATLAS collaboration, Preliminary results on the muon reconstruction efficiency, momentum resolution and momentum scale in ATLAS 2012 pp collision data, ATLAS-CONF-2013-088 (2013).

[89] ATLAS collaboration, Determination of the $\tau$ energy scale and the associated systematic uncertainty in proton-proton collisions at $\sqrt{s}=8 \mathrm{TeV}$ with the ATLAS detector at the LHC in 2012, ATLAS-CONF-2013-044 (2013).

[90] ATLAS collaboration, Identification of the hadronic decays of $\tau$ leptons in 2012 data with the ATLAS detector, ATLAS-CONF-2013-064 (2013).

[91] ATLAS collaboration, Performance of missing transverse momentum reconstruction in proton-proton collisions at $7 \mathrm{TeV}$ with ATLAS, Eur. Phys. J. C 72 (2012) 1844 [arXiv: 1108.5602] [INSPIRE].

[92] ATLAS collaboration, Commissioning of the ATLAS high-performance b-tagging algorithms in the $7 \mathrm{TeV}$ collision data, ATLAS-CONF-2011-102 (2011).

[93] ATLAS collaboration, Electron reconstruction and identification efficiency measurements with the ATLAS detector using the 2011 LHC proton-proton collision data, Eur. Phys. J. C 74 (2014) 2941 [arXiv:1404.2240] [INSPIRE].

[94] C.G. Lester and D.J. Summers, Measuring masses of semiinvisibly decaying particles pair produced at hadron colliders, Phys. Lett. B 463 (1999) 99 [hep-ph/9906349] [INSPIRE].

[95] A. Barr, C. Lester and P. Stephens, $m_{T 2}$ : the truth behind the glamour, J. Phys. G 29 (2003) 2343 [hep-ph/0304226] [INSPIRE].

[96] G. Cowan, K. Cranmer, E. Gross and O. Vitells, Asymptotic formulae for likelihood-based tests of new physics, Eur. Phys. J. C 71 (2011) 1554 [arXiv:1007.1727] [InSPIRE].

[97] D.R. Tovey, On measuring the masses of pair-produced semi-invisibly decaying particles at hadron colliders, JHEP 04 (2008) 034 [arXiv:0802.2879] [INSPIRE].

[98] G. Polesello and D.R. Tovey, Supersymmetric particle mass measurement with the boost-corrected contransverse mass, JHEP 03 (2010) 030 [arXiv:0910.0174] [INSPIRE]. 
[99] ATLAS collaboration, Measuring the b-tag efficiency in a top-pair sample with $4.7 \mathrm{fb}^{-1}$ of data from the ATLAS detector, ATLAS-CONF-2012-097 (2012).

[100] J. Alwall et al., Comparative study of various algorithms for the merging of parton showers and matrix elements in hadronic collisions, Eur. Phys. J. C 53 (2008) 473 [arXiv:0706.2569] [INSPIRE].

[101] M. Krämer et al., Supersymmetry production cross sections in pp collisions at $\sqrt{s}=7 \mathrm{TeV}$, arXiv: 1206.2892 [INSPIRE].

[102] A.L. Read, Presentation of search results: the CL(s) technique, J. Phys. G 28 (2002) 2693 [INSPIRE]. 


\section{The ATLAS collaboration}

G. Aad $^{84}$, B. Abbott ${ }^{112}$, J. Abdallah ${ }^{152}$, S. Abdel Khalek ${ }^{116}$, O. Abdinov ${ }^{11}$, R. Aben ${ }^{106}$, B. Abi ${ }^{113}$, M. Abolins ${ }^{89}$, O.S. AbouZeid ${ }^{159}$, H. Abramowicz ${ }^{154}$, H. Abreu ${ }^{153}$, R. Abreu ${ }^{30}$, Y. Abulaiti 147a,147b, B.S. Acharya ${ }^{165 a, 165 b, a}$, L. Adamczyk ${ }^{38 a}$, D.L. Adams ${ }^{25}$, J. Adelman ${ }^{177}$, S. Adomeit ${ }^{99}$, T. Adye ${ }^{130}$, T. Agatonovic-Jovin ${ }^{13 a}$, J.A. Aguilar-Saavedra ${ }^{125 a, 125 f}$, M. Agustoni ${ }^{17}$, S.P. Ahlen ${ }^{22}$, F. Ahmadov ${ }^{64, b}$, G. Aielli ${ }^{134 a, 134 b}$, H. Akerstedt ${ }^{147 a, 147 b}$, T.P.A. Åkesson ${ }^{80}$, G. Akimoto ${ }^{156}$, A.V. Akimov ${ }^{95}$, G.L. Alberghi ${ }^{20 a, 20 b}$, J. Albert ${ }^{170}$, S. Albrand ${ }^{55}$, M.J. Alconada Verzini ${ }^{70}$, M. Aleksa ${ }^{30}$, I.N. Aleksandrov ${ }^{64}$, C. Alexa ${ }^{26 a}$, G. Alexander ${ }^{154}$, G. Alexandre ${ }^{49}$, T. Alexopoulos ${ }^{10}$, M. Alhroob ${ }^{165 a, 165 c}$, G. Alimonti ${ }^{90 a}$, L. Alio ${ }^{84}$, J. Alison ${ }^{31}$, B.M.M. Allbrooke ${ }^{18}$, L.J. Allison ${ }^{71}$, P.P. Allport ${ }^{73}$, J. Almond ${ }^{83}$, A. Aloisio 103a,103b , A. Alonso ${ }^{36}$, F. Alonso ${ }^{70}$, C. Alpigiani ${ }^{75}$, A. Altheimer ${ }^{35}$, B. Alvarez Gonzalez ${ }^{89}$, M.G. Alviggi103a,103b,

K. Amako ${ }^{65}$, Y. Amaral Coutinho ${ }^{24 a}$, C. Amelung ${ }^{23}$, D. Amidei ${ }^{88}$, S.P. Amor Dos Santos ${ }^{125 a, 125 c}$, A. Amorim $125 \mathrm{a}, 125 \mathrm{~b}, \mathrm{~S}$. Amoroso ${ }^{48}$, N. Amram ${ }^{154}$, G. Amundsen ${ }^{23}$, C. Anastopoulos ${ }^{140}$, L.S. Ancu ${ }^{49}$, N. Andari ${ }^{30}$, T. Andeen ${ }^{35}$, C.F. Anders ${ }^{58 b}$, G. Anders ${ }^{30}$, K.J. Anderson ${ }^{31}$, A. Andreazza ${ }^{90 a, 90 b}$, V. Andrei ${ }^{58 a}$, X.S. Anduaga ${ }^{70}$, S. Angelidakis ${ }^{9}$, I. Angelozzi ${ }^{106}$, P. Anger ${ }^{44}$, A. Angerami ${ }^{35}$, F. Anghinolfi ${ }^{30}$, A.V. Anisenkov ${ }^{108}$, N. Anjos ${ }^{125 a}$, A. Annovi ${ }^{47}$, A. Antonaki ${ }^{9}$, M. Antonelli ${ }^{47}$, A. Antonov ${ }^{97}$, J. Antos ${ }^{145 b}$, F. Anulli ${ }^{133 a}$, M. Aoki ${ }^{65}$, L. Aperio Bella ${ }^{18}$, R. Apolle ${ }^{119, c}$, G. Arabidze ${ }^{89}$, I. Aracena ${ }^{144}$, Y. Arai ${ }^{65}$, J.P. Araque ${ }^{125 a}$, A.T.H. Arce ${ }^{45}$, J-F. Arguin ${ }^{94}$, S. Argyropoulos ${ }^{42}$, M. Arik ${ }^{19 a}$, A.J. Armbruster ${ }^{30}$, O. Arnaez ${ }^{30}$, V. Arnal ${ }^{81}$,

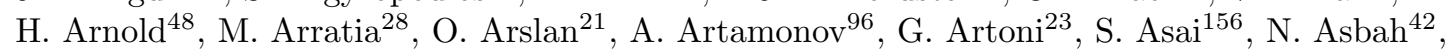
A. Ashkenazi ${ }^{154}$, B. Åsman 147a,147b , L. Asquith ${ }^{6}$, K. Assamagan ${ }^{25}$, R. Astalos ${ }^{145 a}$,

M. Atkinson ${ }^{166}$, N.B. Atlay ${ }^{142}$, B. Auerbach ${ }^{6}$, K. Augsten ${ }^{127}$, M. Aurousseau ${ }^{146 b}$, G. Avolio ${ }^{30}$, G. Azuelos ${ }^{94, d}$, Y. Azuma ${ }^{156}$, M.A. Baak ${ }^{30}$, A. Baas ${ }^{58 a}$, C. Bacci ${ }^{135 a, 135 b}$, H. Bachacou ${ }^{137}$,

K. Bachas ${ }^{155}$, M. Backes ${ }^{30}$, M. Backhaus ${ }^{30}$, J. Backus Mayes ${ }^{144}$, E. Badescu ${ }^{26 a}$,

P. Bagiacchi133a,133b, P. Bagnaia ${ }^{133 a, 133 b}$, Y. Bai ${ }^{33 a}$, T. Bain ${ }^{35}$, J.T. Baines ${ }^{130}$, O.K. Baker ${ }^{177}$, P. Balek ${ }^{128}$, F. Balli ${ }^{137}$, E. Banas ${ }^{39}$, Sw. Banerjee ${ }^{174}$, A.A.E. Bannoura ${ }^{176}$, V. Bansal ${ }^{170}$, H.S. Bansil ${ }^{18}$, L. Barak ${ }^{173}$, S.P. Baranov ${ }^{95}$, E.L. Barberio ${ }^{87}$, D. Barberis ${ }^{50 a, 50 b}$, M. Barbero ${ }^{84}$, T. Barillari ${ }^{100}$, M. Barisonzi ${ }^{176}$, T. Barklow ${ }^{144}$, N. Barlow ${ }^{28}$, B.M. Barnett ${ }^{130}$, R.M. Barnett ${ }^{15}$, Z. Barnovska ${ }^{5}$, A. Baroncelli ${ }^{135 a}$, G. Barone ${ }^{49}$, A.J. Barr ${ }^{119}$, F. Barreiro ${ }^{81}$,

J. Barreiro Guimarães da Costa ${ }^{57}$, R. Bartoldus ${ }^{144}$, A.E. Barton ${ }^{71}$, P. Bartos ${ }^{145 a}$, V. Bartsch ${ }^{150}$,

A. Bassalat ${ }^{116}$, A. Basye ${ }^{166}$, R.L. Bates ${ }^{53}$, J.R. Batley ${ }^{28}$, M. Battaglia ${ }^{138}$, M. Battistin ${ }^{30}$,

F. Bauer ${ }^{137}$, H.S. Bawa ${ }^{144, e}$, M.D. Beattie ${ }^{71}$, T. Beau ${ }^{79}$, P.H. Beauchemin ${ }^{162}$,

R. Beccherle ${ }^{123 a, 123 b}$, P. Bechtle ${ }^{21}$, H.P. Beck ${ }^{17}$, K. Becker ${ }^{176}$, S. Becker ${ }^{99}$, M. Beckingham ${ }^{171}$, C. Becot $^{116}$, A.J. Beddall ${ }^{19 c}$, A. Beddall ${ }^{19 c}$, S. Bedikian ${ }^{177}$, V.A. Bednyakov ${ }^{64}$, C.P. Bee ${ }^{149}$, L.J. Beemster ${ }^{106}$, T.A. Beermann ${ }^{176}$, M. Begel ${ }^{25}$, K. Behr ${ }^{119}$, C. Belanger-Champagne ${ }^{86}$, P.J. Bell ${ }^{49}$, W.H. Bell ${ }^{49}$, G. Bella ${ }^{154}$, L. Bellagamba ${ }^{20 a}$, A. Bellerive ${ }^{29}$, M. Bellomo ${ }^{85}$, K. Belotskiy ${ }^{97}$, O. Beltramello ${ }^{30}$, O. Benary ${ }^{154}$, D. Benchekroun ${ }^{136 a}$, K. Bendtz ${ }^{147 a, 147 b}$, N. Benekos ${ }^{166}$, Y. Benhammou ${ }^{154}$, E. Benhar Noccioli ${ }^{49}$, J.A. Benitez Garcia ${ }^{160 b}$, D.P. Benjamin ${ }^{45}$, J.R. Bensinger ${ }^{23}$, K. Benslama ${ }^{131}$, S. Bentvelsen ${ }^{106}$, D. Berge ${ }^{106}$, E. Bergeaas Kuutmann ${ }^{16}$, N. Berger ${ }^{5}$, F. Berghaus ${ }^{170}$, J. Beringer ${ }^{15}$, C. Bernard ${ }^{22}$, P. Bernat ${ }^{77}$, C. Bernius ${ }^{78}$, F.U. Bernlochner ${ }^{170}$, T. Berry ${ }^{76}$, P. Berta ${ }^{128}$, C. Bertella ${ }^{84}$, G. Bertoli ${ }^{147 a, 147 b}$, F. Bertolucci ${ }^{123 a, 123 b}$, C. Bertsche ${ }^{112}$, D. Bertsche ${ }^{112}$, M.I. Besana ${ }^{90 a}$, G.J. Besjes ${ }^{105}$, O. Bessidskaia ${ }^{147 a, 147 b}$, M.F. Bessner ${ }^{42}$, N. Besson ${ }^{137}$, C. Betancourt ${ }^{48}$, S. Bethke ${ }^{100}$, W. Bhimji ${ }^{46}$, R.M. Bianchi ${ }^{124}$, L. Bianchini ${ }^{23}$, M. Bianco ${ }^{30}$, O. Biebel ${ }^{99}$, S.P. Bieniek ${ }^{77}$, K. Bierwagen ${ }^{54}$, J. Biesiada ${ }^{15}$, M. Biglietti ${ }^{135 a}$, J. Bilbao De Mendizabal ${ }^{49}$, H. Bilokon ${ }^{47}$, M. Bindi ${ }^{54}$, S. Binet ${ }^{116}$, A. Bingul ${ }^{19 c}$, C. Bini ${ }^{133 a, 133 b}$, C.W. Black ${ }^{151}$, J.E. Black ${ }^{144}$, K.M. Black ${ }^{22}$, D. Blackburn ${ }^{139}$, R.E. Blair 6 , J.-B. Blanchard ${ }^{137}$, T. Blazek ${ }^{145 a}$, I. Bloch ${ }^{42}$, C. Blocker ${ }^{23}$, W. Blum ${ }^{82, *}$, U. Blumenschein ${ }^{54}$, G.J. Bobbink ${ }^{106}$, V.S. Bobrovnikov ${ }^{108}$, S.S. Bocchetta ${ }^{80}$, A. Bocci ${ }^{45}$, C. Bock ${ }^{99}$, C.R. Boddy ${ }^{119}$, M. Boehler ${ }^{48}$, T.T. Boek ${ }^{176}$, J.A. Bogaerts ${ }^{30}$, A.G. Bogdanchikov ${ }^{108}$, A. Bogouch ${ }^{91, *}$, C. Bohm ${ }^{147 a}$, J. Bohm ${ }^{126}$, V. Boisvert ${ }^{76}$, T. Bold ${ }^{38 a}$, V. Boldea ${ }^{26 a}$, A.S. Boldyrev ${ }^{98}$, M. Bomben ${ }^{79}$, M. Bona ${ }^{75}$, M. Boonekamp ${ }^{137}$,

A. Borisov ${ }^{129}$, G. Borissov ${ }^{71}$, M. Borri ${ }^{83}$, S. Borroni ${ }^{42}$, J. Bortfeldt ${ }^{99}$, V. Bortolotto ${ }^{135 a, 135 b}$, 
K. Bos ${ }^{106}$, D. Boscherini ${ }^{20 a}$, M. Bosman ${ }^{12}$, H. Boterenbrood ${ }^{106}$, J. Boudreau ${ }^{124}$, J. Bouffard ${ }^{2}$, E.V. Bouhova-Thacker ${ }^{71}$, D. Boumediene ${ }^{34}$, C. Bourdarios ${ }^{116}$, N. Bousson ${ }^{113}$, S. Boutouil ${ }^{136 d}$,

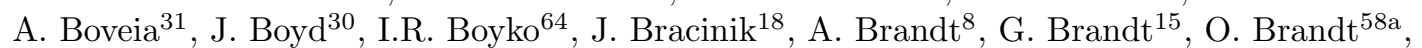
U. Bratzler ${ }^{157}$, B. Brau ${ }^{85}$, J.E. Brau ${ }^{115}$, H.M. Braun ${ }^{176, *}$, S.F. Brazzale ${ }^{165 a, 165 c}$, B. Brelier ${ }^{159}$, K. Brendlinger ${ }^{121}$, A.J. Brennan ${ }^{87}$, R. Brenner ${ }^{167}$, S. Bressler ${ }^{173}$, K. Bristow ${ }^{146 c}$, T.M. Bristow ${ }^{46}$, D. Britton ${ }^{53}$, F.M. Brochu ${ }^{28}$, I. Brock ${ }^{21}$, R. Brock ${ }^{89}$, C. Bromberg ${ }^{89}$, J. Bronner ${ }^{100}$, G. Brooijmans ${ }^{35}$, T. Brooks ${ }^{76}$, W.K. Brooks ${ }^{32 b}$, J. Brosamer ${ }^{15}$, E. Brost ${ }^{115}$, J. Brown ${ }^{55}$, P.A. Bruckman de Renstrom ${ }^{39}$, D. Bruncko ${ }^{145 b}$, R. Bruneliere ${ }^{48}$, S. Brunet ${ }^{60}$, A. Bruni ${ }^{20 a}$, G. Bruni ${ }^{20 a}$, M. Bruschi ${ }^{20 a}$, L. Bryngemark ${ }^{80}$, T. Buanes ${ }^{14}$, Q. Buat ${ }^{143}$, F. Bucci $^{49}$, P. Buchholz ${ }^{142}$, R.M. Buckingham ${ }^{119}$, A.G. Buckley ${ }^{53}$, S.I. Buda ${ }^{26 a}$, I.A. Budagov ${ }^{64}$, F. Buehrer ${ }^{48}$, L. Bugge ${ }^{118}$, M.K. Bugge ${ }^{118}$, O. Bulekov ${ }^{97}$, A.C. Bundock ${ }^{73}$, H. Burckhart ${ }^{30}$, S. Burdin ${ }^{73}$, B. Burghgrave ${ }^{107}$, S. Burke ${ }^{130}$, I. Burmeister ${ }^{43}$, E. Busato ${ }^{34}$, D. Büscher ${ }^{48}$, V. Büscher ${ }^{82}$, P. Bussey ${ }^{53}$, C.P. Buszello ${ }^{167}$, B. Butler ${ }^{57}$, J.M. Butler ${ }^{22}$, A.I. Butt ${ }^{3}$, C.M. Buttar ${ }^{53}$, J.M. Butterworth ${ }^{77}$, P. Butti ${ }^{106}$, W. Buttinger ${ }^{28}$, A. Buzatu ${ }^{53}$, M. Byszewski ${ }^{10}$, S. Cabrera Urbán ${ }^{168}$, D. Caforio ${ }^{20 a}, 20 b$, O. Cakir ${ }^{4 a}$, P. Calafiura ${ }^{15}$, A. Calandri ${ }^{137}$, G. Calderini ${ }^{79}$, P. Calfayan ${ }^{99}$, R. Calkins ${ }^{107}$, L.P. Caloba ${ }^{24 a}$, D. Calvet ${ }^{34}$, S. Calvet ${ }^{34}$, R. Camacho Toro ${ }^{49}$, S. Camarda ${ }^{42}$, D. Cameron ${ }^{118}$, L.M. Caminada ${ }^{15}$, R. Caminal Armadans ${ }^{12}$, S. Campana ${ }^{30}$, M. Campanelli ${ }^{77}$, A. Campoverde ${ }^{149}$, V. Canale ${ }^{103 a, 103 b}$, A. Canepa ${ }^{160 a}$, M. Cano Bret ${ }^{75}$, J. Cantero ${ }^{81}$, R. Cantrill ${ }^{125 a}$, T. Cao ${ }^{40}$, M.D.M. Capeans Garrido ${ }^{30}$, I. Caprini ${ }^{26 a}$, M. Caprini ${ }^{26 a}$, M. Capua ${ }^{37 a, 37 b}$, R. Caputo ${ }^{82}$, R. Cardarelli ${ }^{134 a}$, T. Carli $^{30}$, G. Carlino $^{103 a}$, L. Carminati $^{90 a, 90 b}$, S. Caron ${ }^{105}$, E. Carquin ${ }^{32 a}$, G.D. Carrillo-Montoya ${ }^{146 c}$, J.R. Carter ${ }^{28}$, J. Carvalho ${ }^{125 a, 125 c}$, D. Casadei ${ }^{77}$, M.P. Casado ${ }^{12}$, M. Casolino ${ }^{12}$, E. Castaneda-Miranda ${ }^{146 b}$, A. Castelli ${ }^{106}$, V. Castillo Gimenez ${ }^{168}$, N.F. Castro ${ }^{125 a}$, P. Catastini ${ }^{57}$, A. Catinaccio ${ }^{30}$, J.R. Catmore ${ }^{118}$, A. Cattai ${ }^{30}$, G. Cattani ${ }^{134 a, 134 b}$, S. Caughron ${ }^{89}$, V. Cavaliere ${ }^{166}$, D. Cavalli ${ }^{90 a}$, M. Cavalli-Sforza ${ }^{12}$, V. Cavasinni ${ }^{123 a, 123 b}$, F. Ceradini ${ }^{135 a, 135 b}$, B. Cerio $^{45}$, K. Cerny $^{128}$,

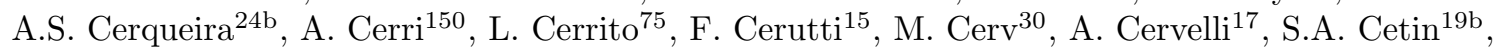
A. Chafaq ${ }^{136 a}$, D. Chakraborty ${ }^{107}$, I. Chalupkova ${ }^{128}$, P. Chang ${ }^{166}$, B. Chapleau ${ }^{86}$, J.D. Chapman ${ }^{28}$, D. Charfeddine ${ }^{116}$, D.G. Charlton ${ }^{18}$, C.C. Chau ${ }^{159}$, C.A. Chavez Barajas ${ }^{150}$, S. Cheatham ${ }^{86}$, A. Chegwidden ${ }^{89}$, S. Chekanov ${ }^{6}$, S.V. Chekulaev ${ }^{160 a}$, G.A. Chelkov ${ }^{64, f}$, M.A. Chelstowska ${ }^{88}$, C. Chen ${ }^{63}$, H. Chen ${ }^{25}$, K. Chen ${ }^{149}$, L. Chen ${ }^{33 d, g}$, S. Chen ${ }^{33 c}$, X. Chen ${ }^{146 c}$, Y. Chen ${ }^{66}$, Y. Chen ${ }^{35}$, H.C. Cheng ${ }^{88}$, Y. Cheng ${ }^{31}$, A. Cheplakov ${ }^{64}$, R. Cherkaoui El Moursli ${ }^{136 e}$, V. Chernyatin ${ }^{25, *}$, E. Cheu ${ }^{7}$, L. Chevalier ${ }^{137}$, V. Chiarella ${ }^{47}$, G. Chiefari103a,103b, J.T. Childers ${ }^{6}$, A. Chilingarov ${ }^{71}$, G. Chiodini ${ }^{72 a}$, A.S. Chisholm ${ }^{18}$, R.T. Chislett ${ }^{77}$, A. Chitan ${ }^{26 a}$, M.V. Chizhov ${ }^{64}$, S. Chouridou ${ }^{9}$, B.K.B. Chow $^{99}$, D. Chromek-Burckhart ${ }^{30}$, M.L. Chu ${ }^{152}$, J. Chudoba ${ }^{126}$, J.J. Chwastowski ${ }^{39}$, L. Chytka ${ }^{114}$, G. Ciapetti ${ }^{133 a, 133 b}$, A.K. Ciftci ${ }^{4 a}$, R. Ciftci ${ }^{4 a}$, D. Cinca ${ }^{53}$, V. Cindro ${ }^{74}$, A. Ciocio ${ }^{15}$, P. Cirkovic ${ }^{13 b}$, Z.H. Citron ${ }^{173}$, M. Citterio ${ }^{90 a}$,

M. Ciubancan ${ }^{26 a}$, A. Clark ${ }^{49}$, P.J. Clark ${ }^{46}$, R.N. Clarke ${ }^{15}$, W. Cleland ${ }^{124}$, J.C. Clemens ${ }^{84}$, C. Clement ${ }^{147 a, 147 b}$, Y. Coadou ${ }^{84}$, M. Cobal ${ }^{165 a, 165 c}$, A. Coccaro ${ }^{139}$, J. Cochran $^{63}$, L. Coffey $^{23}$, J.G. Cogan $^{144}$, J. Coggeshall ${ }^{166}$, B. Cole ${ }^{35}$, S. Cole ${ }^{107}$, A.P. Colijn ${ }^{106}$, J. Collot ${ }^{55}$, T. Colombo ${ }^{58 c}$, G. Colon ${ }^{85}$, G. Compostella ${ }^{100}$, P. Conde Muiño ${ }^{125 a, 125 b}$, E. Coniavitis ${ }^{48}$, M.C. Conidi $^{12}$, S.H. Connell ${ }^{146 b}$, I.A. Connelly ${ }^{76}$, S.M. Consonni ${ }^{90 a, 90 b}$, V. Consorti $^{48}$, S. Constantinescu ${ }^{26 a}$, C. Conta ${ }^{120 a, 120 b}$, G. Conti ${ }^{57}$, F. Conventi ${ }^{103 a, h}$, M. Cooke ${ }^{15}$, B.D. Cooper ${ }^{77}$,

A.M. Cooper-Sarkar ${ }^{119}$, N.J. Cooper-Smith ${ }^{76}$, K. Copic ${ }^{15}$, T. Cornelissen ${ }^{176}$, M. Corradi ${ }^{20 a}$,

F. Corriveau ${ }^{86, i}$, A. Corso-Radu ${ }^{164}$, A. Cortes-Gonzalez ${ }^{12}$, G. Cortiana ${ }^{100}$, G. Costa ${ }^{90 a}$, M.J. Costa ${ }^{168}$, D. Costanzo ${ }^{140}$, D. Côté ${ }^{8}$, G. Cottin $^{28}$, G. Cowan ${ }^{76}$, B.E. Cox ${ }^{83}$, K. Cranmer ${ }^{109}$, G. Cree ${ }^{29}$, S. Crépé-Renaudin ${ }^{55}$, F. Crescioli ${ }^{79}$, W.A. Cribbs ${ }^{147 a, 147 b}$, M. Crispin Ortuzar ${ }^{119}$ M. Cristinziani ${ }^{21}$, V. Croft ${ }^{105}$, G. Crosetti ${ }^{37 a, 37 b}$, C.-M. Cuciuc ${ }^{26 a}$, T. Cuhadar Donszelmann ${ }^{140}$, J. Cummings ${ }^{177}$, M. Curatolo ${ }^{47}$, C. Cuthbert ${ }^{151}$, H. Czirr ${ }^{142}$, P. Czodrowski ${ }^{3}$, Z. Czyczula ${ }^{177}$, S. D'Auria ${ }^{53}$, M. D'Onofrio ${ }^{73}$, M.J. Da Cunha Sargedas De Sousa ${ }^{125 a, 125 b}$, C. Da Via ${ }^{83}$, W. Dabrowski ${ }^{38 a}$, A. Dafinca ${ }^{119}$, T. Dai ${ }^{88}$, O. Dale ${ }^{14}$, F. Dallaire ${ }^{94}$, C. Dallapiccola ${ }^{85}$, M. Dam ${ }^{36}$, A.C. Daniells ${ }^{18}$, M. Dano Hoffmann ${ }^{137}$, V. Dao ${ }^{48}$, G. Darbo ${ }^{50 a}$, S. Darmora ${ }^{8}$, J.A. Dassoulas ${ }^{42}$,

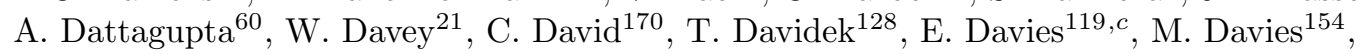




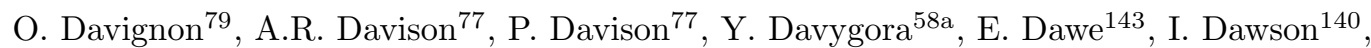
R.K. Daya-Ishmukhametova ${ }^{85}$, K. De ${ }^{8}$, R. de Asmundis ${ }^{103 a}$, S. De Castro ${ }^{20 a, 20 b}$, S. De Cecco ${ }^{79}$, N. De Groot ${ }^{105}$, P. de Jong ${ }^{106}$, H. De la Torre ${ }^{81}$, F. De Lorenzi ${ }^{63}$, L. De Nooij ${ }^{106}$, D. De Pedis ${ }^{133 a}$, A. De Salvo ${ }^{133 a}$, U. De Sanctis ${ }^{165 a, 165 b}$, A. De Santo ${ }^{150}$, J.B. De Vivie De Regie ${ }^{116}$, W.J. Dearnaley ${ }^{71}$, R. Debbe ${ }^{25}$, C. Debenedetti ${ }^{138}$, B. Dechenaux ${ }^{55}$, D.V. Dedovich ${ }^{64}$, I. Deigaard ${ }^{106}$, J. Del Peso ${ }^{81}$, T. Del Prete ${ }^{123 a, 123 b}$, F. Deliot ${ }^{137}$, C.M. Delitzsch ${ }^{49}$, M. Deliyergiyev ${ }^{74}$, A. Dell'Acqua ${ }^{30}$, L. Dell'Asta ${ }^{22}$, M. Dell'Orso ${ }^{123 a, 123 b}$, M. Della Pietra ${ }^{103 a, h}$, D. della Volpe ${ }^{49}$, M. Delmastro ${ }^{5}$, P.A. Delsart ${ }^{55}$, C. Deluca ${ }^{106}$, S. Demers ${ }^{177}$, M. Demichev ${ }^{64}$, A. Demilly ${ }^{79}$, S.P. Denisov ${ }^{129}$, D. Derendarz ${ }^{39}$, J.E. Derkaoui ${ }^{136 d}$, F. Derue ${ }^{79}$, P. Dervan ${ }^{73}$, K. $\operatorname{Desch}^{21}$, C. Deterre ${ }^{42}$, P.O. Deviveiros ${ }^{106}$, A. Dewhurst ${ }^{130}$, S. Dhaliwal ${ }^{106}$,

A. Di Ciaccio ${ }^{134 a, 134 b}$, L. Di Ciaccio ${ }^{5}$, A. Di Domenico ${ }^{133 a, 133 b}$, C. Di Donato 103a,103b, A. Di Girolamo ${ }^{30}$, B. Di Girolamo ${ }^{30}$, A. Di Mattia ${ }^{153}$, B. Di Micco ${ }^{135 a, 135 b}$, R. Di Nardo ${ }^{47}$,

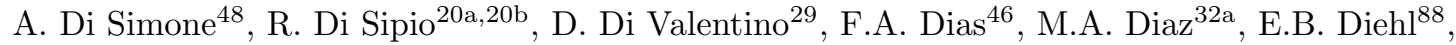
J. Dietrich ${ }^{42}$, T.A. Dietzsch ${ }^{58 a}$, S. Diglio ${ }^{84}$, A. Dimitrievska $^{13 a}$, J. Dingfelder $^{21}$,

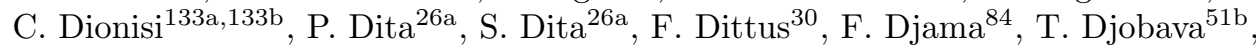
M.A.B. do Vale ${ }^{24 c}$, A. Do Valle Wemans ${ }^{125 a, 125 g}$, T.K.O. Doan ${ }^{5}$, D. Dobos ${ }^{30}$, C. Doglioni ${ }^{49}$, T. Doherty ${ }^{53}$, T. Dohmae ${ }^{156}$, J. Dolejsi $^{128}$, Z. Dolezal ${ }^{128}$, B.A. Dolgoshein ${ }^{97, *}$, M. Donadelli ${ }^{24 \mathrm{~d}}$, S. Donati ${ }^{123 a, 123 b}$, P. Dondero ${ }^{120 a, 120 b}$, J. Donini ${ }^{34}$, J. Dopke ${ }^{130}$, A. Doria ${ }^{103 a}$, M.T. Dova ${ }^{70}$, A.T. Doyle ${ }^{53}$, M. Dris ${ }^{10}$, J. Dubbert ${ }^{88}$, S. Dube ${ }^{15}$, E. Dubreuil ${ }^{34}$, E. Duchovni ${ }^{173}$, G. Duckeck ${ }^{99}$,

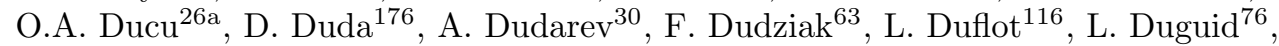
M. Dührssen ${ }^{30}$, M. Dunford ${ }^{58 a}$, H. Duran Yildiz ${ }^{4 a}$, M. Düren ${ }^{52}$, A. Durglishvili ${ }^{51 b}$, M. Dwuznik ${ }^{38 a}$, M. Dyndal ${ }^{38 a}$, J. Ebke ${ }^{99}$, W. Edson ${ }^{2}$, N.C. Edwards ${ }^{46}$, W. Ehrenfeld ${ }^{21}$, T. Eifert ${ }^{144}$, G. Eigen ${ }^{14}$, K. Einsweiler ${ }^{15}$, T. Ekelof ${ }^{167}$, M. El Kacimi ${ }^{136 c}$, M. Ellert ${ }^{167}$, S. Elles ${ }^{5}$, F. Ellinghaus ${ }^{82}$, N. Ellis ${ }^{30}$, J. Elmsheuser ${ }^{99}$, M. Elsing ${ }^{30}$, D. Emeliyanov ${ }^{130}$, Y. Enari ${ }^{156}$, O.C. Endner ${ }^{82}$, M. Endo ${ }^{117}$, R. Engelmann ${ }^{149}$, J. Erdmann ${ }^{177}$, A. Ereditato ${ }^{17}$, D. Eriksson ${ }^{147 a}$, G. Ernis ${ }^{176}$, J. Ernst ${ }^{2}$, M. Ernst ${ }^{25}$, J. Ernwein ${ }^{137}$, D. Errede ${ }^{166}$, S. Errede ${ }^{166}$, E. Ertel ${ }^{82}$, M. Escalier ${ }^{116}$, H. Esch $^{43}$, C. Escobar ${ }^{124}$, B. Esposito ${ }^{47}$, A.I. Etienvre ${ }^{137}$, E. Etzion ${ }^{154}$, H. Evans ${ }^{60}$, A. Ezhilov ${ }^{122}$, L. Fabbri ${ }^{20 a, 20 b}$, G. Facini ${ }^{31}$, R.M. Fakhrutdinov ${ }^{129}$, S. Falciano ${ }^{133 a}$, R.J. Falla ${ }^{77}$, J. Faltova ${ }^{128}$, Y. Fang ${ }^{33 a}$, M. Fanti ${ }^{90 a, 90 b}$, A. Farbin ${ }^{8}$, A. Farilla ${ }^{135 a}$, T. Farooque ${ }^{12}$, S. Farrell ${ }^{15}$, S.M. Farrington ${ }^{171}$, P. Farthouat ${ }^{30}$, F. Fassi ${ }^{136 e}$, P. Fassnacht ${ }^{30}$, D. Fassouliotis ${ }^{9}$, A. Favareto ${ }^{50 a, 50 b}$, L. Fayard ${ }^{116}$, P. Federic ${ }^{145 a}$, O.L. Fedin ${ }^{122, j}$, W. Fedorko ${ }^{169}$,

M. Fehling-Kaschek ${ }^{48}$, S. Feigl ${ }^{30}$, L. Feligioni ${ }^{84}$, C. Feng ${ }^{33 d}$, E.J. Feng ${ }^{6}$, H. Feng ${ }^{88}$,

A.B. Fenyuk ${ }^{129}$, S. Fernandez Perez $^{30}$, S. Ferrag ${ }^{53}$, J. Ferrando ${ }^{53}$, A. Ferrari ${ }^{167}$, P. Ferrari ${ }^{106}$, R. Ferrari ${ }^{120 a}$, D.E. Ferreira de Lima $^{53}$, A. Ferrer ${ }^{168}$, D. Ferrere ${ }^{49}$, C. Ferretti ${ }^{88}$,

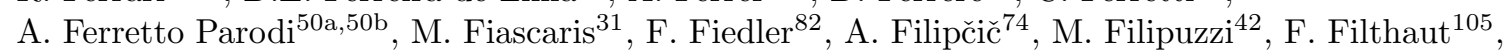
M. Fincke-Keeler ${ }^{170}$, K.D. Finelli ${ }^{151}$, M.C.N. Fiolhais ${ }^{125 a, 125 c}$, L. Fiorini ${ }^{168}$, A. Firan ${ }^{40}$, A. Fischer ${ }^{2}$, J. Fischer ${ }^{176}$, W.C. Fisher ${ }^{89}$, E.A. Fitzgerald ${ }^{23}$, M. Flechl ${ }^{48}$, I. Fleck ${ }^{142}$, P. Fleischmann ${ }^{88}$, S. Fleischmann ${ }^{176}$, G.T. Fletcher ${ }^{140}$, G. Fletcher ${ }^{75}$, T. Flick ${ }^{176}$, A. Floderus ${ }^{80}$, L.R. Flores Castillo ${ }^{174, k}$, A.C. Florez Bustos ${ }^{160 b}$, M.J. Flowerdew ${ }^{100}$, A. Formica ${ }^{137}$, A. Forti ${ }^{83}$, D. Fortin ${ }^{160 a}$, D. Fournier ${ }^{116}$, H. Fox ${ }^{71}$, S. Fracchia ${ }^{12}$, P. Francavilla ${ }^{79}$, M. Franchini $20 a, 20 b$, S. Franchino ${ }^{30}$, D. Francis ${ }^{30}$, L. Franconi ${ }^{118}$, M. Franklin ${ }^{57}$, S. Franz ${ }^{61}$, M. Fraternali ${ }^{120 a, 120 b}$, S.T. French ${ }^{28}$, C. Friedrich ${ }^{42}$, F. Friedrich ${ }^{44}$, D. Froidevaux ${ }^{30}$, J.A. Frost ${ }^{28}$, C. Fukunaga ${ }^{157}$, E. Fullana Torregrosa ${ }^{82}$, B.G. Fulsom ${ }^{144}$, J. Fuster ${ }^{168}$, C. Gabaldon ${ }^{55}$, O. Gabizon ${ }^{173}$,

A. Gabrielli20a,20b, A. Gabrielli ${ }^{133 a, 133 b}$, S. Gadatsch ${ }^{106}$, S. Gadomski ${ }^{49}$, G. Gagliardi ${ }^{50 a, 50 b}$, P. Gagnon ${ }^{60}$, C. Galea ${ }^{105}$, B. Galhardo ${ }^{125 a, 125 c}$, E.J. Gallas ${ }^{119}$, V. Gallo ${ }^{17}$, B.J. Gallop ${ }^{130}$, P. Gallus ${ }^{127}$, G. Galster ${ }^{36}$, K.K. Gan ${ }^{110}$, R.P. Gandrajula ${ }^{62}$, J. Gao ${ }^{33 b, g}$, Y.S. Gao ${ }^{144, e}$,

F.M. Garay Walls ${ }^{46}$, F. Garberson ${ }^{177}$, C. García ${ }^{168}$, J.E. García Navarro ${ }^{168}$, M. Garcia-Sciveres ${ }^{15}$, R.W. Gardner ${ }^{31}$, N. Garelli ${ }^{144}$, V. Garonne ${ }^{30}$, C. Gatti ${ }^{47}$, G. Gaudio ${ }^{120 a}$, B. Gaur ${ }^{142}$,

L. Gauthier ${ }^{94}$, P. Gauzzi ${ }^{133 a, 133 b}$, I.L. Gavrilenko ${ }^{95}$, C. Gay ${ }^{169}$, G. Gaycken ${ }^{21}$, E.N. Gazis ${ }^{10}$, P. Ge ${ }^{33 d}$, Z. Gecse ${ }^{169}$, C.N.P. Gee ${ }^{130}$, D.A.A. Geerts ${ }^{106}$, Ch. Geich-Gimbel ${ }^{21}$,

K. Gellerstedt ${ }^{147 a, 147 b}$, C. Gemme ${ }^{50 a}$, A. Gemmell ${ }^{53}$, M.H. Genest ${ }^{55}$, S. Gentile ${ }^{133 a, 133 b}$, M. George ${ }^{54}$, S. George ${ }^{76}$, D. Gerbaudo ${ }^{164}$, A. Gershon $^{154}$, H. Ghazlane ${ }^{136 b}$, N. Ghodbane ${ }^{34}$, 
B. Giacobbe ${ }^{20 a}$, S. Giagu ${ }^{133 a, 133 b}$, V. Giangiobbe ${ }^{12}$, P. Giannetti ${ }^{123 a, 123 b}$, F. Gianotti ${ }^{30}$, B. Gibbard ${ }^{25}$, S.M. Gibson ${ }^{76}$, M. Gilchriese ${ }^{15}$, T.P.S. Gillam ${ }^{28}$, D. Gillberg ${ }^{30}$, G. Gilles ${ }^{34}$, D.M. Gingrich ${ }^{3, d}$, N. Giokaris ${ }^{9}$, M.P. Giordani ${ }^{165 a, 165 c}$, R. Giordano ${ }^{103 a, 103 b}$, F.M. Giorgi ${ }^{20 a}$, F.M. Giorgi ${ }^{16}$, P.F. Giraud ${ }^{137}$, D. Giugni ${ }^{90 a}$, C. Giuliani ${ }^{48}$, M. Giulini ${ }^{58 b}$, B.K. Gjelsten ${ }^{118}$, S. Gkaitatzis ${ }^{155}$, I. Gkialas ${ }^{155, l}$, L.K. Gladilin ${ }^{98}$, C. Glasman ${ }^{81}$, J. Glatzer ${ }^{30}$, P.C.F. Glaysher ${ }^{46}$,

A. Glazov ${ }^{42}$, G.L. Glonti ${ }^{64}$, M. Goblirsch-Kolb ${ }^{100}$, J.R. Goddard ${ }^{75}$, J. Godfrey ${ }^{143}$, J. Godlewski ${ }^{30}$, C. Goeringer ${ }^{82}$, S. Goldfarb ${ }^{88}$, T. Golling ${ }^{177}$, D. Golubkov ${ }^{129}$, A. Gomes ${ }^{125 a, 125 b, 125 d}$,

L.S. Gomez Fajardo ${ }^{42}$, R. Gonçalo ${ }^{125 a}$, J. Goncalves Pinto Firmino Da Costa ${ }^{137}$, L. Gonella ${ }^{21}$, S. González de la Hoz ${ }^{168}$, G. Gonzalez Parra ${ }^{12}$, S. Gonzalez-Sevilla ${ }^{49}$, L. Goossens ${ }^{30}$, P.A. Gorbounov ${ }^{96}$, H.A. Gordon ${ }^{25}$, I. Gorelov ${ }^{104}$, B. Gorini ${ }^{30}$, E. Gorini ${ }^{72 a, 72 b}$, A. Gorišek ${ }^{74}$ E. Gornicki ${ }^{39}$, A.T. Goshaw ${ }^{6}$, C. Gössling ${ }^{43}$, M.I. Gostkin ${ }^{64}$, M. Gouighri ${ }^{136 a}$, D. Goujdami ${ }^{136 c}$, M.P. Goulette ${ }^{49}$, A.G. Goussiou ${ }^{139}$, C. Goy ${ }^{5}$, S. Gozpinar ${ }^{23}$, H.M.X. Grabas ${ }^{137}$, L. Graber ${ }^{54}$, I. Grabowska-Bold ${ }^{38 a}$, P. Grafström ${ }^{20 a, 20 b}$, K-J. Grahn ${ }^{42}$, J. Gramling ${ }^{49}$, E. Gramstad ${ }^{118}$, S. Grancagnolo ${ }^{16}$, V. Grassi ${ }^{149}$, V. Gratchev ${ }^{122}$, H.M. Gray ${ }^{30}$, E. Graziani ${ }^{135 a}$, O.G. Grebenyuk ${ }^{122}$, Z.D. Greenwood ${ }^{78, m}$, K. Gregersen ${ }^{77}$, I.M. Gregor ${ }^{42}$, P. Grenier ${ }^{144}$, J. Griffiths ${ }^{8}$, A.A. Grillo ${ }^{138}$, K. Grimm ${ }^{71}$, S. Grinstein ${ }^{12, n}$, Ph. Gris ${ }^{34}$, Y.V. Grishkevich ${ }^{98}$, J.-F. Grivaz ${ }^{116}$, J.P. Grohs ${ }^{44}$, A. Grohsjean ${ }^{42}$, E. Gross ${ }^{173}$, J. Grosse-Knetter ${ }^{54}$,

G.C. Grossi ${ }^{134 a, 134 b}$, J. Groth-Jensen ${ }^{173}$, Z.J. Grout ${ }^{150}$, L. Guan ${ }^{33 b}$, F. Guescini ${ }^{49}$, D. Guest ${ }^{177}$, O. Gueta ${ }^{154}$, C. Guicheney ${ }^{34}$, E. Guido $50 \mathrm{a}, 50 \mathrm{~b}$, T. Guillemin ${ }^{116}$, S. Guindon ${ }^{2}$, U. Gul ${ }^{53}$,

C. Gumpert ${ }^{44}$, J. Gunther ${ }^{127}$, J. Guo ${ }^{35}$, S. Gupta ${ }^{119}$, P. Gutierrez ${ }^{112}$, N.G. Gutierrez Ortiz ${ }^{53}$, C. Gutschow ${ }^{77}$, N. Guttman ${ }^{154}$, C. Guyot ${ }^{137}$, C. Gwenlan ${ }^{119}$, C.B. Gwilliam ${ }^{73}$, A. Haas ${ }^{109}$,

C. Haber ${ }^{15}$, H.K. Hadavand ${ }^{8}$, N. Haddad ${ }^{136 e}$, P. Haefner ${ }^{21}$, S. Hageböck ${ }^{21}$, Z. Hajduk ${ }^{39}$, H. Hakobyan ${ }^{178}$, M. Haleem ${ }^{42}$, D. Hall ${ }^{119}$, G. Halladjian ${ }^{89}$, K. Hamacher ${ }^{176}$, P. Hamal ${ }^{114}$, K. Hamano ${ }^{170}$, M. Hamer ${ }^{54}$, A. Hamilton ${ }^{146 a}$, S. Hamilton ${ }^{162}$, G.N. Hamity ${ }^{146 c}$, P.G. Hamnett ${ }^{42}$, L. $\operatorname{Han}^{33 \mathrm{~b}}$, K. Hanagaki ${ }^{117}$, K. Hanawa ${ }^{156}$, M. Hance ${ }^{15}$, P. Hanke ${ }^{58 a}$, R. Hanna ${ }^{137}$, J.B. Hansen ${ }^{36}$, J.D. Hansen ${ }^{36}$, P.H. Hansen ${ }^{36}$, K. Hara ${ }^{161}$, A.S. Hard ${ }^{174}$, T. Harenberg ${ }^{176}$, F. Hariri ${ }^{116}$,

S. Harkusha ${ }^{91}$, D. Harper ${ }^{88}$, R.D. Harrington ${ }^{46}$, O.M. Harris ${ }^{139}$, P.F. Harrison ${ }^{171}$, F. Hartjes ${ }^{106}$, M. Hasegawa ${ }^{66}$, S. Hasegawa ${ }^{102}$, Y. Hasegawa ${ }^{141}$, A. Hasib ${ }^{112}$, S. Hassani ${ }^{137}$, S. Haug ${ }^{17}$

M. Hauschild ${ }^{30}$, R. Hauser ${ }^{89}$, M. Havranek ${ }^{126}$, C.M. Hawkes ${ }^{18}$, R.J. Hawkings ${ }^{30}$, A.D. Hawkins ${ }^{80}$,

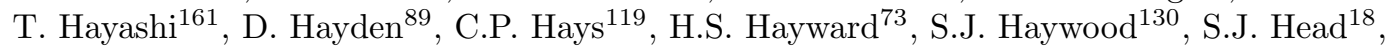

T. Heck ${ }^{82}$, V. Hedberg ${ }^{80}$, L. Heelan ${ }^{8}$, S. Heim ${ }^{121}$, T. Heim ${ }^{176}$, B. Heinemann ${ }^{15}$, L. Heinrich ${ }^{109}$,

J. Hejbal ${ }^{126}$, L. Helary ${ }^{22}$, C. Heller ${ }^{99}$, M. Heller ${ }^{30}$, S. Hellman ${ }^{147 a, 147 b}$, D. Hellmich ${ }^{21}$,

C. Helsens ${ }^{30}$, J. Henderson ${ }^{119}$, R.C.W. Henderson ${ }^{71}$, Y. Heng ${ }^{174}$, C. Hengler ${ }^{42}$, A. Henrichs ${ }^{177}$, A.M. Henriques Correia ${ }^{30}$, S. Henrot-Versille ${ }^{116}$, C. Hensel ${ }^{54}$, G.H. Herbert ${ }^{16}$,

Y. Hernández Jiménez ${ }^{168}$, R. Herrberg-Schubert ${ }^{16}$, G. Herten ${ }^{48}$, R. Hertenberger ${ }^{99}$, L. Hervas ${ }^{30}$, G.G. Hesketh ${ }^{77}$, N.P. Hessey ${ }^{106}$, R. Hickling ${ }^{75}$, E. Higón-Rodriguez ${ }^{168}$, E. Hill ${ }^{170}$, J.C. Hill ${ }^{28}$, K.H. Hiller ${ }^{42}$, S. Hillert ${ }^{21}$, S.J. Hillier ${ }^{18}$, I. Hinchliffe ${ }^{15}$, E. Hines ${ }^{121}$, M. Hirose ${ }^{158}$,

D. Hirschbuehl ${ }^{176}$, J. Hobbs ${ }^{149}$, N. Hod ${ }^{106}$, M.C. Hodgkinson ${ }^{140}$, P. Hodgson ${ }^{140}$, A. Hoecker ${ }^{30}$, M.R. Hoeferkamp ${ }^{104}$, F. Hoenig ${ }^{99}$, J. Hoffman ${ }^{40}$, D. Hoffmann ${ }^{84}$, J.I. Hofmann ${ }^{58 a}$, M. Hohlfeld ${ }^{82}$, T.R. Holmes ${ }^{15}$, T.M. Hong ${ }^{121}$, L. Hooft van Huysduynen ${ }^{109}$, J-Y. Hostachy ${ }^{55}$, S. Hou ${ }^{152}$,

A. Hoummada ${ }^{136 a}$, J. Howard ${ }^{119}$, J. Howarth ${ }^{42}$, M. Hrabovsky ${ }^{114}$, I. Hristova ${ }^{16}$, J. Hrivnac ${ }^{116}$,

T. Hryn'ova ${ }^{5}$, C. Hsu ${ }^{146 c}$, P.J. Hsu ${ }^{82}$, S.-C. Hsu ${ }^{139}$, D. Hu ${ }^{35}$, X. Hu ${ }^{25}$, Y. Huang ${ }^{42}$, Z. Hubacek ${ }^{30}$, F. Hubaut ${ }^{84}$, F. Huegging ${ }^{21}$, T.B. Huffman ${ }^{119}$, E.W. Hughes ${ }^{35}$, G. Hughes ${ }^{71}$, M. Huhtinen ${ }^{30}$,

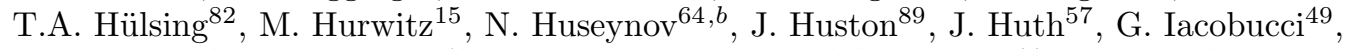
G. Iakovidis $^{10}$, I. Ibragimov ${ }^{142}$, L. Iconomidou-Fayard ${ }^{116}$, E. Ideal ${ }^{177}$, P. Iengo ${ }^{103 a}$, O. Igonkina ${ }^{106}$, T. Iizawa ${ }^{172}$, Y. Ikegami ${ }^{65}$, K. Ikematsu ${ }^{142}$, M. Ikeno ${ }^{65}$, Y. Ilchenko ${ }^{31, o}$, D. Iliadis ${ }^{155}$, N. Ilic ${ }^{159}$,

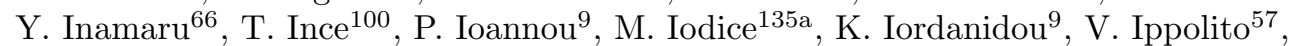

A. Irles Quiles ${ }^{168}$, C. Isaksson ${ }^{167}$, M. Ishino ${ }^{67}$, M. Ishitsuka ${ }^{158}$, R. Ishmukhametov ${ }^{110}$,

C. Issever ${ }^{119}$, S. Istin ${ }^{19 a}$, J.M. Iturbe Ponce ${ }^{83}$, R. Iuppa ${ }^{134 a, 134 b}$, J. Ivarsson ${ }^{80}$, W. Iwanski ${ }^{39}$,

H. Iwasaki ${ }^{65}$, J.M. Izen ${ }^{41}$, V. Izzo ${ }^{103 a}$, B. Jackson ${ }^{121}$, M. Jackson ${ }^{73}$, P. Jackson ${ }^{1}$, M.R. Jaekel ${ }^{30}$,

V. Jain ${ }^{2}$, K. Jakobs ${ }^{48}$, S. Jakobsen ${ }^{30}$, T. Jakoubek ${ }^{126}$, J. Jakubek ${ }^{127}$, D.O. Jamin ${ }^{152}$, D.K. Jana ${ }^{78}$, E. Jansen ${ }^{77}$, H. Jansen ${ }^{30}$, J. Janssen ${ }^{21}$, M. Janus ${ }^{171}$, G. Jarlskog ${ }^{80}$, N. Javadov ${ }^{64, b}$, T. Javưrek ${ }^{48}$, 
L. Jeanty ${ }^{15}$, J. Jejelava ${ }^{51 a, p}$, G.-Y. Jeng ${ }^{151}$, D. Jennens ${ }^{87}$, P. Jenni ${ }^{48, q}$, J. Jentzsch ${ }^{43}$, C. Jeske ${ }^{171}$, S. Jézéquel ${ }^{5}$, H. Ji ${ }^{174}$, J. Jia ${ }^{149}$, Y. Jiang ${ }^{33 b}$, M. Jimenez Belenguer ${ }^{42}$, S. Jin ${ }^{33 a}$, A. Jinaru ${ }^{26 a}$, O. Jinnouchi ${ }^{158}$, M.D. Joergensen ${ }^{36}$, K.E. Johansson ${ }^{147 a, 147 b}$, P. Johansson ${ }^{140}$, K.A. Johns ${ }^{7}$, K. Jon-And ${ }^{147 a, 147 b}$, G. Jones ${ }^{171}$, R.W.L. Jones ${ }^{71}$, T.J. Jones ${ }^{73}$, J. Jongmanns ${ }^{58 a}$, P.M. Jorge ${ }^{125 a, 125 b}$, K.D. Joshi ${ }^{83}$, J. Jovicevic ${ }^{148}$, X. Ju ${ }^{174}$, C.A. Jung ${ }^{43}$, R.M. Jungst ${ }^{30}$, P. Jussel ${ }^{61}$, A. Juste Rozas ${ }^{12, n}$, M. Kaci ${ }^{168}$, A. Kaczmarska ${ }^{39}$, M. Kado ${ }^{116}$, H. Kagan ${ }^{110}$, M. Kagan ${ }^{144}$, E. Kajomovitz ${ }^{45}$, C.W. Kalderon ${ }^{119}$, S. Kama ${ }^{40}$, A. Kamenshchikov ${ }^{129}$ N. Kanaya ${ }^{156}$, M. Kaneda ${ }^{30}$, S. Kaneti ${ }^{28}$, V.A. Kantserov ${ }^{97}$, J. Kanzaki ${ }^{65}$, B. Kaplan ${ }^{109}$, A. Kapliy ${ }^{31}$, D. $\operatorname{Kar}^{53}$, K. Karakostas ${ }^{10}$, N. Karastathis ${ }^{10}$, M. Karnevskiy ${ }^{82}$, S.N. Karpov ${ }^{64}$, Z.M. Karpova ${ }^{64}$, K. Karthik ${ }^{109}$, V. Kartvelishvili ${ }^{71}$, A.N. Karyukhin ${ }^{129}$, L. Kashif ${ }^{174}$, G. Kasieczka ${ }^{58 b}$, R.D. Kass ${ }^{110}$, A. Kastanas ${ }^{14}$, Y. Kataoka ${ }^{156}$, A. Katre ${ }^{49}$, J. Katzy ${ }^{42}$, V. Kaushik ${ }^{7}$, K. Kawagoe ${ }^{69}$, T. Kawamoto ${ }^{156}$, G. Kawamura ${ }^{54}$, S. Kazama ${ }^{156}$, V.F. Kazanin ${ }^{108}$, M.Y. Kazarinov ${ }^{64}$, R. Keeler ${ }^{170}$, R. Kehoe ${ }^{40}$, M. Keil ${ }^{54}$, J.S. Keller ${ }^{42}$, J.J. Kempster ${ }^{76}$,

H. Keoshkerian ${ }^{5}$, O. Kepka ${ }^{126}$, B.P. Kerševan ${ }^{74}$, S. Kersten ${ }^{176}$, K. Kessoku ${ }^{156}$, J. Keung ${ }^{159}$, F. Khalil-zada ${ }^{11}$, H. Khandanyan ${ }^{147 a, 147 b}$, A. Khanov ${ }^{113}$, A. Khodinov ${ }^{97}$, A. Khomich ${ }^{58 a}$, T.J. Khoo ${ }^{28}$, G. Khoriauli ${ }^{21}$, A. Khoroshilov ${ }^{176}$, V. Khovanskiy ${ }^{96}$, E. Khramov ${ }^{64}$, J. Khubua ${ }^{51 b}$, H.Y. Kim ${ }^{8}$, H. Kim ${ }^{147 a, 147 b}$, S.H. Kim ${ }^{161}$, N. Kimura ${ }^{172}$, O. Kind ${ }^{16}$, B.T. King ${ }^{73}$, M. King ${ }^{168}$, R.S.B. King ${ }^{119}$, S.B. King ${ }^{169}$, J. Kirk ${ }^{130}$, A.E. Kiryunin ${ }^{100}$, T. Kishimoto ${ }^{66}$, D. Kisielewska ${ }^{38 a}$, F. Kiss ${ }^{48}$, T. Kittelmann ${ }^{124}$, K. Kiuchi ${ }^{161}$, E. Kladiva ${ }^{145 b}$, M. Klein ${ }^{73}$, U. Klein ${ }^{73}$,

K. Kleinknecht ${ }^{82}$, P. Klimek ${ }^{147 a, 147 b}$, A. Klimentov ${ }^{25}$, R. Klingenberg ${ }^{43}$, J.A. Klinger ${ }^{83}$, T. Klioutchnikova ${ }^{30}$, P.F. Klok ${ }^{105}$, E.-E. Kluge ${ }^{58 a}$, P. Kluit ${ }^{106}$, S. Kluth ${ }^{100}$, E. Kneringer ${ }^{61}$, E.B.F.G. Knoops ${ }^{84}$, A. Knue ${ }^{53}$, D. Kobayashi ${ }^{158}$, T. Kobayashi ${ }^{156}$, M. Kobel ${ }^{44}$, M. Kocian ${ }^{144}$, P. Kodys ${ }^{128}$, P. Koevesarki ${ }^{21}$, T. Koffas ${ }^{29}$, E. Koffeman ${ }^{106}$, L.A. Kogan ${ }^{119}$, S. Kohlmann ${ }^{176}$, Z. Kohout ${ }^{127}$, T. Kohriki ${ }^{65}$, T. Koi ${ }^{144}$, H. Kolanoski ${ }^{16}$, I. Koletsou ${ }^{5}$, J. Koll ${ }^{89}$, A.A. Komar ${ }^{95, *}$, Y. Komori ${ }^{156}$, T. Kondo ${ }^{65}$, N. Kondrashova ${ }^{42}$, K. Köneke ${ }^{48}$, A.C. König ${ }^{105}$, S. König ${ }^{82}$, T. Kono ${ }^{65, r}$, R. Konoplich ${ }^{109, s}$, N. Konstantinidis ${ }^{77}$, R. Kopeliansky ${ }^{153}$, S. Koperny ${ }^{38 a}$,

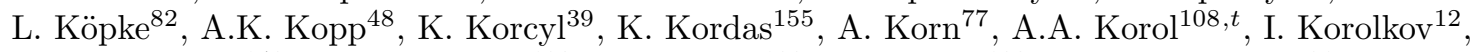
E.V. Korolkova ${ }^{140}$, V.A. Korotkov ${ }^{129}$, O. Kortner ${ }^{100}$, S. Kortner ${ }^{100}$, V.V. Kostyukhin ${ }^{21}$, V.M. Kotov ${ }^{64}$, A. Kotwal ${ }^{45}$, C. Kourkoumelis ${ }^{9}$, V. Kouskoura ${ }^{155}$, A. Koutsman ${ }^{160 a}$, R. Kowalewski ${ }^{170}$, T.Z. Kowalski ${ }^{38 a}$, W. Kozanecki ${ }^{137}$, A.S. Kozhin ${ }^{129}$, V. Kral ${ }^{127}$,

V.A. Kramarenko ${ }^{98}$, G. Kramberger ${ }^{74}$, D. Krasnopevtsev ${ }^{97}$, M.W. Krasny ${ }^{79}$, A. Krasznahorkay ${ }^{30}$, J.K. Kraus ${ }^{21}$, A. Kravchenko ${ }^{25}$, S. Kreiss ${ }^{109}$, M. Kretz ${ }^{58 c}$, J. Kretzschmar ${ }^{73}$, K. Kreutzfeldt ${ }^{52}$, P. Krieger ${ }^{159}$, K. Kroeninger ${ }^{54}$, H. Kroha ${ }^{100}$, J. Kroll ${ }^{121}$, J. Kroseberg ${ }^{21}$, J. Krstic ${ }^{13 a}$, U. Kruchonak ${ }^{64}$, H. Krüger ${ }^{21}$, T. Kruker ${ }^{17}$, N. Krumnack ${ }^{63}$, Z.V. Krumshteyn ${ }^{64}$, A. Kruse ${ }^{174}$, M.C. Kruse ${ }^{45}$, M. Kruskal ${ }^{22}$, T. Kubota ${ }^{87}$, S. Kuday ${ }^{4 a}$, S. Kuehn ${ }^{48}$, A. Kugel ${ }^{58 c}$, A. Kuhl ${ }^{138}$, T. Kuhl ${ }^{42}$, V. Kukhtin ${ }^{64}$, Y. Kulchitsky ${ }^{91}$, S. Kuleshov ${ }^{32 b}$, M. Kuna ${ }^{133 a, 133 b}$, J. Kunkle ${ }^{121}$, A. Kupco ${ }^{126}$, H. Kurashige ${ }^{66}$, Y.A. Kurochkin ${ }^{91}$, R. Kurumida ${ }^{66}$, V. Kus ${ }^{126}$, E.S. Kuwertz ${ }^{148}$, M. Kuze ${ }^{158}$, J. Kvita ${ }^{114}$, A. La Rosa ${ }^{49}$, L. La Rotonda ${ }^{37 a, 37 b}$, C. Lacasta ${ }^{168}$, F. Lacava ${ }^{133 a, 133 b}$, J. Lacey ${ }^{29}$, H. Lacker ${ }^{16}$, D. Lacour ${ }^{79}$, V.R. Lacuesta ${ }^{168}$, E. Ladygin ${ }^{64}$, R. Lafaye ${ }^{5}$, B. Laforge ${ }^{79}$, T. Lagouri ${ }^{177}$, S. Lai ${ }^{48}$, H. Laier ${ }^{58 a}$, L. Lambourne ${ }^{77}$, S. Lammers ${ }^{60}$, C.L. Lampen ${ }^{7}$, W. Lampl ${ }^{7}$, E. Lançon ${ }^{137}$, U. Landgraf ${ }^{48}$, M.P.J. Landon ${ }^{75}$, V.S. Lang ${ }^{58 a}$, A.J. Lankford ${ }^{164}$, F. Lanni ${ }^{25}$,

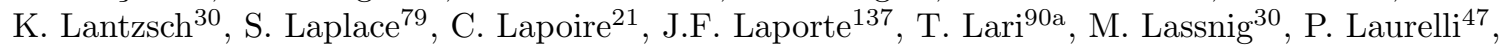
W. Lavrijsen ${ }^{15}$, A.T. Law ${ }^{138}$, P. Laycock ${ }^{73}$, O. Le Dortz ${ }^{79}$, E. Le Guirriec ${ }^{84}$, E. Le Menedeu ${ }^{12}$, T. LeCompte ${ }^{6}$, F. Ledroit-Guillon ${ }^{55}$, C.A. Lee ${ }^{152}$, H. Lee ${ }^{106}$, J.S.H. Lee ${ }^{117}$, S.C. Lee ${ }^{152}$, L. Lee ${ }^{177}$, G. Lefebvre ${ }^{79}$, M. Lefebvre ${ }^{170}$, F. Legger ${ }^{99}$, C. Leggett ${ }^{15}$, A. Lehan ${ }^{73}$, M. Lehmacher ${ }^{21}$, G. Lehmann Miotto ${ }^{30}$, X. Lei ${ }^{7}$, W.A. Leight ${ }^{29}$, A. Leisos ${ }^{155}$, A.G. Leister ${ }^{177}$, M.A.L. Leite ${ }^{24 d}$, R. Leitner ${ }^{128}$, D. Lellouch ${ }^{173}$, B. Lemmer ${ }^{54}$, K.J.C. Leney ${ }^{77}$, T. Lenz ${ }^{21}$, G. Lenzen ${ }^{176}$, B. Lenzi ${ }^{30}$, R. Leone ${ }^{7}$, S. Leone ${ }^{123 a, 123 b}$, K. Leonhardt ${ }^{44}$, C. Leonidopoulos ${ }^{46}$, S. Leontsinis ${ }^{10}$, C. Leroy ${ }^{94}$, C.G. Lester ${ }^{28}$, C.M. Lester ${ }^{121}$, M. Levchenko ${ }^{122}$, J. Levêque ${ }^{5}$, D. Levin ${ }^{88}$, L.J. Levinson ${ }^{173}$, M. Levy ${ }^{18}$, A. Lewis ${ }^{119}$, G.H. Lewis ${ }^{109}$, A.M. Leyko ${ }^{21}$, M. Leyton ${ }^{41}$, B. Li ${ }^{33 b, u}$, B. Li ${ }^{84}$, H. Li $^{149}$,

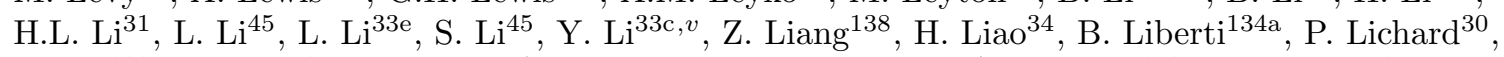
K. $\operatorname{Lie}^{166}$, J. Liebal ${ }^{21}$, W. Liebig ${ }^{14}$, C. Limbach ${ }^{21}$, A. Limosani ${ }^{87}$, S.C. $\operatorname{Lin}^{152, w}$, T.H. $\operatorname{Lin}^{82}$, 
F. Linde ${ }^{106}$, B.E. Lindquist ${ }^{149}$, J.T. Linnemann ${ }^{89}$, E. Lipeles ${ }^{121}$, A. Lipniacka ${ }^{14}$, M. Lisovyi ${ }^{42}$, T.M. $\operatorname{Liss}^{166}$, D. Lissauer ${ }^{25}$, A. Lister ${ }^{169}$, A.M. Litke ${ }^{138}$, B. Liu ${ }^{152}$, D. Liu ${ }^{152}$, J.B. Liu ${ }^{33 b}$, K. Liu ${ }^{33 b, x}$, L. Liu ${ }^{88}$, M. Liu ${ }^{45}$, M. Liu ${ }^{33 b}$, Y. Liu ${ }^{33 b}$, M. Livan ${ }^{120 a, 120 b}$, S.S.A. Livermore ${ }^{119}$, A. Lleres ${ }^{55}$, J. Llorente Merino ${ }^{81}$, S.L. Lloyd ${ }^{75}$, F. Lo Sterzo ${ }^{152}$, E. Lobodzinska ${ }^{42}$, P. Loch ${ }^{7}$, W.S. Lockman ${ }^{138}$, T. Loddenkoetter ${ }^{21}$, F.K. Loebinger ${ }^{83}$, A.E. Loevschall-Jensen ${ }^{36}$, A. $\operatorname{Loginov}^{177}$, T. Lohse ${ }^{16}$, K. Lohwasser ${ }^{42}$, M. Lokajicek ${ }^{126}$, V.P. Lombardo ${ }^{5}$, B.A. Long ${ }^{22}$, J.D. Long ${ }^{88}$, R.E. Long ${ }^{71}$, L. Lopes ${ }^{125 a}$, D. Lopez Mateos ${ }^{57}$, B. Lopez Paredes ${ }^{140}$, I. Lopez Paz ${ }^{12}$, J. Lorenz ${ }^{99}$, N. Lorenzo Martinez ${ }^{60}$, M. Losada ${ }^{163}$, P. Loscutoff ${ }^{15}$, X. Lou ${ }^{41}$, A. Lounis ${ }^{116}$,

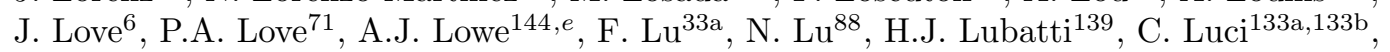

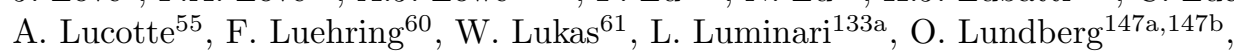
B. Lund-Jensen ${ }^{148}$, M. Lungwitz ${ }^{82}$, D. Lynn $^{25}$, R. Lysak ${ }^{126}$, E. Lytken ${ }^{80}$, H. Ma ${ }^{25}$, L.L. Ma ${ }^{33 d}$, G. Maccarrone ${ }^{47}$, A. Macchiolo ${ }^{100}$, J. Machado Miguens ${ }^{125 a, 125 b}$, D. Macina ${ }^{30}$, D. Madaffari ${ }^{84}$, R. Madar $^{48}$, H.J. Maddocks ${ }^{71}$, W.F. Mader ${ }^{44}$, A. Madsen ${ }^{167}$, M. Maeno ${ }^{8}$, T. Maeno ${ }^{25}$,

E. Magradze ${ }^{54}$, K. Mahboubi ${ }^{48}$, J. Mahlstedt ${ }^{106}$, S. Mahmoud $^{73}$, C. Maiani $^{137}$,

C. Maidantchik ${ }^{24 a}$, A.A. Maier ${ }^{100}$, A. Maio ${ }^{125 a, 125 b, 125 d}$, S. Majewski ${ }^{115}$, Y. Makida ${ }^{65}$, N. Makovec ${ }^{116}$, P. Mal ${ }^{137, y}$, B. Malaescu ${ }^{79}$, Pa. Malecki ${ }^{39}$, V.P. Maleev ${ }^{122}$, F. Malek ${ }^{55}$, U. Mallik ${ }^{62}$, D. Malon ${ }^{6}$, C. Malone ${ }^{144}$, S. Maltezos ${ }^{10}$, V.M. Malyshev ${ }^{108}$, S. Malyukov ${ }^{30}$, J. Mamuzic ${ }^{13 b}$, B. Mandelli ${ }^{30}$, L. Mandelli ${ }^{90 a}$, I. Mandić ${ }^{74}$, R. Mandrysch ${ }^{62}$, J. Maneira ${ }^{125 a, 125 b}$, A. Manfredini ${ }^{100}$, L. Manhaes de Andrade Filho ${ }^{24 b}$, J.A. Manjarres Ramos ${ }^{160 b}$, A. Mann ${ }^{99}$, P.M. Manning ${ }^{138}$, A. Manousakis-Katsikakis ${ }^{9}$, B. Mansoulie ${ }^{137}$, R. Mantifel ${ }^{86}$, L. Mapelli ${ }^{30}$, L. March $^{168}$, J.F. Marchand ${ }^{29}$, G. Marchiori ${ }^{79}$, M. Marcisovsky ${ }^{126}$, C.P. Marino ${ }^{170}$, M. Marjanovic ${ }^{13 a}$, C.N. Marques ${ }^{125 a}$, F. Marroquim ${ }^{24 a}$, S.P. Marsden ${ }^{83}$, Z. Marshall ${ }^{15}$,

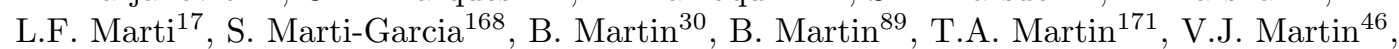
B. Martin dit Latour ${ }^{14}$, H. Martinez ${ }^{137}$, M. Martinez ${ }^{12, n}$, S. Martin-Haugh ${ }^{130}$, A.C. Martyniuk ${ }^{77}$,

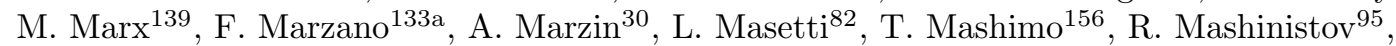
J. Masik ${ }^{83}$, A.L. Maslennikov ${ }^{108}$, I. Massa ${ }^{20 a, 20 b}$, L. Massa ${ }^{20 a, 20 b}$, N. Massol ${ }^{5}$, P. Mastrandrea ${ }^{149}$, A. Mastroberardino $37 \mathrm{a}, 37 \mathrm{~b}$, T. Masubuchi ${ }^{156}$, P. Mättig ${ }^{176}$, J. Mattmann ${ }^{82}$, J. Maurer ${ }^{26 a}$, S.J. Maxfield ${ }^{73}$, D.A. Maximov ${ }^{108, t}$, R. Mazini ${ }^{152}$, L. Mazzaferro ${ }^{134 a, 134 b}$, G. Mc Goldrick ${ }^{159}$, S.P. Mc Kee ${ }^{88}$, A. McCarn ${ }^{88}$, R.L. McCarthy ${ }^{149}$, T.G. McCarthy ${ }^{29}$, N.A. McCubbin ${ }^{130}$, K.W. McFarlane ${ }^{56, *}$, J.A. Mcfayden ${ }^{77}$, G. Mchedlidze ${ }^{54}$, S.J. McMahon ${ }^{130}$, R.A. McPherson ${ }^{170, i}$, A. Meade ${ }^{85}$, J. Mechnich ${ }^{106}$, M. Medinnis ${ }^{42}$, S. Meehan ${ }^{31}$, S. Mehlhase ${ }^{99}$, A. Mehta ${ }^{73}$,

K. Meier ${ }^{58 a}$, C. Meineck ${ }^{99}$, B. Meirose ${ }^{80}$, C. Melachrinos ${ }^{31}$, B.R. Mellado Garcia ${ }^{146 c}$, F. Meloni ${ }^{17}$, A. Mengarelli $20 \mathrm{a}, 20 \mathrm{~b}, \mathrm{~S} . \mathrm{Menke}^{100}$, E. Meoni ${ }^{162}$, K.M. Mercurio ${ }^{57}$, S. Mergelmeyer ${ }^{21}$, N. Meric ${ }^{137}$, P. Mermod ${ }^{49}$, L. Merola 103a,103b, C. Meroni ${ }^{90 a}$, F.S. Merritt ${ }^{31}$, H. Merritt ${ }^{110}$, A. Messina ${ }^{30, z}$, J. Metcalfe ${ }^{25}$, A.S. Mete ${ }^{164}$, C. Meyer ${ }^{82}$, C. Meyer ${ }^{121}$, J-P. Meyer ${ }^{137}$, J. Meyer ${ }^{30}$, R.P. Middleton ${ }^{130}$, S. Migas ${ }^{73}$, L. Mijović ${ }^{21}$, G. Mikenberg ${ }^{173}$, M. Mikestikova ${ }^{126}$, M. Mikuž ${ }^{74}$, A. Milic ${ }^{30}$, D.W. Miller ${ }^{31}$, C. Mills ${ }^{46}$, A. Milov ${ }^{173}$, D.A. Milstead ${ }^{147 a, 147 b}$, D. Milstein ${ }^{173}$,

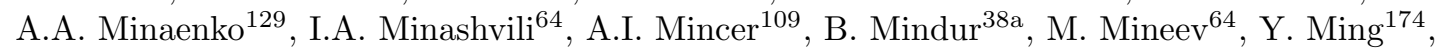
L.M. Mir ${ }^{12}$, G. Mirabelli133a, T. Mitani ${ }^{172}$, J. Mitrevski ${ }^{99}$, V.A. Mitsou ${ }^{168}$, S. Mitsui ${ }^{65}$, A. Miucci ${ }^{49}$, P.S. Miyagawa ${ }^{140}$, J.U. Mjörnmark ${ }^{80}$, T. Moa ${ }^{147 a, 147 b}$, K. Mochizuki ${ }^{84}$, S. Mohapatra ${ }^{35}$, W. Mohr ${ }^{48}$, S. Molander ${ }^{147 a, 147 b}$, R. Moles-Valls ${ }^{168}$, K. Mönig ${ }^{42}$, C. Monini ${ }^{55}$, J. Monk ${ }^{36}$, E. Monnier ${ }^{84}$, J. Montejo Berlingen ${ }^{12}$, F. Monticelli ${ }^{70}$, S. Monzani ${ }^{133 a, 133 b}$, R.W. Moore ${ }^{3}$, A. Moraes ${ }^{53}$, N. Morange ${ }^{62}$, D. Moreno ${ }^{82}$, M. Moreno Llácer ${ }^{54}$, P. Morettini ${ }^{50 a}$, M. Morgenstern ${ }^{44}$, M. Morii ${ }^{57}$, S. Moritz ${ }^{82}$, A.K. Morley ${ }^{148}$, G. Mornacchi ${ }^{30}$, J.D. Morris ${ }^{75}$, L. Morvaj ${ }^{102}$, H.G. Moser ${ }^{100}$, M. Mosidze ${ }^{51 b}$, J. Moss ${ }^{110}$, K. Motohashi ${ }^{158}$, R. Mount ${ }^{144}$, E. Mountricha ${ }^{25}$, S.V. Mouraviev ${ }^{95, *}$, E.J.W. Moyse ${ }^{85}$, S. Muanza ${ }^{84}$, R.D. Mudd ${ }^{18}$, F. Mueller ${ }^{58 a}$, J. Mueller ${ }^{124}$, K. Mueller ${ }^{21}$, T. Mueller ${ }^{28}$, T. Mueller ${ }^{82}$, D. Muenstermann ${ }^{49}$, Y. Munwes ${ }^{154}$, J.A. Murillo Quijada ${ }^{18}$, W.J. Murray ${ }^{171,130}$, H. Musheghyan ${ }^{54}$, E. Musto ${ }^{153}$, A.G. Myagkov ${ }^{129, a a}$,

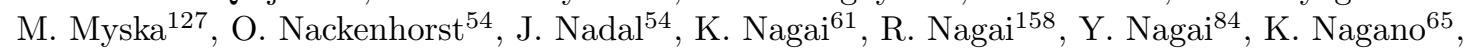

A. Nagarkar ${ }^{110}$, Y. Nagasaka ${ }^{59}$, M. Nagel ${ }^{100}$, A.M. Nairz ${ }^{30}$, Y. Nakahama ${ }^{30}$, K. Nakamura ${ }^{65}$,

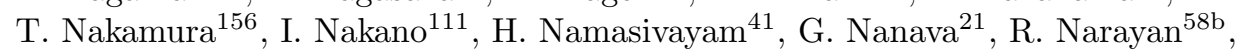

T. Nattermann ${ }^{21}$, T. Naumann ${ }^{42}$, G. Navarro ${ }^{163}$, R. Nayyar ${ }^{7}$, H.A. Neal ${ }^{88}$, P.Yu. Nechaeva ${ }^{95}$, 
T.J. Neep ${ }^{83}$, P.D. Nef ${ }^{144}$, A. Negri ${ }^{120 a, 120 b}$, G. Negri ${ }^{30}$, M. Negrini ${ }^{20 a}$, S. Nektarijevic ${ }^{49}$, A. Nelson ${ }^{164}$, T.K. Nelson ${ }^{144}$, S. Nemecek ${ }^{126}$, P. Nemethy ${ }^{109}$, A.A. Nepomuceno ${ }^{24 a}$, M. Nessi $^{30, a b}$, M.S. Neubauer ${ }^{166}$, M. Neumann ${ }^{176}$, R.M. Neves ${ }^{109}$, P. Nevski ${ }^{25}$, P.R. Newman ${ }^{18}$, D.H. Nguyen ${ }^{6}$, R.B. Nickerson ${ }^{119}$, R. Nicolaidou ${ }^{137}$, B. Nicquevert ${ }^{30}$, J. Nielsen ${ }^{138}$, N. Nikiforou ${ }^{35}$, A. Nikiforov ${ }^{16}$, V. Nikolaenko ${ }^{129, a a}$, I. Nikolic-Audit ${ }^{79}$, K. Nikolics $^{49}$, K. Nikolopoulos ${ }^{18}$, P. Nilsson ${ }^{8}$, Y. Ninomiya ${ }^{156}$, A. Nisati ${ }^{133 a}$, R. Nisius ${ }^{100}$, T. Nobe ${ }^{158}$, L. Nodulman $^{6}$, M. Nomachi ${ }^{117}$, I. Nomidis ${ }^{29}$, S. Norberg ${ }^{112}$, M. Nordberg ${ }^{30}$, O. Novgorodova ${ }^{44}$, S. Nowak ${ }^{100}$, M. Nozaki ${ }^{65}$, L. Nozka ${ }^{114}$, K. Ntekas ${ }^{10}$, G. Nunes Hanninger ${ }^{87}$, T. Nunnemann ${ }^{99}$, E. Nurse ${ }^{77}$, F. Nuti ${ }^{87}$, B.J. O’Brien ${ }^{46}$, F. O'grady ${ }^{7}$, D.C. O’Neil ${ }^{143}$, V. O'Shea ${ }^{53}$, F.G. Oakham ${ }^{29, d}$, H. Oberlack ${ }^{100}$, T. Obermann ${ }^{21}$, J. Ocariz ${ }^{79}$, A. Ochi ${ }^{66}$, M.I. Ochoa ${ }^{77}$, S. Oda ${ }^{69}$, S. Odaka ${ }^{65}$, H. Ogren ${ }^{60}$, A. $\mathrm{Oh}^{83}$, S.H. $\mathrm{Oh}^{45}$, C.C. Ohm ${ }^{15}$, H. Ohman ${ }^{167}$, W. Okamura ${ }^{117}$, H. Okawa ${ }^{25}$, Y. Okumura ${ }^{31}$, T. Okuyama ${ }^{156}$, A. Olariu ${ }^{26 a}$, A.G. Olchevski ${ }^{64}$, S.A. Olivares Pino ${ }^{46}$, D. Oliveira Damazio ${ }^{25}$, E. Oliver Garcia ${ }^{168}$, A. Olszewski ${ }^{39}$, J. Olszowska ${ }^{39}$, A. Onofre ${ }^{125 a, 125 e}$, P.U.E. Onyisi ${ }^{31, o}$, C.J. Oram ${ }^{160 a}$, M.J. Oreglia ${ }^{31}$, Y. Oren ${ }^{154}$, D. Orestano ${ }^{135 a, 135 b}$,

N. Orlando ${ }^{72 a}, 72 b$, C. Oropeza Barrera ${ }^{53}$, R.S. Orr ${ }^{159}$, B. Osculati ${ }^{50 a, 50 b}$, R. Ospanov ${ }^{121}$, G. Otero y Garzon ${ }^{27}$, H. Otono ${ }^{69}$, M. Ouchrif ${ }^{136 d}$, E.A. Ouellette ${ }^{170}$, F. Ould-Saada ${ }^{118}$, A. Ouraou ${ }^{137}$, K.P. Oussoren ${ }^{106}$, Q. Ouyang ${ }^{33 a}$, A. Ovcharova ${ }^{15}$, M. Owen ${ }^{83}$, V.E. Ozcan ${ }^{19 a}$, N. Ozturk ${ }^{8}$, K. Pachal ${ }^{119}$, A. Pacheco Pages ${ }^{12}$, C. Padilla Aranda ${ }^{12}$, M. Pagáčováa ${ }^{48}$,

S. Pagan Griso ${ }^{15}$, E. Paganis ${ }^{140}$, C. Pahl ${ }^{100}$, F. Paige ${ }^{25}$, P. Pais ${ }^{85}$, K. Pajchel ${ }^{118}$, G. Palacino ${ }^{160 b}$, S. Palestini ${ }^{30}$, M. Palka ${ }^{38 b}$, D. Pallin ${ }^{34}$, A. Palma ${ }^{125 a, 125 b}$, J.D. Palmer ${ }^{18}$, Y.B. Pan ${ }^{174}$,

E. Panagiotopoulou ${ }^{10}$, J.G. Panduro Vazquez ${ }^{76}$, P. Pani ${ }^{106}$, N. Panikashvili ${ }^{88}$, S. Panitkin ${ }^{25}$,

D. Pantea ${ }^{26 a}$, L. Paolozzi ${ }^{134 a, 134 b}$, Th.D. Papadopoulou ${ }^{10}$, K. Papageorgiou ${ }^{155, l}$, A. Paramonov ${ }^{6}$, D. Paredes Hernandez ${ }^{34}$, M.A. Parker ${ }^{28}$, F. Parodi ${ }^{50 a, 50 b}$, J.A. Parsons ${ }^{35}$, U. Parzefall ${ }^{48}$, E. Pasqualucci ${ }^{133 a}$, S. Passaggio ${ }^{50 a}$, A. Passeri ${ }^{135 a}$, F. Pastore ${ }^{135 a, 135 b, *}$, Fr. Pastore ${ }^{76}$, G. Pásztor ${ }^{29}$, S. Pataraia ${ }^{176}$, N.D. Patel ${ }^{151}$, J.R. Pater ${ }^{83}$, S. Patricelli103a,103b, T. Pauly ${ }^{30}$, J. Pearce ${ }^{170}$, M. Pedersen ${ }^{118}$, S. Pedraza Lopez ${ }^{168}$, R. Pedro ${ }^{125 a, 125 b}$, S.V. Peleganchuk ${ }^{108}$, D. Pelikan ${ }^{167}$, H. Peng ${ }^{33 b}$, B. Penning ${ }^{31}$, J. Penwell ${ }^{60}$, D.V. Perepelitsa ${ }^{25}$, E. Perez Codina ${ }^{160 a}$, M.T. Pérez García-Estañ ${ }^{168}$, V. Perez Reale ${ }^{35}$, L. Perini ${ }^{90 a, 90 b}$, H. Pernegger $^{30}$, R. Perrino ${ }^{72 a}$, R. Peschke ${ }^{42}$, V.D. Peshekhonov ${ }^{64}$, K. Peters $^{30}$, R.F.Y. Peters ${ }^{83}$, B.A. Petersen ${ }^{30}$, T.C. Petersen ${ }^{36}$, E. Petit ${ }^{42}$, A. Petridis ${ }^{147 a, 147 b}$, C. Petridou ${ }^{155}$, E. Petrolo ${ }^{133 a}$,

F. Petrucci ${ }^{135 a, 135 b}$, N.E. Pettersson ${ }^{158}$, R. Pezoa ${ }^{32 b}$, P.W. Phillips ${ }^{130}$, G. Piacquadio ${ }^{144}$, E. Pianori ${ }^{171}$, A. Picazio ${ }^{49}$, E. Piccaro ${ }^{75}$, M. Piccinini ${ }^{20 a, 20 b}$, R. Piegaia ${ }^{27}$, D.T. Pignotti ${ }^{110}$, J.E. Pilcher ${ }^{31}$, A.D. Pilkington ${ }^{77}$, J. Pina125a,125b,125d, M. Pinamonti165a,165c,ac , A. Pinder ${ }^{119}$, J.L. Pinfold ${ }^{3}$, A. Pingel ${ }^{36}$, B. Pinto ${ }^{125 a}$, S. Pires ${ }^{79}$, M. Pitt ${ }^{173}$, C. Pizio ${ }^{90 a, 90 b}$, L. Plazak ${ }^{145 a}$, M.-A. Pleier ${ }^{25}$, V. Pleskot ${ }^{128}$, E. Plotnikova ${ }^{64}$, P. Plucinski147a,147b, S. Poddar ${ }^{58 a}$, F. Podlyski ${ }^{34}$, R. Poettgen ${ }^{82}$, L. Poggioli ${ }^{116}$, D. Pohl ${ }^{21}$, M. Pohl ${ }^{49}$, G. Polesello ${ }^{120 a}$, A. Policicchio ${ }^{37 a, 37 b}$, R. Polifka ${ }^{159}$, A. Polini ${ }^{20 a}$, C.S. Pollard ${ }^{45}$, V. Polychronakos ${ }^{25}$, K. Pommès ${ }^{30}$, L. Pontecorvo ${ }^{133 a}$, B.G. Pope ${ }^{89}$, G.A. Popeneciu ${ }^{26 b}$, D.S. Popovic ${ }^{13 a}$, A. Poppleton ${ }^{30}$, X. Portell Bueso ${ }^{12}$, S. Pospisil ${ }^{127}$, K. Potamianos ${ }^{15}$, I.N. Potrap ${ }^{64}$, C.J. Potter ${ }^{150}$, C.T. Potter ${ }^{115}$, G. Poulard ${ }^{30}$, J. Poveda ${ }^{60}$, V. Pozdnyakov ${ }^{64}$, P. Pralavorio ${ }^{84}$, A. Pranko ${ }^{15}$, S. Prasad ${ }^{30}$, R. Pravahan ${ }^{8}$, S. Prell ${ }^{63}$, D. Price ${ }^{83}$, J. Price ${ }^{73}$, L.E. Price ${ }^{6}$, D. Prieur ${ }^{124}$, M. Primavera ${ }^{72 a}$, M. Proissl ${ }^{46}$, K. Prokofiev ${ }^{47}$, F. Prokoshin ${ }^{32 b}$, E. Protopapadaki ${ }^{137}$, S. Protopopescu ${ }^{25}$, J. Proudfoot ${ }^{6}$, M. Przybycien ${ }^{38 a}$, H. Przysiezniak ${ }^{5}$, E. Ptacek ${ }^{115}$, D. Puddu ${ }^{135 a, 135 b}$, E. Pueschel ${ }^{85}$, D. Puldon ${ }^{149}$,

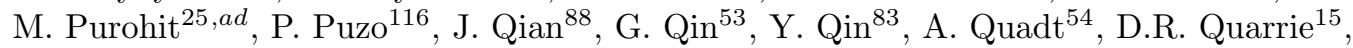
W.B. Quayle ${ }^{165 a, 165 b}$, M. Queitsch-Maitland ${ }^{83}$, D. Quilty ${ }^{53}$, A. Qureshi ${ }^{160 b}$, V. Radeka ${ }^{25}$, V. Radescu ${ }^{42}$, S.K. Radhakrishnan ${ }^{149}$, P. Radloff ${ }^{115}$, P. Rados ${ }^{87}$, F. Ragusa ${ }^{90 a, 90 b}$, G. Rahal ${ }^{179}$, S. Rajagopalan ${ }^{25}$, M. Rammensee ${ }^{30}$, A.S. Randle-Conde ${ }^{40}$, C. Rangel-Smith ${ }^{167}$, K. Rao ${ }^{164}$, F. Rauscher ${ }^{99}$, T.C. Rave ${ }^{48}$, T. Ravenscroft ${ }^{53}$, M. Raymond ${ }^{30}$, A.L. $\operatorname{Read}^{118}$, N.P. Readioff ${ }^{73}$, D.M. Rebuzzi ${ }^{120 a, 120 b}$, A. Redelbach ${ }^{175}$, G. Redlinger ${ }^{25}$, R. Reece ${ }^{138}$, K. Reeves ${ }^{41}$, L. Rehnisch ${ }^{16}$, H. Reisin ${ }^{27}$, M. Relich ${ }^{164}$, C. Rembser ${ }^{30}$, H. Ren ${ }^{33 a}$, Z.L. Ren ${ }^{152}$, A. Renaud ${ }^{116}$, M. Rescigno ${ }^{133 a}$, S. Resconi ${ }^{90 a}$, O.L. Rezanova ${ }^{108, t}$, P. Reznicek ${ }^{128}$, R. Rezvani ${ }^{94}$, R. Richter $^{100}$, M. Ridel ${ }^{79}$, P. Rieck ${ }^{16}$, J. Rieger ${ }^{54}$, M. Rijssenbeek ${ }^{149}$, A. Rimoldi ${ }^{120 a, 120 b}$, L. Rinaldi ${ }^{20 a}$, E. Ritsch $^{61}$, I. Riu ${ }^{12}$, 
F. Rizatdinova ${ }^{113}$, E. Rizvi ${ }^{75}$, S.H. Robertson ${ }^{86, i}$, A. Robichaud-Veronneau ${ }^{86}$, D. Robinson ${ }^{28}$, J.E.M. Robinson ${ }^{83}$, A. Robson ${ }^{53}$, C. Roda ${ }^{123 a, 123 b}$, L. Rodrigues ${ }^{30}$, S. Roe ${ }^{30}$, O. Røhne ${ }^{118}$, S. Rolli ${ }^{162}$, A. Romaniouk ${ }^{97}$, M. Romano ${ }^{20 a, 20 b}$, E. Romero Adam ${ }^{168}$, N. Rompotis ${ }^{139}$, M. Ronzani ${ }^{48}$, L. Roos ${ }^{79}$, E. $\operatorname{Ros}^{168}$, S. Rosati ${ }^{133 a}$, K. Rosbach ${ }^{49}$, M. Rose $^{76}$, P. Rose ${ }^{138}$, P.L. Rosendahl ${ }^{14}$, O. Rosenthal ${ }^{142}$, V. Rossetti147a,147b, E. Rossi ${ }^{103 a, 103 b}$, L.P. Rossi ${ }^{50 a}$, R. Rosten ${ }^{139}$, M. Rotaru ${ }^{26 a}$, I. Roth ${ }^{173}$, J. Rothberg ${ }^{139}$, D. Rousseau ${ }^{116}$, C.R. Royon ${ }^{137}$, A. Rozanov ${ }^{84}$, Y. Rozen ${ }^{153}$, X. Ruan ${ }^{146 c}$, F. Rubbo ${ }^{12}$, I. Rubinskiy ${ }^{42}$, V.I. Rud ${ }^{98}$, C. Rudolph $^{44}$, M.S. Rudolph ${ }^{159}$, F. Rühr ${ }^{48}$, A. Ruiz-Martinez ${ }^{30}$, Z. Rurikova ${ }^{48}$, N.A. Rusakovich ${ }^{64}$,

A. Ruschke ${ }^{99}$, J.P. Rutherfoord ${ }^{7}$, N. Ruthmann ${ }^{48}$, Y.F. Ryabov ${ }^{122}$, M. Rybar ${ }^{128}$, G. Rybkin ${ }^{116}$, N.C. Ryder ${ }^{119}$, A.F. Saavedra ${ }^{151}$, S. Sacerdoti ${ }^{27}$, A. Saddique ${ }^{3}$, I. Sadeh ${ }^{154}$,

H.F-W. Sadrozinski ${ }^{138}$, R. Sadykov ${ }^{64}$, F. Safai Tehrani ${ }^{133 a}$, H. Sakamoto ${ }^{156}$, Y. Sakurai ${ }^{172}$, G. Salamanna ${ }^{135 a, 135 b}$, A. Salamon ${ }^{134 a}$, M. Saleem ${ }^{112}$, D. Salek ${ }^{106}$, P.H. Sales De Bruin ${ }^{139}$, D. Salihagic ${ }^{100}$, A. Salnikov ${ }^{144}$, J. Salt ${ }^{168}$, D. Salvatore ${ }^{37 a, 37 b}$, F. Salvatore ${ }^{150}$, A. Salvucci ${ }^{105}$, A. Salzburger ${ }^{30}$, D. Sampsonidis ${ }^{155}$, A. Sanchez ${ }^{103 a, 103 b}$, J. Sánchez ${ }^{168}$, V. Sanchez Martinez ${ }^{168}$, H. Sandaker ${ }^{14}$, R.L. Sandbach ${ }^{75}$, H.G. Sander ${ }^{82}$, M.P. Sanders ${ }^{99}$, M. Sandhoff ${ }^{176}$, T. Sandoval $^{28}$, C. Sandoval ${ }^{163}$, R. Sandstroem ${ }^{100}$, D.P.C. Sankey ${ }^{130}$, A. Sansoni ${ }^{47}$, C. Santoni ${ }^{34}$, R. Santonico ${ }^{134 a, 134 b}$, H. Santos ${ }^{125 a}$, I. Santoyo Castillo ${ }^{150}$, K. Sapp ${ }^{124}$, A. Sapronov ${ }^{64}$, J.G. Saraiva ${ }^{125 a, 125 d}$, B. Sarrazin ${ }^{21}$, G. Sartisohn ${ }^{176}$, O. Sasaki ${ }^{65}$, Y. Sasaki ${ }^{156}$, G. Sauvage ${ }^{5, *}$, E. Sauvan $^{5}$, P. Savard ${ }^{159, d}$, D.O. Savu ${ }^{30}$, C. Sawyer ${ }^{119}$, L. Sawyer ${ }^{78, m}$, D.H. Saxon ${ }^{53}$, J. Saxon ${ }^{121}$, C. Sbarra ${ }^{20 a}$, A. Sbrizzi ${ }^{3}$, T. Scanlon ${ }^{77}$, D.A. Scannicchio ${ }^{164}$, M. Scarcella ${ }^{151}$, V. Scarfone ${ }^{37 a, 37 b}$, J. Schaarschmidt ${ }^{173}$, P. Schacht ${ }^{100}$, D. Schaefer ${ }^{30}$, R. Schaefer ${ }^{42}$, S. Schaepe ${ }^{21}$, S. Schaetzel ${ }^{58 b}$, U. Schäfer ${ }^{82}$, A.C. Schaffer ${ }^{116}$, D. Schaile ${ }^{99}$, R.D. Schamberger ${ }^{149}$, V. Scharf ${ }^{58 a}$,

V.A. Schegelsky ${ }^{122}$, D. Scheirich ${ }^{128}$, M. Schernau ${ }^{164}$, M.I. Scherzer ${ }^{35}$, C. Schiavi ${ }^{50 a, 50 b}$ J. Schieck ${ }^{99}$, C. Schillo ${ }^{48}$, M. Schioppa ${ }^{37 a, 37 b}$, S. Schlenker ${ }^{30}$, E. Schmidt ${ }^{48}$, K. Schmieden ${ }^{30}$, C. Schmitt $^{82}$, C. Schmitt ${ }^{99}$, S. Schmitt ${ }^{58 b}$, B. Schneider ${ }^{17}$, Y.J. Schnellbach ${ }^{73}$, U. Schnoor ${ }^{44}$, L. Schoeffel ${ }^{137}$, A. Schoening ${ }^{58 b}$, B.D. Schoenrock ${ }^{89}$, A.L.S. Schorlemmer ${ }^{54}$, M. Schott ${ }^{82}$, D. Schouten ${ }^{160 a}$, J. Schovancova ${ }^{25}$, S. Schramm ${ }^{159}$, M. Schreyer ${ }^{175}$, C. Schroeder ${ }^{82}$, N. Schuh ${ }^{82}$, M.J. Schultens ${ }^{21}$, H.-C. Schultz-Coulon ${ }^{58 a}$, H. Schulz ${ }^{16}$, M. Schumacher ${ }^{48}$, B.A. Schumm ${ }^{138}$, Ph. Schune ${ }^{137}$, C. Schwanenberger ${ }^{83}$, A. Schwartzman ${ }^{144}$, Ph. Schwegler ${ }^{100}$, Ph. Schwemling ${ }^{137}$, R. Schwienhorst ${ }^{89}$, J. Schwindling ${ }^{137}$, T. Schwindt ${ }^{21}$, M. Schwoerer ${ }^{5}$, F.G. Sciacca ${ }^{17}$, E. Scifo ${ }^{116}$, G. Sciolla ${ }^{23}$, W.G. Scott ${ }^{130}$, F. Scuri ${ }^{123 a, 123 b}$, F. Scutti ${ }^{21}$, J. Searcy ${ }^{88}$, G. Sedov ${ }^{42}$, E. Sedykh ${ }^{122}$, S.C. Seidel ${ }^{104}$, A. Seiden ${ }^{138}$, F. Seifert ${ }^{127}$, J.M. Seixas ${ }^{24 a}$, G. Sekhniaidze ${ }^{103 a}$, S.J. Sekula ${ }^{40}$, K.E. Selbach ${ }^{46}$, D.M. Seliverstov ${ }^{122, *}$, G. Sellers ${ }^{73}$, N. Semprini-Cesari ${ }^{20 a, 20 b}$, C. Serfon ${ }^{30}$, L. $\operatorname{Serin}^{116}$, L. Serkin ${ }^{54}$, T. Serre ${ }^{84}$, R. Seuster ${ }^{160 a}$, H. Severini ${ }^{112}$, T. Sfiligoj74, F. Sforza ${ }^{100}$, A. Sfyrla ${ }^{30}$, E. Shabalina ${ }^{54}$, M. Shamim ${ }^{115}$, L.Y. Shan ${ }^{33 a}$, R. Shang ${ }^{166}$, J.T. Shank ${ }^{22}$,

M. Shapiro ${ }^{15}$, P.B. Shatalov ${ }^{96}$, K. Shaw ${ }^{165 a, 165 b}$, C.Y. Shehu ${ }^{150}$, P. Sherwood ${ }^{77}$, L. Shi ${ }^{152, a e}$, S. Shimizu ${ }^{66}$, C.O. Shimmin ${ }^{164}$, M. Shimojima ${ }^{101}$, M. Shiyakova ${ }^{64}$, A. Shmeleva ${ }^{95}$, M.J. Shochet ${ }^{31}$, D. Short ${ }^{119}$, S. Shrestha ${ }^{63}$, E. Shulga ${ }^{97}$, M.A. Shupe ${ }^{7}$, S. Shushkevich ${ }^{42}$, P. Sicho ${ }^{126}$, O. Sidiropoulou ${ }^{155}$, D. Sidorov ${ }^{113}$, A. Sidoti133a, F. Siegert ${ }^{44}$, Dj. Sijacki ${ }^{13 a}$, J. Silva ${ }^{125 a, 125 d}$, Y. Silver ${ }^{154}$, D. Silverstein ${ }^{144}$, S.B. Silverstein ${ }^{147 a}$, V. Simak ${ }^{127}$, O. Simard ${ }^{5}$, Lj. Simic ${ }^{13 a}$, S. Simion ${ }^{116}$, E. Simioni ${ }^{82}$, B. Simmons ${ }^{77}$, R. Simoniello90a,90b, M. Simonyan ${ }^{36}$, P. Sinervo ${ }^{159}$, N.B. Sinev ${ }^{115}$, V. Sipica ${ }^{142}$, G. Siragusa ${ }^{175}$, A. Sircar ${ }^{78}$, A.N. Sisakyan ${ }^{64, *}$, S.Yu. Sivoklokov ${ }^{98}$, J. Sjölin ${ }^{147 a, 147 b}$, T.B. Sjursen ${ }^{14}$, H.P. Skottowe ${ }^{57}$, K.Yu. Skovpen ${ }^{108}$, P. Skubic ${ }^{112}$, M. Slater ${ }^{18}$, T. Slavicek ${ }^{127}$, K. Sliwa ${ }^{162}$, V. Smakhtin ${ }^{173}$, B.H. Smart ${ }^{46}$,

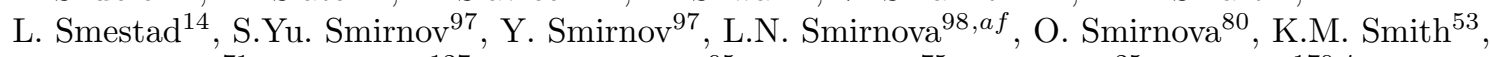
M. Smizanska ${ }^{71}$, K. Smolek ${ }^{127}$, A.A. Snesarev ${ }^{95}$, G. Snidero ${ }^{75}$, S. Snyder ${ }^{25}$, R. Sobie ${ }^{170, i}$, F. Socher ${ }^{44}$, A. Soffer ${ }^{154}$, D.A. Soh ${ }^{152, a e}$, C.A. Solans ${ }^{30}$, M. Solar ${ }^{127}$, J. Solc ${ }^{127}$, E.Yu. Soldatov ${ }^{97}$, U. Soldevila ${ }^{168}$, A.A. Solodkov ${ }^{129}$, A. Soloshenko ${ }^{64}$, O.V. Solovyanov ${ }^{129}$, V. Solovyev ${ }^{122}$, P. Sommer ${ }^{48}$, H.Y. Song ${ }^{33 b}$, N. Soni ${ }^{1}$, A. Sood ${ }^{15}$, A. Sopczak ${ }^{127}$, B. Sopko ${ }^{127}$, V. Sopko ${ }^{127}$, V. Sorin ${ }^{12}$, M. Sosebee ${ }^{8}$, R. Soualah ${ }^{165 a, 165 c}$, P. Soueid ${ }^{94}$, A.M. Soukharev ${ }^{108}$, D. South ${ }^{42}$,

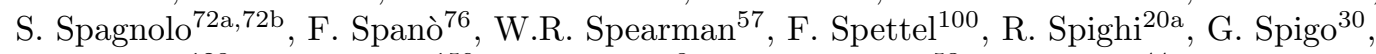

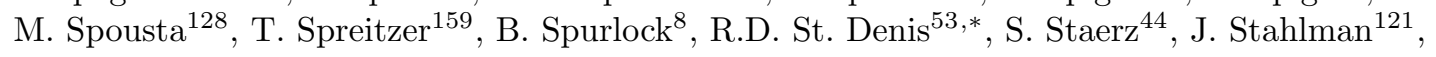


R. Stamen ${ }^{58 a}$, E. Stanecka ${ }^{39}$, R.W. Stanek ${ }^{6}$, C. Stanescu ${ }^{135 a}$, M. Stanescu-Bellu ${ }^{42}$, M.M. Stanitzki ${ }^{42}$, S. Stapnes ${ }^{118}$, E.A. Starchenko ${ }^{129}$, J. Stark ${ }^{55}$, P. Staroba ${ }^{126}$, P. Starovoitov ${ }^{42}$, R. Staszewski ${ }^{39}$, P. Stavina ${ }^{145 a, *}$, P. Steinberg ${ }^{25}$, B. Stelzer ${ }^{143}$, H.J. Stelzer ${ }^{30}$,

O. Stelzer-Chilton ${ }^{160 a}$, H. Stenzel ${ }^{52}$, S. Stern ${ }^{100}$, G.A. Stewart ${ }^{53}$, J.A. Stillings ${ }^{21}$, M.C. Stockton ${ }^{86}$, M. Stoebe ${ }^{86}$, G. Stoicea ${ }^{26 a}$, P. Stolte ${ }^{54}$, S. Stonjek ${ }^{100}$, A.R. Stradling ${ }^{8}$, A. Straessner ${ }^{44}$,

M.E. Stramaglia ${ }^{17}$, J. Strandberg ${ }^{148}$, S. Strandberg ${ }^{147 a, 147 b}$, A. Strandlie ${ }^{118}$, E. Strauss ${ }^{144}$, M. Strauss ${ }^{112}$, P. Strizenec ${ }^{145 b}$, R. Ströhmer ${ }^{175}$, D.M. Strom ${ }^{115}$, R. Stroynowski ${ }^{40}$, S.A. Stucci ${ }^{17}$, B. Stugu ${ }^{14}$, N.A. Styles ${ }^{42}$, D. $\mathrm{Su}^{144}$, J. Su ${ }^{124}$, R. Subramaniam ${ }^{78}$, A. Succurro ${ }^{12}$, Y. Sugaya ${ }^{117}$, C. Suhr ${ }^{107}$, M. Suk ${ }^{127}$, V.V. Sulin ${ }^{95}$, S. Sultansoy ${ }^{4 c}$, T. Sumida ${ }^{67}$, S. Sun ${ }^{57}$, X. Sun ${ }^{33 a}$, J.E. Sundermann ${ }^{48}$, K. Suruliz ${ }^{140}$, G. Susinno ${ }^{37 a, 37 b}$, M.R. Sutton ${ }^{150}$, Y. Suzuki ${ }^{65}$, M. Svatos ${ }^{126}$, S. Swedish ${ }^{169}$, M. Swiatlowski ${ }^{144}$, I. Sykora ${ }^{145 a}$, T. Sykora ${ }^{128}$, D. Ta ${ }^{89}$, C. Taccini ${ }^{135 a, 135 b}$,

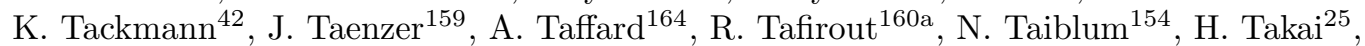
R. Takashima ${ }^{68}$, H. Takeda ${ }^{66}$, T. Takeshita ${ }^{141}$, Y. Takubo ${ }^{65}$, M. Talby ${ }^{84}$, A.A. Talyshev ${ }^{108, t}$, J.Y.C. Tam ${ }^{175}$, K.G. Tan ${ }^{87}$, J. Tanaka ${ }^{156}$, R. Tanaka ${ }^{116}$, S. Tanaka ${ }^{132}$, S. Tanaka ${ }^{65}$, A.J. Tanasijczuk ${ }^{143}$, B.B. Tannenwald ${ }^{110}$, N. Tannoury ${ }^{21}$, S. Tapprogge ${ }^{82}$, S. Tarem ${ }^{153}$,

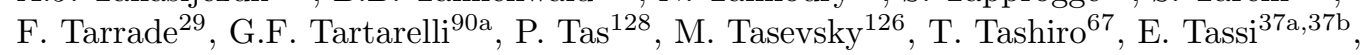
A. Tavares Delgado ${ }^{125 a, 125 b}$, Y. Tayalati ${ }^{136 d}$, F.E. Taylor ${ }^{93}$, G.N. Taylor ${ }^{87}$, W. Taylor ${ }^{160 b}$, F.A. Teischinger ${ }^{30}$, M. Teixeira Dias Castanheira ${ }^{75}$, P. Teixeira-Dias ${ }^{76}$, K.K. Temming ${ }^{48}$, H. Ten Kate ${ }^{30}$, P.K. Teng ${ }^{152}$, J.J. Teoh ${ }^{117}$, S. Terada ${ }^{65}$, K. Terashi ${ }^{156}$, J. Terron ${ }^{81}$, S. Terzo ${ }^{100}$, M. Testa ${ }^{47}$, R.J. Teuscher ${ }^{159, i}$, J. Therhaag ${ }^{21}$, T. Theveneaux-Pelzer ${ }^{34}$, J.P. Thomas ${ }^{18}$, J. Thomas-Wilsker ${ }^{76}$, E.N. Thompson ${ }^{35}$, P.D. Thompson ${ }^{18}$, P.D. Thompson ${ }^{159}$, A.S. Thompson ${ }^{53}$,

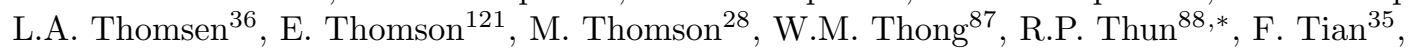
M.J. Tibbetts ${ }^{15}$, V.O. Tikhomirov ${ }^{95}, a g$, Yu.A. Tikhonov ${ }^{108, t}$, S. Timoshenko ${ }^{97}$, E. Tiouchichine ${ }^{84}$, P. Tipton ${ }^{177}$, S. Tisserant ${ }^{84}$, T. Todorov ${ }^{5}$, S. Todorova-Nova ${ }^{128}$, B. Toggerson ${ }^{7}$, J. Tojo ${ }^{69}$,

S. Tokár ${ }^{145 a}$, K. Tokushuku ${ }^{65}$, K. Tollefson ${ }^{89}$, L. Tomlinson ${ }^{83}$, M. Tomoto ${ }^{102}$, L. Tompkins ${ }^{31}$, K. Toms ${ }^{104}$, N.D. Topilin ${ }^{64}$, E. Torrence ${ }^{115}$, H. Torres ${ }^{143}$, E. Torró Pastor ${ }^{168}$, J. Toth ${ }^{84, a h}$,

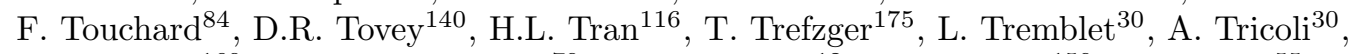
I.M. Trigger ${ }^{160 a}$, S. Trincaz-Duvoid ${ }^{79}$, M.F. Tripiana ${ }^{12}$, W. Trischuk ${ }^{159}$, B. Trocmé ${ }^{55}$,

C. Troncon ${ }^{90 a}$, M. Trottier-McDonald ${ }^{143}$, M. Trovatelli1 ${ }^{135 a, 135 b}$, P. True $^{89}$, M. Trzebinski ${ }^{39}$, A. Trzupek ${ }^{39}$, C. Tsarouchas ${ }^{30}$, J.C-L. Tseng ${ }^{119}$, P.V. Tsiareshka ${ }^{91}$, D. Tsionou ${ }^{137}$,

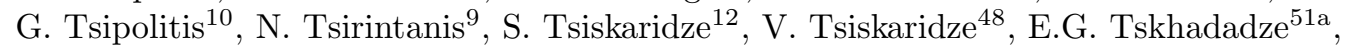

I.I. Tsukerman ${ }^{96}$, V. Tsulaia ${ }^{15}$, S. Tsuno ${ }^{65}$, D. Tsybychev ${ }^{149}$, A. Tudorache ${ }^{26 a}$, V. Tudorache ${ }^{26 a}$, A.N. Tuna ${ }^{121}$, S.A. Tupputi ${ }^{20 a, 20 b}$, S. Turchikhin ${ }^{98, a f}$, D. Turecek ${ }^{127}$, I. Turk Cakir ${ }^{4 d}$,

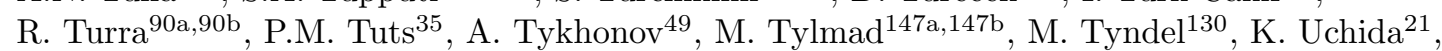

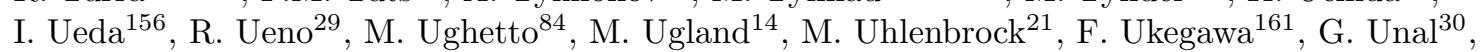
A. Undrus ${ }^{25}$, G. Unel ${ }^{164}$, F.C. Ungaro ${ }^{48}$, Y. Unno ${ }^{65}$, D. Urbaniec ${ }^{35}$, P. Urquijo ${ }^{87}$, G. Usai ${ }^{8}$, A. Usanova ${ }^{61}$, L. Vacavant ${ }^{84}$, V. Vacek ${ }^{127}$, B. Vachon ${ }^{86}$, N. Valencic ${ }^{106}$, S. Valentinetti ${ }^{20 a, 20 b}$, A. Valero ${ }^{168}$, L. Valery ${ }^{34}$, S. Valkar ${ }^{128}$, E. Valladolid Gallego ${ }^{168}$, S. Vallecorsa ${ }^{49}$, J.A. Valls Ferrer ${ }^{168}$, W. Van Den Wollenberg ${ }^{106}$, P.C. Van Der Deijl ${ }^{106}$, R. van der Geer ${ }^{106}$, H. van der Graaf ${ }^{106}$, R. Van Der Leeuw ${ }^{106}$, D. van der Ster $^{30}$, N. van Eldik ${ }^{30}$, P. van Gemmeren ${ }^{6}$, J. Van Nieuwkoop ${ }^{143}$, I. van Vulpen ${ }^{106}$, M.C. van Woerden ${ }^{30}$, M. Vanadia ${ }^{133 a, 133 b}$, W. Vandelli ${ }^{30}$, R. Vanguri ${ }^{121}$, A. Vaniachine ${ }^{6}$, P. Vankov $^{42}$, F. Vannucci ${ }^{79}$, G. Vardanyan $^{178}$, R. Vari ${ }^{133 a}$,

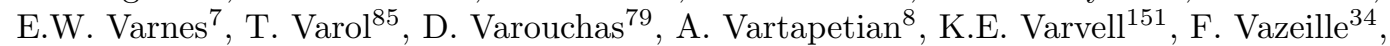
T. Vazquez Schroeder ${ }^{54}$, J. Veatch $^{7}$, F. Veloso ${ }^{125 a, 125 c}$, S. Veneziano ${ }^{133 a}$, A. Ventura ${ }^{72 a, 72 b}$, D. Ventura ${ }^{85}$, M. Venturi ${ }^{170}$, N. Venturi ${ }^{159}$, A. Venturini ${ }^{23}$, V. Vercesi ${ }^{120 a}$, M. Verducci $^{133 a, 133 b}$, W. Verkerke ${ }^{106}$, J.C. Vermeulen ${ }^{106}$, A. Vest ${ }^{44}$, M.C. Vetterli ${ }^{143, d}$, O. Viazlo ${ }^{80}$, I. Vichou ${ }^{166}$, T. Vickey ${ }^{146 c, a i}$, O.E. Vickey Boeriu ${ }^{146 c}$, G.H.A. Viehhauser ${ }^{119}$, S. Viel $^{169}$, R. Vigne ${ }^{30}$, M. Villa ${ }^{20 a, 20 b}$, M. Villaplana Perez ${ }^{90 a, 90 b}$, E. Vilucchi ${ }^{47}$, M.G. Vincter ${ }^{29}$, V.B. Vinogradov ${ }^{64}$, J. Virzi ${ }^{15}$, I. Vivarelli ${ }^{150}$, F. Vives $\operatorname{Vaque}^{3}$, S. Vlachos ${ }^{10}$, D. Vladoiu ${ }^{99}$, M. Vlasak ${ }^{127}$, A. Vogel ${ }^{21}$, M. Vogel ${ }^{32 a}$, P. Vokac ${ }^{127}$, G. Volpi ${ }^{123 a, 123 b}$, M. Volpi ${ }^{87}$, H. von der $\operatorname{Schmitt}^{100}$,

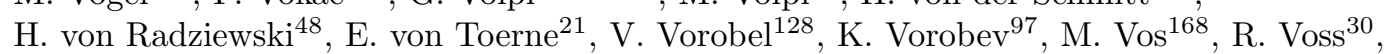
J.H. Vossebeld ${ }^{73}$, N. Vranjes ${ }^{137}$, M. Vranjes Milosavljevic ${ }^{106}$, V. Vrba ${ }^{126}$, M. Vreeswijk ${ }^{106}$, 
T. Vu Anh ${ }^{48}$, R. Vuillermet ${ }^{30}$, I. Vukotic ${ }^{31}$, Z. Vykydal ${ }^{127}$, P. Wagner ${ }^{21}$, W. Wagner ${ }^{176}$, H. Wahlberg ${ }^{70}$, S. Wahrmund ${ }^{44}$, J. Wakabayashi ${ }^{102}$, J. Walder ${ }^{71}$, R. Walker ${ }^{99}$, W. Walkowiak ${ }^{142}$, R. Wall ${ }^{177}$, P. Waller ${ }^{73}$, B. Walsh ${ }^{177}$, C. Wang ${ }^{152, a j}$, C. Wang ${ }^{45}$, F. Wang ${ }^{174}$, H. Wang $^{15}$, H. Wang ${ }^{40}$, J. Wang ${ }^{42}$, J. Wang ${ }^{33 a}$, K. Wang ${ }^{86}$, R. Wang ${ }^{104}$, S.M. Wang ${ }^{152}$, T. Wang ${ }^{21}$, X. Wang ${ }^{177}$, C. Wanotayaroj ${ }^{115}$, A. Warburton ${ }^{86}$, C.P. Ward $^{28}$, D.R. Wardrope ${ }^{77}$,

M. Warsinsky ${ }^{48}$, A. Washbrook ${ }^{46}$, C. Wasicki ${ }^{42}$, P.M. Watkins ${ }^{18}$, A.T. Watson ${ }^{18}$, I.J. Watson ${ }^{151}$, M.F. Watson ${ }^{18}$, G. Watts ${ }^{139}$, S. Watts ${ }^{83}$, B.M. Waugh ${ }^{77}$, S. Webb ${ }^{83}$, M.S. Weber ${ }^{17}$,

S.W. Weber ${ }^{175}$, J.S. Webster ${ }^{31}$, A.R. Weidberg ${ }^{119}$, P. Weigell ${ }^{100}$, B. Weinert ${ }^{60}$, J. Weingarten ${ }^{54}$, C. Weiser ${ }^{48}$, H. Weits ${ }^{106}$, P.S. Wells ${ }^{30}$, T. Wenaus ${ }^{25}$, D. Wendland ${ }^{16}$, Z. Weng ${ }^{152, a e}$, T. Wengler ${ }^{30}$,

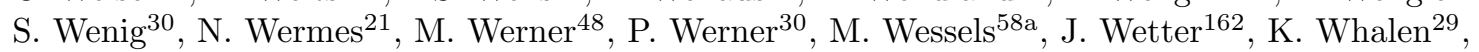
A. White ${ }^{8}$, M.J. White ${ }^{1}$, R. White ${ }^{32 b}$, S. White ${ }^{123 a, 123 b}$, D. Whiteson ${ }^{164}$, D. Wicke ${ }^{176}$, F.J. Wickens ${ }^{130}$, W. Wiedenmann ${ }^{174}$, M. Wielers ${ }^{130}$, P. Wienemann ${ }^{21}$, C. Wiglesworth ${ }^{36}$, L.A.M. Wiik-Fuchs ${ }^{21}$, P.A. Wijeratne ${ }^{77}$, A. Wildauer ${ }^{100}$, M.A. Wildt ${ }^{42, a k}$, H.G. Wilkens ${ }^{30}$, J.Z. Will ${ }^{99}$, H.H. Williams ${ }^{121}$, S. Williams ${ }^{28}$, C. Willis ${ }^{89}$, S. Willocq ${ }^{85}$, A. Wilson ${ }^{88}$, J.A. Wilson ${ }^{18}$, I. Wingerter-Seez ${ }^{5}$, F. Winklmeier ${ }^{115}$, B.T. Winter ${ }^{21}$, M. Wittgen ${ }^{144}$, T. Wittig ${ }^{43}$, J. Wittkowski ${ }^{99}$, S.J. Wollstadt ${ }^{82}$, M.W. Wolter ${ }^{39}$, H. Wolters ${ }^{125 a, 125 c}$, B.K. Wosiek ${ }^{39}$,

J. Wotschack ${ }^{30}$, M.J. Woudstra ${ }^{83}$, K.W. Wozniak ${ }^{39}$, M. Wright ${ }^{53}$, M. Wu ${ }^{55}$, S.L. Wu ${ }^{174}, \mathrm{X} \mathrm{Wu}^{49}$, Y. Wu ${ }^{88}$, E. Wulf ${ }^{35}$, T.R. Wyatt ${ }^{83}$, B.M. Wynne ${ }^{46}$, S. Xella ${ }^{36}$, M. Xiao ${ }^{137}$, D. Xu ${ }^{33 a}$, L. Xu ${ }^{33 b, a l}$, B. Yabsley ${ }^{151}$, S. Yacoob ${ }^{146 b, a m}$, R. Yakabe ${ }^{66}$, M. Yamada ${ }^{65}$, H. Yamaguchi ${ }^{156}$, Y. Yamaguchi ${ }^{117}$,

A. Yamamoto ${ }^{65}$, K. Yamamoto ${ }^{63}$, S. Yamamoto ${ }^{156}$, T. Yamamura ${ }^{156}$, T. Yamanaka ${ }^{156}$, K. Yamauchi ${ }^{102}$, Y. Yamazaki ${ }^{66}$, Z. Yan ${ }^{22}$, H. Yang ${ }^{33 e}$, H. Yang ${ }^{174}$, U.K. Yang ${ }^{83}$, Y. Yang ${ }^{110}$,

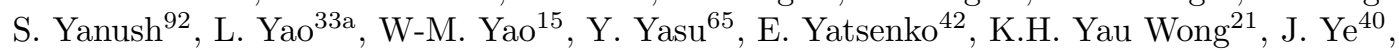

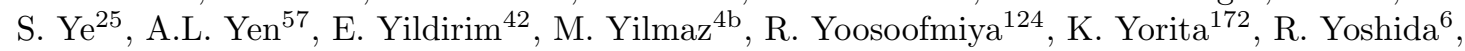
K. Yoshihara ${ }^{156}$, C. Young ${ }^{144}$, C.J.S. Young ${ }^{30}$, S. Youssef ${ }^{22}$, D.R. Yu ${ }^{15}$, J. Yu $^{8}$, J.M. Yu ${ }^{88}$, J. Yu ${ }^{113}$, L. Yuan ${ }^{66}$, A. Yurkewicz ${ }^{107}$, I. Yusuff ${ }^{28, a n}$, B. Zabinski ${ }^{39}$, R. Zaidan ${ }^{62}$,

A.M. Zaitsev ${ }^{129, a a}$, A. Zaman ${ }^{149}$, S. Zambito ${ }^{23}$, L. Zanello ${ }^{133 a, 133 b}$, D. Zanzi ${ }^{100}$, C. Zeitnitz ${ }^{176}$, M. Zeman ${ }^{127}$, A. Zemla ${ }^{38 a}$, K. Zengel ${ }^{23}$, O. Zenin ${ }^{129}$, T. Ženišs ${ }^{145 a}$, D. Zerwas ${ }^{116}$,

G. Zevi della Porta ${ }^{57}$, D. Zhang ${ }^{88}$, F. Zhang ${ }^{174}$, H. Zhang ${ }^{89}$, J. Zhang ${ }^{6}$, L. Zhang ${ }^{152}$, X. Zhang ${ }^{33 d}$, Z. Zhang ${ }^{116}$, Z. Zhao ${ }^{33 \mathrm{~b}}$, A. Zhemchugov ${ }^{64}$, J. Zhong ${ }^{119}$, B. Zhou ${ }^{88}$, L. Zhou ${ }^{35}$, N. Zhou ${ }^{164}$, C.G. Zhu ${ }^{33 d}$, H. Zhu ${ }^{33 a}$, J. Zhu ${ }^{88}$, Y. Zhu ${ }^{33 b}$, X. Zhuang ${ }^{33 a}$, K. Zhukov ${ }^{95}$, A. Zibell ${ }^{175}$,

D. Zieminska ${ }^{60}$, N.I. Zimine ${ }^{64}$, C. Zimmermann ${ }^{82}$, R. Zimmermann ${ }^{21}$, S. Zimmermannn ${ }^{21}$,

S. Zimmermann ${ }^{48}$, Z. Zinonos ${ }^{54}$, M. Ziolkowski ${ }^{142}$, G. Zobernig ${ }^{174}$, A. Zoccoli ${ }^{20 a, 20 b}$,

M. zur Nedden ${ }^{16}$, G. Zurzolo ${ }^{103 a}, 103 \mathrm{~b}$, V. Zutshi ${ }^{107}$, L. Zwalinski $^{30}$

1 Department of Physics, University of Adelaide, Adelaide, Australia

2 Physics Department, SUNY Albany, Albany NY, United States of America

3 Department of Physics, University of Alberta, Edmonton AB, Canada

4 (a) Department of Physics, Ankara University, Ankara; ${ }^{(b)}$ Department of Physics, Gazi University, Ankara; ${ }^{(c)}$ Division of Physics, TOBB University of Economics and Technology, Ankara; ${ }^{(d)}$ Turkish Atomic Energy Authority, Ankara, Turkey

5 LAPP, CNRS/IN2P3 and Université de Savoie, Annecy-le-Vieux, France

6 High Energy Physics Division, Argonne National Laboratory, Argonne IL, United States of America

7 Department of Physics, University of Arizona, Tucson AZ, United States of America

8 Department of Physics, The University of Texas at Arlington, Arlington TX, United States of America

9 Physics Department, University of Athens, Athens, Greece

10 Physics Department, National Technical University of Athens, Zografou, Greece

11 Institute of Physics, Azerbaijan Academy of Sciences, Baku, Azerbaijan

12 Institut de Física d'Altes Energies and Departament de Física de la Universitat Autònoma de Barcelona, Barcelona, Spain

13 (a) Institute of Physics, University of Belgrade, Belgrade; ${ }^{(b)}$ Vinca Institute of Nuclear Sciences, University of Belgrade, Belgrade, Serbia

14 Department for Physics and Technology, University of Bergen, Bergen, Norway 
15 Physics Division, Lawrence Berkeley National Laboratory and University of California, Berkeley CA, United States of America

16 Department of Physics, Humboldt University, Berlin, Germany

17 Albert Einstein Center for Fundamental Physics and Laboratory for High Energy Physics, University of Bern, Bern, Switzerland

18 School of Physics and Astronomy, University of Birmingham, Birmingham, United Kingdom

19 (a) Department of Physics, Bogazici University, Istanbul; ${ }^{(b)}$ Department of Physics, Dogus University, Istanbul; ${ }^{(c)}$ Department of Physics Engineering, Gaziantep University, Gaziantep, Turkey

20 (a) INFN Sezione di Bologna; ${ }^{(b)}$ Dipartimento di Fisica e Astronomia, Università di Bologna, Bologna, Italy

21 Physikalisches Institut, University of Bonn, Bonn, Germany

22 Department of Physics, Boston University, Boston MA, United States of America

23 Department of Physics, Brandeis University, Waltham MA, United States of America

24 (a) Universidade Federal do Rio De Janeiro COPPE/EE/IF, Rio de Janeiro; ${ }^{(b)}$ Federal University of Juiz de Fora (UFJF), Juiz de Fora; ${ }^{(c)}$ Federal University of Sao Joao del Rei (UFSJ), Sao Joao del Rei; ${ }^{(d)}$ Instituto de Fisica, Universidade de Sao Paulo, Sao Paulo, Brazil

25 Physics Department, Brookhaven National Laboratory, Upton NY, United States of America

26 (a) National Institute of Physics and Nuclear Engineering, Bucharest; ${ }^{(b)}$ National Institute for Research and Development of Isotopic and Molecular Technologies, Physics Department, Cluj Napoca; ${ }^{(c)}$ University Politehnica Bucharest, Bucharest; ${ }^{(d)}$ West University in Timisoara, Timisoara, Romania

27 Departamento de Física, Universidad de Buenos Aires, Buenos Aires, Argentina

28 Cavendish Laboratory, University of Cambridge, Cambridge, United Kingdom

29 Department of Physics, Carleton University, Ottawa ON, Canada

30 CERN, Geneva, Switzerland

31 Enrico Fermi Institute, University of Chicago, Chicago IL, United States of America

32 (a) Departamento de Física, Pontificia Universidad Católica de Chile, Santiago; ${ }^{(b)}$ Departamento de Física, Universidad Técnica Federico Santa María, Valparaíso, Chile

33 (a) Institute of High Energy Physics, Chinese Academy of Sciences, Beijing; ${ }^{(b)}$ Department of Modern Physics, University of Science and Technology of China, Anhui; ${ }^{(c)}$ Department of Physics, Nanjing University, Jiangsu; ${ }^{(d)}$ School of Physics, Shandong University, Shandong; ${ }^{(e)}$ Physics Department, Shanghai Jiao Tong University, Shanghai, China

34 Laboratoire de Physique Corpusculaire, Clermont Université and Université Blaise Pascal and CNRS/IN2P3, Clermont-Ferrand, France

35 Nevis Laboratory, Columbia University, Irvington NY, United States of America

36 Niels Bohr Institute, University of Copenhagen, Kobenhavn, Denmark

37 (a) INFN Gruppo Collegato di Cosenza, Laboratori Nazionali di Frascati; ${ }^{(b)}$ Dipartimento di Fisica, Università della Calabria, Rende, Italy

38 (a) AGH University of Science and Technology, Faculty of Physics and Applied Computer Science, Krakow; ${ }^{(b)}$ Marian Smoluchowski Institute of Physics, Jagiellonian University, Krakow, Poland

39 The Henryk Niewodniczanski Institute of Nuclear Physics, Polish Academy of Sciences, Krakow, Poland

40 Physics Department, Southern Methodist University, Dallas TX, United States of America

41 Physics Department, University of Texas at Dallas, Richardson TX, United States of America

42 DESY, Hamburg and Zeuthen, Germany

43 Institut für Experimentelle Physik IV, Technische Universität Dortmund, Dortmund, Germany

44 Institut für Kern- und Teilchenphysik, Technische Universität Dresden, Dresden, Germany

45 Department of Physics, Duke University, Durham NC, United States of America

46 SUPA - School of Physics and Astronomy, University of Edinburgh, Edinburgh, United Kingdom

47 INFN Laboratori Nazionali di Frascati, Frascati, Italy

48 Fakultät für Mathematik und Physik, Albert-Ludwigs-Universität, Freiburg, Germany 
49 Section de Physique, Université de Genève, Geneva, Switzerland

50 (a) INFN Sezione di Genova; ${ }^{(b)}$ Dipartimento di Fisica, Università di Genova, Genova, Italy

$51{ }^{(a)}$ E. Andronikashvili Institute of Physics, Iv. Javakhishvili Tbilisi State University, Tbilisi; ${ }^{(b)}$ High Energy Physics Institute, Tbilisi State University, Tbilisi, Georgia

52 II Physikalisches Institut, Justus-Liebig-Universität Giessen, Giessen, Germany

53 SUPA - School of Physics and Astronomy, University of Glasgow, Glasgow, United Kingdom

54 II Physikalisches Institut, Georg-August-Universität, Göttingen, Germany

55 Laboratoire de Physique Subatomique et de Cosmologie, Université Grenoble-Alpes, CNRS/IN2P3, Grenoble, France

56 Department of Physics, Hampton University, Hampton VA, United States of America

57 Laboratory for Particle Physics and Cosmology, Harvard University, Cambridge MA, United States of America

58 (a) Kirchhoff-Institut für Physik, Ruprecht-Karls-Universität Heidelberg, Heidelberg; ${ }^{(b)}$ Physikalisches Institut, Ruprecht-Karls-Universität Heidelberg, Heidelberg; ${ }^{(c)}$ ZITI Institut für technische Informatik, Ruprecht-Karls-Universität Heidelberg, Mannheim, Germany

59 Faculty of Applied Information Science, Hiroshima Institute of Technology, Hiroshima, Japan

60 Department of Physics, Indiana University, Bloomington IN, United States of America

61 Institut für Astro- und Teilchenphysik, Leopold-Franzens-Universität, Innsbruck, Austria

62 University of Iowa, Iowa City IA, United States of America

63 Department of Physics and Astronomy, Iowa State University, Ames IA, United States of America

64 Joint Institute for Nuclear Research, JINR Dubna, Dubna, Russia

65 KEK, High Energy Accelerator Research Organization, Tsukuba, Japan

66 Graduate School of Science, Kobe University, Kobe, Japan

67 Faculty of Science, Kyoto University, Kyoto, Japan

68 Kyoto University of Education, Kyoto, Japan

69 Department of Physics, Kyushu University, Fukuoka, Japan

70 Instituto de Física La Plata, Universidad Nacional de La Plata and CONICET, La Plata, Argentina

71 Physics Department, Lancaster University, Lancaster, United Kingdom

72 (a) INFN Sezione di Lecce; ${ }^{(b)}$ Dipartimento di Matematica e Fisica, Università del Salento, Lecce, Italy

73 Oliver Lodge Laboratory, University of Liverpool, Liverpool, United Kingdom

74 Department of Physics, Jožef Stefan Institute and University of Ljubljana, Ljubljana, Slovenia

75 School of Physics and Astronomy, Queen Mary University of London, London, United Kingdom

76 Department of Physics, Royal Holloway University of London, Surrey, United Kingdom

77 Department of Physics and Astronomy, University College London, London, United Kingdom

78 Louisiana Tech University, Ruston LA, United States of America

79 Laboratoire de Physique Nucléaire et de Hautes Energies, UPMC and Université Paris-Diderot and CNRS/IN2P3, Paris, France

80 Fysiska institutionen, Lunds universitet, Lund, Sweden

81 Departamento de Fisica Teorica C-15, Universidad Autonoma de Madrid, Madrid, Spain

82 Institut für Physik, Universität Mainz, Mainz, Germany

83 School of Physics and Astronomy, University of Manchester, Manchester, United Kingdom

84 CPPM, Aix-Marseille Université and CNRS/IN2P3, Marseille, France

85 Department of Physics, University of Massachusetts, Amherst MA, United States of America

86 Department of Physics, McGill University, Montreal QC, Canada

87 School of Physics, University of Melbourne, Victoria, Australia

88 Department of Physics, The University of Michigan, Ann Arbor MI, United States of America

89 Department of Physics and Astronomy, Michigan State University, East Lansing MI, United States of America

$90 \quad{ }^{(a)}$ INFN Sezione di Milano; ${ }^{(b)}$ Dipartimento di Fisica, Università di Milano, Milano, Italy

91 B.I. Stepanov Institute of Physics, National Academy of Sciences of Belarus, Minsk, Republic of Belarus 
National Scientific and Educational Centre for Particle and High Energy Physics, Minsk, Republic of Belarus

93 Department of Physics, Massachusetts Institute of Technology, Cambridge MA, United States of America

94 Group of Particle Physics, University of Montreal, Montreal QC, Canada

95 P.N. Lebedev Institute of Physics, Academy of Sciences, Moscow, Russia

96 Institute for Theoretical and Experimental Physics (ITEP), Moscow, Russia

97 Moscow Engineering and Physics Institute (MEPhI), Moscow, Russia

98 D.V.Skobeltsyn Institute of Nuclear Physics, M.V.Lomonosov Moscow State University, Moscow, Russia

99 Fakultät für Physik, Ludwig-Maximilians-Universität München, München, Germany

100 Max-Planck-Institut für Physik (Werner-Heisenberg-Institut), München, Germany

101 Nagasaki Institute of Applied Science, Nagasaki, Japan

102 Graduate School of Science and Kobayashi-Maskawa Institute, Nagoya University, Nagoya, Japan

103 (a) INFN Sezione di Napoli; ${ }^{(b)}$ Dipartimento di Fisica, Università di Napoli, Napoli, Italy

104 Department of Physics and Astronomy, University of New Mexico, Albuquerque NM, United States of America

105 Institute for Mathematics, Astrophysics and Particle Physics, Radboud University Nijmegen/Nikhef, Nijmegen, Netherlands

106 Nikhef National Institute for Subatomic Physics and University of Amsterdam, Amsterdam, Netherlands

107 Department of Physics, Northern Illinois University, DeKalb IL, United States of America

108 Budker Institute of Nuclear Physics, SB RAS, Novosibirsk, Russia

109 Department of Physics, New York University, New York NY, United States of America

110 Ohio State University, Columbus OH, United States of America

111 Faculty of Science, Okayama University, Okayama, Japan

112 Homer L. Dodge Department of Physics and Astronomy, University of Oklahoma, Norman OK, United States of America

113 Department of Physics, Oklahoma State University, Stillwater OK, United States of America

114 Palacký University, RCPTM, Olomouc, Czech Republic

115 Center for High Energy Physics, University of Oregon, Eugene OR, United States of America

116 LAL, Université Paris-Sud and CNRS/IN2P3, Orsay, France

117 Graduate School of Science, Osaka University, Osaka, Japan

118 Department of Physics, University of Oslo, Oslo, Norway

119 Department of Physics, Oxford University, Oxford, United Kingdom

120 (a) INFN Sezione di Pavia; ${ }^{(b)}$ Dipartimento di Fisica, Università di Pavia, Pavia, Italy

121 Department of Physics, University of Pennsylvania, Philadelphia PA, United States of America

122 Petersburg Nuclear Physics Institute, Gatchina, Russia

123 (a) INFN Sezione di Pisa; ${ }^{(b)}$ Dipartimento di Fisica E. Fermi, Università di Pisa, Pisa, Italy

124 Department of Physics and Astronomy, University of Pittsburgh, Pittsburgh PA, United States of America

125 (a) Laboratorio de Instrumentacao e Fisica Experimental de Particulas - LIP, Lisboa; ${ }^{(b)}$ Faculdade de Ciências, Universidade de Lisboa, Lisboa; ${ }^{(c)}$ Department of Physics, University of Coimbra, Coimbra; ${ }^{(d)}$ Centro de Física Nuclear da Universidade de Lisboa, Lisboa; ${ }^{(e)}$ Departamento de Fisica, Universidade do Minho, Braga; ${ }^{(f)}$ Departamento de Fisica Teorica y del Cosmos and CAFPE, Universidad de Granada, Granada (Spain); ${ }^{(g)}$ Dep Fisica and CEFITEC of Faculdade de Ciencias e Tecnologia, Universidade Nova de Lisboa, Caparica, Portugal

126 Institute of Physics, Academy of Sciences of the Czech Republic, Praha, Czech Republic

127 Czech Technical University in Prague, Praha, Czech Republic

128 Faculty of Mathematics and Physics, Charles University in Prague, Praha, Czech Republic

129 State Research Center Institute for High Energy Physics, Protvino, Russia

130 Particle Physics Department, Rutherford Appleton Laboratory, Didcot, United Kingdom 
131 Physics Department, University of Regina, Regina SK, Canada

132 Ritsumeikan University, Kusatsu, Shiga, Japan

133 (a) INFN Sezione di Roma; ${ }^{(b)}$ Dipartimento di Fisica, Sapienza Università di Roma, Roma, Italy

134 (a) INFN Sezione di Roma Tor Vergata; ${ }^{(b)}$ Dipartimento di Fisica, Università di Roma Tor Vergata, Roma, Italy

135 (a) INFN Sezione di Roma Tre; ${ }^{(b)}$ Dipartimento di Matematica e Fisica, Università Roma Tre, Roma, Italy

136 (a) Faculté des Sciences Ain Chock, Réseau Universitaire de Physique des Hautes Energies Université Hassan II, Casablanca; ${ }^{(b)}$ Centre National de l'Energie des Sciences Techniques Nucleaires, Rabat; ${ }^{(c)}$ Faculté des Sciences Semlalia, Université Cadi Ayyad, LPHEA-Marrakech; ${ }^{(d)}$ Faculté des Sciences, Université Mohamed Premier and LPTPM, Oujda; ${ }^{(e)}$ Faculté des sciences, Université Mohammed V-Agdal, Rabat, Morocco

137 DSM/IRFU (Institut de Recherches sur les Lois Fondamentales de l'Univers), CEA Saclay (Commissariat à l'Energie Atomique et aux Energies Alternatives), Gif-sur-Yvette, France

138 Santa Cruz Institute for Particle Physics, University of California Santa Cruz, Santa Cruz CA, United States of America

139 Department of Physics, University of Washington, Seattle WA, United States of America

140 Department of Physics and Astronomy, University of Sheffield, Sheffield, United Kingdom

141 Department of Physics, Shinshu University, Nagano, Japan

142 Fachbereich Physik, Universität Siegen, Siegen, Germany

143 Department of Physics, Simon Fraser University, Burnaby BC, Canada

144 SLAC National Accelerator Laboratory, Stanford CA, United States of America

145 (a) Faculty of Mathematics, Physics \& Informatics, Comenius University, Bratislava; ${ }^{(b)}$

Department of Subnuclear Physics, Institute of Experimental Physics of the Slovak Academy of Sciences, Kosice, Slovak Republic

146 (a) Department of Physics, University of Cape Town, Cape Town; ${ }^{(b)}$ Department of Physics, University of Johannesburg, Johannesburg; ${ }^{(c)}$ School of Physics, University of the Witwatersrand, Johannesburg, South Africa

147 (a) Department of Physics, Stockholm University; ${ }^{(b)}$ The Oskar Klein Centre, Stockholm, Sweden

148 Physics Department, Royal Institute of Technology, Stockholm, Sweden

149 Departments of Physics \& Astronomy and Chemistry, Stony Brook University, Stony Brook NY, United States of America

150 Department of Physics and Astronomy, University of Sussex, Brighton, United Kingdom

151 School of Physics, University of Sydney, Sydney, Australia

152 Institute of Physics, Academia Sinica, Taipei, Taiwan

153 Department of Physics, Technion: Israel Institute of Technology, Haifa, Israel

154 Raymond and Beverly Sackler School of Physics and Astronomy, Tel Aviv University, Tel Aviv, Israel

155 Department of Physics, Aristotle University of Thessaloniki, Thessaloniki, Greece

156 International Center for Elementary Particle Physics and Department of Physics, The University of Tokyo, Tokyo, Japan

157 Graduate School of Science and Technology, Tokyo Metropolitan University, Tokyo, Japan

158 Department of Physics, Tokyo Institute of Technology, Tokyo, Japan

159 Department of Physics, University of Toronto, Toronto ON, Canada

160 (a) TRIUMF, Vancouver BC; ${ }^{(b)}$ Department of Physics and Astronomy, York University, Toronto ON, Canada

161 Faculty of Pure and Applied Sciences, University of Tsukuba, Tsukuba, Japan

162 Department of Physics and Astronomy, Tufts University, Medford MA, United States of America

163 Centro de Investigaciones, Universidad Antonio Narino, Bogota, Colombia

164 Department of Physics and Astronomy, University of California Irvine, Irvine CA, United States of America

165 (a) INFN Gruppo Collegato di Udine, Sezione di Trieste, Udine; ${ }^{(b)}$ ICTP, Trieste; ${ }^{(c)}$ Dipartimento di Chimica, Fisica e Ambiente, Università di Udine, Udine, Italy 
166 Department of Physics, University of Illinois, Urbana IL, United States of America

167 Department of Physics and Astronomy, University of Uppsala, Uppsala, Sweden

168 Instituto de Física Corpuscular (IFIC) and Departamento de Física Atómica, Molecular y Nuclear and Departamento de Ingeniería Electrónica and Instituto de Microelectrónica de Barcelona (IMB-CNM), University of Valencia and CSIC, Valencia, Spain

169 Department of Physics, University of British Columbia, Vancouver BC, Canada

170 Department of Physics and Astronomy, University of Victoria, Victoria BC, Canada

171 Department of Physics, University of Warwick, Coventry, United Kingdom

172 Waseda University, Tokyo, Japan

173 Department of Particle Physics, The Weizmann Institute of Science, Rehovot, Israel

174 Department of Physics, University of Wisconsin, Madison WI, United States of America

175 Fakultät für Physik und Astronomie, Julius-Maximilians-Universität, Würzburg, Germany

176 Fachbereich C Physik, Bergische Universität Wuppertal, Wuppertal, Germany

177 Department of Physics, Yale University, New Haven CT, United States of America

178 Yerevan Physics Institute, Yerevan, Armenia

179 Centre de Calcul de l'Institut National de Physique Nucléaire et de Physique des Particules (IN2P3), Villeurbanne, France

a Also at Department of Physics, King's College London, London, United Kingdom

${ }^{b}$ Also at Institute of Physics, Azerbaijan Academy of Sciences, Baku, Azerbaijan

c Also at Particle Physics Department, Rutherford Appleton Laboratory, Didcot, United Kingdom

$d$ Also at TRIUMF, Vancouver BC, Canada

e Also at Department of Physics, California State University, Fresno CA, United States of America

$f$ Also at Tomsk State University, Tomsk, Russia

$g$ Also at CPPM, Aix-Marseille Université and CNRS/IN2P3, Marseille, France

$h$ Also at Università di Napoli Parthenope, Napoli, Italy

$i$ Also at Institute of Particle Physics (IPP), Canada

$j$ Also at Department of Physics, St. Petersburg State Polytechnical University, St. Petersburg, Russia

$k$ Also at Chinese University of Hong Kong, China

$l$ Also at Department of Financial and Management Engineering, University of the Aegean, Chios, Greece

$m$ Also at Louisiana Tech University, Ruston LA, United States of America

$n$ Also at Institucio Catalana de Recerca i Estudis Avancats, ICREA, Barcelona, Spain

o Also at Department of Physics, The University of Texas at Austin, Austin TX, United States of America

$p$ Also at Institute of Theoretical Physics, Ilia State University, Tbilisi, Georgia

$q$ Also at CERN, Geneva, Switzerland

$r$ Also at Ochadai Academic Production, Ochanomizu University, Tokyo, Japan

$s$ Also at Manhattan College, New York NY, United States of America

${ }^{t}$ Also at Novosibirsk State University, Novosibirsk, Russia

u Also at Institute of Physics, Academia Sinica, Taipei, Taiwan

$v$ Also at LAL, Université Paris-Sud and CNRS/IN2P3, Orsay, France

$w$ Also at Academia Sinica Grid Computing, Institute of Physics, Academia Sinica, Taipei, Taiwan

$x$ Also at Laboratoire de Physique Nucléaire et de Hautes Energies, UPMC and Université Paris-Diderot and CNRS/IN2P3, Paris, France

$y$ Also at School of Physical Sciences, National Institute of Science Education and Research, Bhubaneswar, India

$z$ Also at Dipartimento di Fisica, Sapienza Università di Roma, Roma, Italy

aa Also at Moscow Institute of Physics and Technology State University, Dolgoprudny, Russia

$a b$ Also at Section de Physique, Université de Genève, Geneva, Switzerland

ac Also at International School for Advanced Studies (SISSA), Trieste, Italy 
ad Also at Department of Physics and Astronomy, University of South Carolina, Columbia SC, United States of America

ae Also at School of Physics and Engineering, Sun Yat-sen University, Guangzhou, China

af Also at Faculty of Physics, M.V.Lomonosov Moscow State University, Moscow, Russia

ag Also at Moscow Engineering and Physics Institute (MEPhI), Moscow, Russia

ah Also at Institute for Particle and Nuclear Physics, Wigner Research Centre for Physics, Budapest, Hungary

ai Also at Department of Physics, Oxford University, Oxford, United Kingdom

aj Also at Department of Physics, Nanjing University, Jiangsu, China

ak Also at Institut für Experimentalphysik, Universität Hamburg, Hamburg, Germany

al Also at Department of Physics, The University of Michigan, Ann Arbor MI, United States of America

am Also at Discipline of Physics, University of KwaZulu-Natal, Durban, South Africa

an Also at University of Malaya, Department of Physics, Kuala Lumpur, Malaysia

* Deceased 